\title{
22nd Annual SASRO Meeting
}

Congress president: Prof. Dr. Matthias Guckenberger

University of Zurich, Campus Irchel, Winterthurerstraße 190, 8057 Zürich

Scientific committees

Scientific committee-physicians

Nicolaus Andratschke

Abdelkarim Allal

Antonella Richetti

Olgun Eliçin

Fernanda Herrera

Scientific committee-medical physicists

Jan Unkelbach

Peter Manser

Raphael Möckli

Tony Lomax

Scientific committee-biologists

Martin Pruschy

Stephan Bodis

Scientific committee-technicians

Benjamin König-Nettelmann

Christopher Winter 


\section{Clinical track}

\section{The multidisciplinary approach to the management of post- mastectomy RT and breast reconstruction: The experience of the Ticino Breast Unit}

\author{
MC. Valli ${ }^{1,2}$, S. Cima ${ }^{1,2}$, M. I. Donegani ${ }^{1,2}$, A. Richetti ${ }^{1,2}$ \\ ${ }^{1}$ Radiation Oncology, Oncology Institute of Southern Switzerland, \\ Bellinzona, Switzerland \\ ${ }^{2}$ Centro di Senologia della Svizzera Italiana (CSSI), Bellinzona, \\ Switzerland
} cussion on reconstruction modalities in breast cancer pts, planned for mastectomy and adjuvant RT.

Methods: In our Breast Unit all pts are discussed primarily during a pre-operative multidisciplinary meeting for optimizing the treatment schedule. If mastectomy and adjuvant RT are required, the proper reconstruction technique is arranged by the plastic surgeon and the radiation oncologist, taking into account the patient's preference. The records of 46 pts, treated with mastectomy in 2017, have been reviewed. Results: 226 pts underwent a breast operation in 2017: $180(80 \%)$ conservative surgery and $46(20 \%)$ radical or skin/nipple sparing mastectomy. Immediate breast reconstruction has been performed in 28 pts: 19 with expander, 5 with autologous tissue and 4 with definitive prosthesis. Five of them have been treated with adjuvant RT according to stage: 4 have been reconstructed with expander (one had bilateral breast cancer) and 1 with autologous tissue. All pts, except one, have been treated on chest wall plus SC/IM chain with VMAT/RapidArc technique and respiratory gating if left or bilateral chest wall.

Conclusion: The lack of clear guidelines on the best reconstruction technique, if post-op RT is required, reinforce the duty of a pre-operative multidisciplinary discussion. Our actual policy consists in RT on completely inflated expander and definitive placement of permanent implant at least six months after the end of RT. The pre-operative discussion allowed us to avoid definitive prosthesis reconstruction in patients candidates to RT. We moreover ask for checking clinically the pts together with the plastic surgeon for a clear and shared explanation of the pros and contra of the different reconstruction modalities, if post-operative RT is prescribed. Poster

\section{Mobile health technologies for palliative care patients}

M.Pavic ${ }^{1 *}$, V.Klaas ${ }^{2 *}$, J.Kraft ${ }^{1}$, M.Guckenberger ${ }^{1}$, G.Tröster ${ }^{2}$, G.Theile ${ }^{1}$

*both authors contributed equally

${ }^{1}$ Radiation Oncology/Competence Center Palliative Care, University Hospital Zurich, Zurich, Switzerland

${ }^{2}$ Wearable Computing Laboratory, ETH, Zurich, Zurich, Switzerland

Aims: Discharge from hospital is a vulnerable phase in palliative patients' trajectories resulting in frequent unplanned readmissions. We examined the feasibility of remote monitoring of palliative patients, aiming to detect deterioration of health status early and to prevent emergency readmissions.

Methods: Patients treated with palliative intent in an inpatient setting were recruited for this prospective single-center observational study. Inclusion criteria were age $>18$, a severe medical condition (metastatic cancer or severe cardiac/pulmonary disease), ECOG $\leq 2 / \mathrm{KPS} \geq 50 \%$, no relevant cognitive impairment and good knowledge of German language. Patients were provided with wearables in form of a smartphone and a bracelet collecting vital data. These data as well as questionnaires about symptoms were collected over 12 weeks. The study was conducted in an exploratory and descriptive design.

Results: Between 02/17-02/18 71 patients were eligible, of which 31 patients between 39-85 y (median 62, SD 11.4) agreed to participate.
25/31 patients completed the whole study period, 4 died in between, 2 discontinued. Completion rate of questionnaires was $75 \%$. On average, the bracelet was worn at $53 \%$ of the days. Wearing time for these days was on average $63 \%$ (8.00-20.00). Smartphone was worn at $85 \%$ of the days and wearing time was $50 \%(08.00-20.00)$. Heart rate variability (HRV) while resting (Root Mean Square of the Successive Differences) and resting heart rate differed between groups of patients (readmission vs no readmission). Differences were nominally significant ( $p=.011, p=.036$, resp.). Due to explorative design involving multiple testing, these results have to be confirmed by an independent study.

Conclusion: Monitoring of palliative patients using wearables and smartphone technology over 12 weeks is feasible. First results indicate that resting HRV and heart rate might predict readmission. Poster

\section{Sexual function after prostate seeds brachytherapy-a long- term single center experience}

Daniel Taussky

radio-oncologie, université de Montréal, Montréal, Canada

Purpose: Prostate brachytherapy (PB) with radioactive seeds has been shown to have a favorable outcome in preserving erections. In this present study we analyze its long-term effect on erectile function (EF) and other influencing factors.

Material and methods: We included all patients treated with seed$\mathrm{PB}$ as monotherapy who were prospectively followed and EF evaluated and recorded at our center. All patients had to have recorded pretreatment $\mathrm{EF}$ and at least one post-PB evaluation. EF was graded with the Common Terminology Criteria for Adverse Events (CTCAE), Version 4.0: $0=$ no dysfunction; $1=$ decrease, but no intervention needed; $2=$ decrease, intervention indicated; $3=$ decrease but erectile intervention not helpful. Only potent patients were included (CTCAE $\leq 2$ ). Binary logistic regression analysis was used to predict factors associated with preserved $\mathrm{EF}$ after $\mathrm{PB}$, defined as having sufficient $\mathrm{EF}$ for sexual activity with or without the help of medication (CTCAE $\leq 2)$.

Results: Median age was 64 years (IQR 60-68), 12\% had diabetes, $44 \%$ hypertension, $10 \%$ a previous cardiac event. In general, of patients potent (grade $0-2$ ) at baseline from the time of the first evaluation throughout the last evaluation (8-65 months) $11-16 \%$ were unable to have intercourse (grade 3 toxicity). Of the patient who did not need any medical or mechanical help at baseline, only 10-24\% latter needed help (grade 2 dysfunction) and 9-14\% became important (grade 3). Erectile dysfunction plateaued at 26 months. On multivariate analysis diabetes (HR 3.9, 95\%CI 1.8-8.1, $p<0.001$ ) age $>65$ years (HR 2.4, $1.2-4.8, p=0.009$ ) and $\mathrm{EF}$ at baseline (HR per point increase 2.43, $1.6-6.4, p<0.001)$ remained significant, but not hypertension $(p=0.1)$. Conclusion: Preservation of EF after PB in potent patients is excellent. Only $14 \%$ lose their EF at a maximum of 65 months of follow-up. Known risk-factors for arteriosclerosis as well as age and baseline EF determine whether a patient will be able to remain sexually active after PB. Poster

\section{Systematic review \& network meta-analysis to identify the optimum nonoperative therapeutic strategies in locally advanced cancer cervix}

Prof. Dr. med. Niloy Ranjan Datta, E. Stutz, S. Gômez, S. Bodis

RadioOnkologie Zentrum KSA-KSB, Kantonsspital Aarau, Aarau, Switzerland

Aims: Treatment options in locally advanced cancer cervix (LACC) (stages IIB-IVA) have evolved around radiotherapy (RT) and/or chemotherapy (CT), hypoxic cell sensitizers (HypCS), immunotherapy (Imm) and loco-regional moderate hyperthermia (HT). A systematic review and network meta-analysis (NMA) was conducted to synthesize the clinical evidence for efficacy and safety of these approaches for 
long-term loco-regional control (LRC), overall survival (OS), grade III+ acute (AM) and late morbidity (LM).

Methods: Five major databases were searched as per the PRISMA guidelines and 6285 articles screened. 60 randomized trials in LACC published during 1974-2018 without surgical interventions were selected. These used 13 different interventions-RT alone or/with neoadjuvant CT (NACT), adjuvant CT (ACT), concurrent CTRT (weekly CDDP/3-weekly CDDP/combination CT with CDDP/non-CDDP based CT), HypCS, Imm and HT. Random effects NMA were performed and odds ratios (ORs) estimated. Interventions for each endpoint were ranked as per their corresponding cumulative ranking curve (SUCRA) values. Quality of the evidence was evaluated according to the GRADE Working Group recommendations.

Results: Of the 9895 patients evaluated, $97.4 \%$ were LACC. Total events reported for LRC, OS, AM and LM were 5431/8197, $4482 / 7958,1710 / 7183$ and $441 / 6333$ respectively. The strategies with best ORs and 95\% credit intervals for LRC, OS, AM and LM were HT+RT (1.23, 0.49-3.19), concurrent CTRT (3-weekly CDDP) (1.14, $0.35-3.65), \mathrm{RT}+\mathrm{ACT}(0.01,0.00-1.04)$ and NACT+RT+ACT $(0.42$, $0.02-7.39)$ respectively. The three highest ranked comprehensive SUCRA values for all four endpoints taken together were HT+RT, HT+CTRT and CTRT (3-weekly CDDP). Further, two-step cluster analysis grouped these three interventions into a single distinctive cluster.

Conclusion: Using NMA, the greatest panoptic impact on key clinical endpoints in LACC was evident for HT+RT, HT+CTRT and CTRT (3-weekly CDDP). A phase III randomized trial between these shortlisted therapeutic strategies is thus warranted for a direct head-to-head comparison. Posterwalk

\section{The improvement of multidisciplinary discussion for the endometrial cancer treatment}

Simona Cima, C. Azinwi, D. Bosetti, I. Donegani, I. G. Maddalena, F. Martucci, G. Pesce, MC. Valli, A. Richetti

\section{Radiation Oncology Clinic, IOSI, Bellinzona, Switzerland}

Aims: To evaluate the alignment with the international guidelines in the adjuvant treatment of endometrial cancer patients after Multidisciplinary Discussion Meeting (MDM).

Methods: From 2015 to 2017133 new cases of endometrial cancer were recorded in Ticino and 117 cases $(88 \%)$ were discussed at weekly MDM of the Ente Ospedaliero Cantonale (EOC). During MDM gynecologists, medical oncologists, radiation oncologists, radiologists and pathologists discussed together for a shared therapeutic proposal. Ninety patients with endometrioid carcinoma were selected and divided into risk groups according to ESMO-ESGO-ESTRO recommendations, considered as reference.

Results: The $84 \%$ of pts, presented at MDM by reference gynecologist, underwent surgery in a single center. Risk group was: low or intermediate, 45 pts (50\%); high intermediate N0, 16 pts (18\%); high intermediate $N$ x, 2 pts (2\%); high stage I-II, 7 pts (8\%); high stage III, 17 pts (19\%); high stage IV, 3 pts (3\%). The RT and CT were performed in two different hospitals by the same dedicated staff. For all the cases, the relevance of adjuvant treatment to the guidelines was $91 \%$ : $100 \%$ for low and intermediate risk pts; $94 \%$ for high intermediate risk N0 pts; $50 \%$ for high intermediate risk $N$ x pts; $86 \%$ for high risk stage I-II pts; $71 \%$ for high risk stage III pts and $100 \%$ for high risk stage IV pts. The reasons of treatment omission were: old age in 2 cases, refusal in 2 cases, disease progression and development of a second tumor in 2 and 1 case, respectively. In 1 pts with high intermediate risk (N0) the RT on pelvis was prescribed due to isolated tumor cells in one of the sentinel lymph nodes analyzed and lymph-vascular space invasion. Two pts with stage IV disease were treated with chemotherapy and one with hormonal therapy.

Conclusion: ESMO-ESGO-ESTRO recommendations were applied in $91 \%$ of cases. The MDM allowed a better centralization of the oncologic gynecologic cases, especially for complex surgical procedures and following adjuvant treatments. Poster
Hypo- vs. normofractionated irradiation in early-stage breast cancer-A patterns of care analysis in German speaking countries

Mayinger Michael

Radiation Oncology, Universitätsspital Zürich, Zurich, Switzerland

Objective: Adjuvant radiotherapy (RT) plays an important role in early breast cancer management but the dose and fractionation schedules used are variable. A whole breast irradiation of $50 \mathrm{~Gy}$ in 25 daily fractions delivered over 5 weeks, usually followed by a boost is often considered the "standard" adjuvant RT prescription. Studies indicate that hypofractionated regimes such as 40.05 Gy in 15 daily fractions WBI are equally effective and achieve similar or better cosmetic and normal tissue outcomes. Thus, the 2017 German guidelines recommend hypofractionated RT (HF-RT) as a new standard of treatment for early breast cancer. However, there are limitations to the HF-RT studies such as the length of follow up. To understand the patterns of care in German-speaking countries a survey was conducted regarding the use of normo- and hypofractionated radiotherapy techniques.

Methods: Between July 2017 and August 2017, an email based survey was sent to all 1408 members of the German society of radiation oncology (DEGRO). The survey was completed by 180 physicians including 52 head of departments and 10 private practice owners.

Results: The majority of the 180 physicians who completed the survey use the normofractionated regimen of RT as standard treatment for early breast cancer $(76.6 \%)$. Several physicians are sceptical about HFRT and 7 physicians completely refused to use HF-RT. Many professionals do not agree (25.6\%) on the new German guidelines suggesting HF-RT as standard treatment for all patients, or adopted a neutral position $(32.8 \%)$ towards them. The main reasons for not aligning with the new guidelines are concerns about increased side effects and poorer treatment related outcome. Most physicians who perform HF-RT do so in an individual based manner (77.2\%).

Conclusion: HF-RT remains controversial in German speaking countries. Our data shows that NF-RT remains the predominant method of treatment. HF-RT is only used in a defined group of patients as most German physicians agree that particular patients, especially those at higher risk of RT late effects, may benefit from a less intense, extended fractionation schedule. Poster

\section{First case report of concurrent proton therapy with hyperthermia in a large extra-abdominal desmoid tumor}

E. Stutz, F. Murray ${ }^{2}$, E. Puric ${ }^{1}$, R. Schneider ${ }^{2}$, M. Walser ${ }^{2}$, A. Meister ${ }^{1}$, D. Marder ${ }^{1}$, O. Timm 1 , S. Rogers ${ }^{1}$, C. Britschgi ${ }^{3}$, DC. Weber ${ }^{2}$, S. Bodis ${ }^{1}$, NR. Datta ${ }^{1}$

${ }^{1}$ Center for Radiation-Oncology KSA-KSB, Aarau, Switzerland ${ }^{2}$ Center for Proton Therapy, PSI, Villigen, Switzerland

${ }^{3}$ Department of Hematology and Oncology, Universitätsspital Zürich, Zurich, Switzerland

Aims: We report the preliminary outcome of a large, extra-abdominal desmoid tumor (DT) of the right upper leg, 16 months after definitive treatment with concurrent proton therapy (PT) and hyperthermia (HT). Background: DTs are rare, semi-malignant tumors. Possible treatment options include systemic treatment (sTx), surgery and radiotherapy (RT). HT is a potent radiosensitizer in solid malignancies, but little is known about its effect in DTs. Isolated published data suggest that thermo-radiotherapy could be superior to RT alone (Tkachev et al., 2004, Vopr Oncol).

Methods: A 33-year old female patient presented with a large $(15.5 \times 12 \times 10 \mathrm{~cm})$ DT. Despite previous sTx with sulindac and tamoxifen, the tumor volume tripled to $806 \mathrm{cc}$ within 1.5 years. Surgery was not feasible. She was therefore treated with a total dose of 64.8 Gy $($ RBE) $(27 \times 2$ Gy + SIB at 2.4 Gy) with $7 \times$ weekly deep HT treat- 
ments prior to PT (max. simulated temperature: $44^{\circ} \mathrm{C}$ ). The tumor was contoured on 9 consecutive MRIs (T2+T1 contrast) and volumes were plotted in relation to time. The response was evaluated based on volume reduction and signal alteration.

Results: 16 months after completion of PTHT, a tumor volume regression of $44 \%$ ( 806 to $455 \mathrm{cc}$ ) with a mean decrease velocity of $22 \mathrm{cc} /$ month was noted. In parallel, a decrease in MRI signal intensity (T2) and contrast enhancement (T1) was observed. Subjectively, the feeling of tension and pain disappeared, and knee flection was less restricted. Except for a transient local erythema (CTCAE Grade 1), no acute or late toxicity occurred.

Conclusion: The decrease in volume, signal intensity and contrast enhancement can be interpreted as a tumor regression with associated intra-tumoral necrosis. As DTs are known to have slow regression, this and the observed necrosis suggest, that further tumor regression could be expected. PTHT may be a treatment option in large inoperable DTs after failure of sTx. Poster

\section{Retrospective evaluation of patients with early glottic cancer treated with radiotherapy only}

Thierry Spielmann, Jens Jakscha, Frank Zimmermann

Clinic of Radiotherapy and Radiation Oncology; Clinic of ORL, University Hospital Basel (USB), Basel, Switzerland

Aims: Definitive radiation therapy (RT) is one standard treatment for early stage squamous cell carcinoma of the larynx (SCCL). We evaluated retrospectively overall survival (OS), disease-free survival (DFS), location of tumor recurrence, and larynx-preservation-rate (LPR) of RT in patients with stages cT1-2 cN0 cM0 SCCL at the USB being treated between 1999 and 2017.

Methods: We analysed the electronic health records including treatment plans using a biological equivalent dose (BED; for tumor control: a/b-value of 10 ; for late effects: $a / b$-value of 3 ). We correlated the rate and location of tumor recurrence, OS, DFS and LPR with tumor stage, BED, dose distribution, treatment technique, planning efforts to protect the contralateral vocal cord. The survival rates were calculated according to Kaplan-Meier.

Results: Of in total 42 patients (mean age: 76 ), 17 had cT1 and 25 cT2 glottic/supraglottic cancer with $97.5 \%$ smokers, $83 \%$ men. 7 patients have been lost to follow-up. RT has been conducted with hyperfractionated schedules in $71 \%$. With a mean follow-up of 5.25 years, we observed 8 recurrences ( 7 local, 1 regional, 0 distant) and 10 secondary carcinomas. The $2-y-O S$ is $77 \%$, the 5 -y-OS is $65 \%$. The overall LPR is $75 \%$. Deaths due to secondary cancer occurred in $16 \%$. Techniques (3-D-conformal, IMRT, VMAT) and selective contralateral cord protection seem to have no impact on oncological outcome. BED of more than $87 \mathrm{~Gy}(\mathrm{a} / \mathrm{b}=3)$ seem to improve local control rate $(11 \%$ local recurrence vs. $21 \%$ local recurrence).

Conclusion: The local control rate was lower than in recently published trials. It might be explained by lower BED (mean: 85.4), which has been increased stepwise in the last years. The effects of dose escalation on oncological outcome and larynx-preservation will be observed in future within a prospective collection of data in an electronic database. Poster

\section{Impact of regular MRI follow-up after radiotherapy to the surgical cavity in patients with 1 to 3 brain metastases}

Bachmann N. ${ }^{1}$, Leiser D. ${ }^{1}$, Ermis E. ${ }^{1}$, Vulcu S. ${ }^{2}$, Schucht P. ${ }^{2}$, Raabe A. ${ }^{2}$, Aebersold DM. ${ }^{1}$, Herrmann E. ${ }^{1}$

${ }^{1}$ Department of Radiation Oncology, Inselspital, Bern University Hospital and University of Bern, Bern, Switzerland

${ }^{2}$ Department of Neurosurgery, Inselspital, Bern University Hospital and University of Bern, Bern, Switzerland
Aims: To report the outcome of patients with 1 to 3 brain metastases (BMs) treated with SRS or hypo-fractionated stereotactic radiotherapy (HFSRT) to the surgical cavity, with a specific aim to examine the impact of regular MRI follow-up (FU).

Methods: We retrospectively analyzed patients who received SRS or HFSRT to the tumor bed after resection of 1 to 2 BMs. Additional, non-resected BMs were managed with SRS or HFSRT only. Patients with any prior irradiation of the head were excluded. Survival was estimated by the Kaplan-Meier method. Prognostic factors were examined with log-rank test, Cox proportional hazards model and the Mann-Whitney U test. Primary endpoint was LC. Secondary endpoints were DBC, OS and the correlation between regular MRI FU and OS, symptom-free survival (SFS), deferment of WBRT and WBRT-free survival (WFS).

Results: Overall 75 patients were enrolled. One, 2 and 3 BMs were seen in $84 \%(n=63), 15 \%(n=11)$ and $1 \%(n=1)$ of the patients, respectively. Regular MRI FU was performed in 53\% $(n=40)$ of the cases. Median OS was 19.4 months (95\% CI: 13.2-25.6 months). Actuarial LC, DBC and OS at 1 year were 72\% (95\% CI: 60-83\%), 60\% (95\% CI: $48-72 \%)$ and $66 \%$ (95\% CI: 53-76\%), respectively. A PTV $>15 \mathrm{c}^{\mathrm{m} 3}$ $(p=0.005)$, biologically effective dose (BED) $<40$ Gy $(p=0.018)$, tumor rest after surgery $(p=0.001)$, progressive neurological symptoms $(p=0.012)$ and progressive extracranial tumor status $(p=0.001)$ revealed significant decreased OS. No significant correlation between regular MRI FU and OS ( $p=0.462)$, SFS $(p=0.536)$, WFS $(p=0.407)$ and deferment of WBRT ( $p=0.955)$ could be shown.

Conclusion: Our results regarding oncological outcomes consist with the current literature data. Surprisingly, regular MRI FU has not resulted in increased OS, SFS, WFS or deferment of WBRT in our cohort consisting mainly of patients with a single BM. Therefore, the impact of regular MRI FU needs prospective evaluation. Oral presentation

\section{CT radiomics to predict outcome in patients with malignant pleural mesothelioma}

M. Pavic ${ }^{1 *}$, J. Kraft ${ }^{1 *}$, S. Tanadini-Lang ${ }^{1}$, M. Bogowicz ${ }^{1}$, D. Vuong ${ }^{1}$, S. Kroeze ${ }^{1}$, M. Friess ${ }^{2}$, T. Frauenfelder ${ }^{3}$, N. Andratschke ${ }^{1}$, M. Guckenberger ${ }^{1}$, W. Weder ${ }^{2}$, I. Opitz ${ }^{2}$

*both authors contributed equally

${ }^{1}$ Department of Radiation Oncology, University Hospital Zurich, University of Zurich, Zurich, Switzerland

${ }^{2}$ Department of Thoracic Surgery, University Hospital Zurich, University of Zurich, Zurich, Switzerland

${ }^{3}$ Department of Radiology, University Hospital Zurich, University of Zurich, Zurich, Switzerland

Aims: Tumor volumetry is identified as an important prognostic factor (PF) for OS of mesothelioma patients. Radiomics is a mathematical method to quantify shape, intensity and texture of a tumor on medical images in a more comprehensive way as compared to volumetry. We aimed to evaluate radiomic features (RF) in CT images as a PF for OS in patients with malignant pleural mesothelioma (MPM).

Methods: Primary tumor was delineated in 30 patients with proven MPM in CT images performed at the time of primary diagnosis. 1404 RF of shape $(n=18)$, histogram $(n=17)$, texture $(n=137)$ and wavelet $(n=1232)$ were calculated with an in-house developed software (Z-Rad). RF were pre-selected based on an inter-observer delineation robustness study and stable RF were grouped based on average hierarchical clustering. Per group of correlated features, the most prognostic one was selected and included in multivariable Cox regression for prediction of OS and progression free survival (PFS) calculated from diagnosis.

Results: Median follow up was 14.1 months and median OS 14.2 months. 505 of the RF were stable and hierarchical clustering revealed 6 groups of correlated and stable RF. In total, $18 \mathrm{RF}$ distributed over 5 groups of correlated features were prognostic in univariable Cox re- 
gression and two wavelet features were prognostic for OS in multivariable Cox regression (concordance index 0.74, $p=0.002$ ). Both features separated the patients into two groups with a significant different OS and were also significant for PFS. For comparison, tumor volume was prognostic in univariable Cox regression $(p=0.01)$ but had a significantly lower concordance index (0.62).

Conclusion: Radiomic biomarkers had a stronger prognostic value in terms of OS compared to a traditional model based on tumor volume. Oral presentation

\section{Synchronous bilateral breast cancer radiotherapy using VMAT and deep inspiration breath hold (DIBH)}

S. Cima ${ }^{1}$; D. Gaudino ${ }^{2}$; C. Azinwi ${ }^{1}$, D. Bosetti ${ }^{1}$, I. Donegani ${ }^{1}$, I. G. Maddalena ${ }^{1}$, F. Martucci ${ }^{1}$, G. Pesce ${ }^{1}$; S. Presilla ${ }^{2}$, MC. Valli ${ }^{1}$, A. Richetti ${ }^{1}$

${ }^{1}$ Radiation Oncology, Oncology Institute of Southern Switzerland IOSI, Bellinzona, Switzerland

${ }^{2}$ Medical Physics Unit, EOC, Bellinzona, Switzerland

Purpose: The aim of this study was to investigate the feasibility and skin toxicity in Synchronous Bilateral Breast Cancer (SBBC) patients treated with adjuvant radiotherapy (RT) using Volumetric Modulated Arc Therapy (VMAT) and Deep Inspiration Breath Hold (DIBH).

Material and methods: 15 patients were treated from January 2013 to July 2017 to a dose of 50.4 Gy prescribed to the whole bilateral breasts + - - regional lymph nodes $\left(\mathrm{PTV}_{\mathrm{II}}\right)$, according to the stage and $64.4 \mathrm{~Gy}$ to the surgical beds $\left(\mathrm{PTV}_{\mathrm{I}}\right)$ via a simultaneous integrated boost (SIB). The daily doses were 1.8 and 2.3 Gy for each PTV, respectively. The dosimetric parameters analysed were $\mathrm{D}_{95 \%} ; \mathrm{D}_{100 \%} ; \mathrm{V}_{105 \%}$ for the PT$\mathrm{Vs} ; \mathrm{V}_{30}$ and mean dose for both lungs; $\mathrm{D}_{\max }$ for the left anterior descending coronary artery (LAD); $\mathrm{D}_{\max }$ and mean dose for the heart; $\mathrm{D}_{\max }$ for the spinal cord. Skin toxicity was recorded during and after treatment, based on the RTOG acute radiation morbidity scoring criteria.

Results: The median age of the population was 65 years (range 31-78). All patients were alive without any sign of disease, at a median follow-up of 22 months. The maximum acute skin toxicity registered at the end of

Table 1

\begin{tabular}{ll}
\hline Parameter & Median \pm DS \\
\hline PTVII & $1681.4 \pm 441.2$ \\
Volume & $96.2 \pm 1.4$ \\
D95 \% & $37.9 \pm 7.6$ \\
D100\% & $0.02 \pm 0.08$ \\
V105\% & \\
PTVI & $150.9 \pm 72.7$ \\
Volume & $98.7 \pm 0.7$ \\
D95 \% & $57.7 \pm 1.0$ \\
D100 \% & $0.2 \pm 0.5$ \\
V105 \% & \\
Lungs & $8.3 \pm 1.8$ \\
V30 \% & $13.3 \pm 1.4$ \\
D mean & \\
LAD & $14.3 \pm 3.3$ \\
Dmax & \\
Heart & $6.5 \pm 1.6$ \\
D mean & $19.2 \pm 5.1$ \\
Dmax & \\
Spinal cord & $9.8 \pm 2.3$ \\
Dmax &
\end{tabular}

treatment was Grade 1 in 12 patients and Grade 2 in 3 patients. No Grade 3 or 4 toxicity was observed. No late skin toxicity was registered except Grade 1 in 3 patients. Dosimetric results are summarized in Table 1.

Conclusion: The present retrospective analysis shows the feasibility of VMAT in the treatment of SBBC patients. The data, with regards to acute and late toxicity, confirm the safety of the advanced technique used. Furthermore, a long term follow up is needed in order to assess clinical outcomes. Poster

\section{Thermo-radiotherapy in bladder cancer is a promising option in patients unfit for cystectomy or chemo-radiotherapy}

E. Stutz ${ }^{1}$, B. Eberle ${ }^{1}$, E. Puric ${ }^{1}$, A. Meister ${ }^{1}$, O. Timm ${ }^{1}$, D. Marder ${ }^{1}$, S. Rogers ${ }^{1}$, S. Wyler ${ }^{2}$, NR. Datta ${ }^{1}$, S. Bodis ${ }^{1}$

${ }^{1}$ Center for Radiation-Oncology KSA-KSB, Aarau, Switzerland ${ }^{2}$ Department of Urology, KSA, Aarau, Switzerland

Aim: To evaluate the outcome of radiotherapy with concomitant deep hyperthermia (HTRT) in muscle-invasive bladder cancer (MIBC) patients, who are not fit for or decline radical cystectomy (RC) or chemo-radiotherapy (CTRT).

Methods: 17 patients with unifocal or multifocal MIBC (T1-4, cN0, cM0, G3) treated from $12 / 2012$ to $03 / 2018$ with a trimodal treatment (TMT) of initial TUR-B and HTRT, were evaluated. Multifocal MIBCs received $50 \mathrm{~Gy} / 20 \mathrm{fx}(5 \mathrm{x} /$ week $)$ to the whole bladder and unifocal MIBCs $48 \mathrm{~Gy} / 16 \mathrm{fx}$ (4x/week) in total, $36 \mathrm{~Gy} / 12 \mathrm{fx}$ to the full bladder and $12 \mathrm{~Gy} / 4 \mathrm{fx}$ boost to the former tumor region. HT was administrated weekly 4-5 times over $60 \mathrm{~min}$ with a mean temperature of $41.3{ }^{\circ} \mathrm{C}$. Results: One patient did not tolerate HTRT and was excluded. $94.1 \%$ completed TMT as per protocol. These 16 patients ( 6 unifocal, 10 multifocal) had a median age of 81 years and a median age-adjusted Charlson comorbidity index of 5. All patients had local control (LC) at 6 weeks cystoscopic follow-up (c-f/u). 2 patients have not yet reached the 6-months $\mathrm{c}-\mathrm{f} / \mathrm{u}$ and 5 patients died without or prior to 6-months c-f/u (at 4, 6, 6, 7, 23 months, not MIBC-related), but with clinical LC. Thus, $9 / 9$ patients $(100 \%)$ showed LC at 6-months c-f/u. Life-time LC was achieved in 14/16 patients $(87.5 \%)$ with a median c-f/u of 7.5 months and a median clinical f/u of 11.4 months (both range 2-59). 6 patients died (none MIBC-related). One local relapse was detected at 9.5 months (rpTa) and was successfully salvaged with TUR-B. The other was discovered at 17 months with lymph node and bone metastases. Acute toxicity grade 3 (CTCAE 4.03) was observed in 2/16 patients $(12.5 \%)$. One had constipation with no clear relationship to HTRT. Only two late toxicities grade $\geq 2$ occurred $(1 \times$ GI grade $2,1 \times$ transient GU grade 3). Bladder function was well preserved in all patients.

Conclusion: In elderly and polymorbid patients not fit for curative RC or CTRT, HTRT appears to be an effective, well tolerated treatment providing good LC without relevant toxicities. Oral presentation

\section{Definitive radiotherapy of PSMA-PET-positive oligo-metastatic} recurrent prostate cancer: A single-center experience

S. Kroeze ${ }^{1}$, I. A. Burger ${ }^{2}$, H. Garcia ${ }^{1}$, T. Hermanns ${ }^{3}$, M. Guckenberger

${ }^{1}$ Department of Radiation Oncology, University Hospital Zürich, Zurich, Switzerland

${ }^{2}$ Department of Nuclear Medicine, University Hospital Zürich, Zurich, Switzerland

${ }^{3}$ Department of Urology, University Hospital Zürich, Zurich, Switzerland

Introduction: Recurrence rates after PCa treatment are $\sim 40-80 \%$. Although salvage RT of the prostate bed is the standard of care, $20-40 \%$ of recurrences are located outside this area. PSMA-PET has become available for identification of recurrences at low PSA-levels, and RT of PSMA-positive oligometastatic recurrences (ORT) has become a frequently practiced option to improve prognosis or delay a start of ADT. 
Materials and Methods: Data of 51 patients with oligometastatic PSMA-positive PCa recurrences who underwent ORT of all metastatic sites between 06/2016-01/2018 in one academic center were retrospectively evaluated. Most (86\%) did not receive ADT. RT planning, PSA-progression free survival (PSA-PFS) and toxicity were analyzed. Results: Median age was 64y (49-85), 63\% were high-risk, 67\% were R1. Median PSA at time of PET was $0.82(0.04-22.1) \mathrm{ng} / \mathrm{ml}$. PSA-DT was median $6(0-48)$ mo. Median nr of irradiated metastases was 2 (111): 30 lymph node (LN), 10 bone (BM) and 1 lung metastases. 13 had PSMA+ prostate bed recurrences. Median dose (EQD2/1.5) to prostate bed recurrences was $72(63.4-83) \mathrm{Gy}$ and to $\mathrm{LN}+65.8$ (46.4-98.6)Gy. Elective pelvic lymphatic irradiation was performed in all patients with $\mathrm{LN}+$ and combined with a SIB. BM and the lung metastasis were stereotactically irradiated with median 85 (62.8-108.6)Gy. Median FU was 7 (0-15) mo. 89\% showed a PSA response. PSA-recurrences (5/44) occurred after median $9 \mathrm{mo}(3-15)$. In the cohort w/o ADT, average PSA dropped significantly from $4.55(0.14-27.3)$ to $0.45(0-8.06) \mathrm{ng} / \mathrm{ml}$ $(p=0.001) 1-11 \mathrm{mo}$ after ORT. With ADT, mean PSA dropped from $0.96(0.12-2.49)$ to $0.05(0-0.13) \mathrm{ng} / \mathrm{ml}(p=0.06) 1-12 \mathrm{mo}$ thereafter. PSA-PFS at $6 \mathrm{mo}$ was $44 \%$. Acute toxicity consisted of $1 \mathrm{Gr} 2$ urinary frequency and $1 \mathrm{Gr} 2$ diarrhea.

Conclusions: Our preliminary experience in this cohort of patients, is that PSMA-targeted RT is feasible and safe. Although the follow-up was short, at 6 months approximately $50 \%$ presented with regredient PSA-levels. Irradiating PSMA-positive oligometastatic PCa recurrences might play a role in prolonging the time to start of ADT. Prospective trials are needed to select patient subgroups that would benefit most from this treatment. Oral presentation

\section{Clinical outcome of arteriovenous malformations treated with radiosurgery-A single center institutional analysis}

Blatti-Moreno $\mathrm{M}^{1}$, Stieb S ${ }^{1}$, Vlaskou-Badra $\mathrm{E}^{1}$, Ermis $\mathrm{E}^{1}$, Aebersold $\mathrm{DM}^{1}$, Gralla $\mathrm{J}^{2}$, Mordasisni $\mathrm{P}^{2}$, Raabe $\mathrm{A}^{3}$, Bervini $\mathrm{D}^{3}$, Herrmann $\mathrm{E}^{1}$

${ }^{1}$ Department of Radiation Oncology, Inselspital, Bern University Hospital and University of Bern, Bern, Switzerland

${ }^{2}$ Department of Neuroradiology, Inselspital, Bern University Hospital and University of Bern, Bern, Switzerland

${ }^{3}$ Department of Neurosurgery, Inselspital, Bern University Hospital and University of Bern, Bern, Switzerland

Aims: To report the outcome of patients with arteriovenous malformations (AVM) treated with stereotactic radiosurgery (SRS) at our center. Methods: We retrospectively analyzed adult and pediatric patients with an AVM that were treated with LINAC and Cyberknife SRS. Patients with $<3$ months follow-up (FU) were excluded. Time to event for obliteration rate (OR) was estimated using the Kaplan-Meier method. Fisher's exact test was used to test for significant differences in OR regarding patient and treatment characteristics. Significance level was set as $\leq 0.05$. Primary endpoint was OR after SRS and secondary endpoints were toxicity and symptom worsening.

Results: Overall 27 patients were included. Mean age was 43.6 years (SD 17.8). Initial clinical presentation at diagnosis was hemorrhage in $n=21(77.8 \%)$ and seizures in $n=6$ patients $(22.2 \%)$. Most common Spetzler-Martin grade was III $(n=15,55.6 \%)$. Prior embolization and surgery was performed in $n=17(63.0 \%)$ and $n=5$ patients $(18.5 \%)$. Median radiological FU was 36 months (range 4-167). Mean target volume was 3.3cc (SD 3.1) and mean dose was 18.5 Gy (12-20, SD 2.1 ), prescribed to a mean $85 \%$ isodoseline (range $57-98 \%$ ). Five patients $(18.5 \%)$ were re-treated by SRS. OR at 1, 2, 3, 4 and 5 years was $4,18,33,43$ and $49 \%$. Clinical complications occurred in $n=5$ patients (18.5\%), including $n=1$ post-treatment hemorrhage (3.7\%). Additional data will be presented at the meeting.

Conclusion: Our preliminary results show an obliteration rate of AVMs after SRS in the range of reported outcomes in the literature. Poster
Isotoxic and isoeffective stereotactic re-irradiation for high grade gliomas

S. Rogers, N. Lomax, S. Alonso, S. Khan, E. Rabe, A. Meister, S. Bodis

Radio-Onkologie-Zentrum KSA-KSB, Aarau, Switzerland

Aims: There is no standard dose fractionation for the re-irradiation of HGG beyond $\mathrm{NTD}_{\text {cumulative }}<100$ Gy to minimise brain radionecrosis (RN). Recent patients had small volume recurrences and we increased fraction size and reduced fraction number, based on OAR V(brainGTV), to maintain a low risk of RN.

Methods: Our standard is (1) $10 \times 3.5 \mathrm{~Gy}$, PTV $=$ GTV up to $100 \mathrm{~cm}^{3}$. For volumes $0.3-4.3 \mathrm{~cm}^{3}$, we prescribed (2) $5 \times 6$ Gy or (3) $1 \times 18$ Gy according to volume and location. The normal brain-GTV constraints were (2) $\mathrm{V}_{20 \mathrm{~Gy}}=20 \mathrm{~cm}^{3}$ and $(3) \mathrm{V}_{10 \mathrm{~Gy}}=10 \mathrm{~cm}^{3}$. SRT/RS was planned with VMAT (1) and DCAs (2 and 3) and was delivered with a Novalis STx linac (Brainlab/Varian).

Results: The $\mathrm{BED}_{10}$ for schedules (1), (2) and (3) are $47.8 \mathrm{~Gy}, 48$ Gy and 50 Gyand thus biologically isoeffective. The $\mathrm{BED}_{2}$ are $96 \mathrm{~Gy}, 120$ Gy and 180 Gyrespectively. 11 consecutive patients were analysed: Combs prognostic score $2-3$, age $42-72$ yrs, KPS $>90,8$ primary and 2 secondary GBM, 1 Grade III astrocytoma. The median time to re-RT after 60 Gy was 5 yrs (range $0.5-13$ yrs), 2 had salvage surgery and 1 GTV $>47 \mathrm{c}^{\mathrm{m} 3}$ (mean 17.1, range $0.3-100.9 \mathrm{~cm}^{3}$ ). 7 were treated with schedule (1) and 4 with schedules (2) and (3). Mean $V_{20 G y}$ was $4.9 \mathrm{~cm}^{3}$ and $\mathrm{V}_{10 \mathrm{~Gy}}$ was $1.7 \mathrm{~cm}^{3}$. Follow-up MRIs are available for 10 patients with med. follow-up $5.5 \mathrm{mths}$ ( $2-58 \mathrm{mths}) .2$ patients have died, med. OS since diagnosis was 6 yrs (1.5-30 yrs). Median time to progression and next line of therapy was $3.5 \mathrm{mths}(2-41 \mathrm{mths})$. No patients had symptomatic necrosis and steroids were successfully discontinued. Conclusion: Not only patient selection but also comparable BEDs may account for the similar control rates between published schedules. We have adopted a risk-adapted approach to maintain efficacy and minimise RN. Our initial approach to isotoxic re-irradiation of HGG based on V(brain-GTV) is GTV $\leq 1 \mathrm{c}^{\mathrm{m} 3}: 1 \times 18-20 \mathrm{~Gy}, 1-5 \mathrm{~cm}^{3}: 5 \times 6$ Gy and $>5 \mathrm{c}^{\mathrm{m} 3}: 10 \times 3.5 \mathrm{~Gy}$. The first patients treated without radiation-induced toxicity suggest the feasibility of this approach. Poster

\section{Concurrent chemoradiation with Volumetric Modulated Arc Therapy in anal cancer patients: Acute and late toxicity}

I. G. Maddalena, S. Cima, F. Martucci, D. Bosetti, C. Azinwi, GA Pesce, M. I. Donegani, A. Richetti, MC. Valli

Radiation Oncology, Oncology Institute of Southern Switzerland IOSI, Bellinzona, Switzerland

Aim: To report the acute and late toxicities and the clinical outcome of patients treated for anal cancer with Volumetric Modulated Arc Therapy (VMAT)/RapidArc and concomitant chemotherapy.

Patients and methods: A cohort of 30 patients with histologically confirmed squamous cell carcinoma of the anal canal were treated in our clinic from 2010 to 2017 . We treated all the patients in supine position. The dose prescribed to elective target volume (PTVII) was 39.6 Gy while 54-59.4 Gy were prescribed to primary tumor and metastatic lymph nodes (PTVI). In case of Simultaneous Integrated Boost (SIB) the dose prescribed to PTVII and PTVI varied from 42-45 Gy and 50.4-54 Gy respectively, according to stage. Concomitant chemotherapy consisted of Mitomycin C and 5-Fluorouracil or Capecitabine.

Patients were checked clinically and radiologically every 4 months for the first 2 years and then biannually. The acute and late toxicities were registered according to EORTC/RTOG scales.

Results: Median follow-up was 44.9 months. The 3 year OS was $88.4 \%$ and LRC was $88.6 \%$.

Grade 3 acute dermatological toxicity was registered in 1 patient (3.3\%), Grade 2 in 17 patients $(56.6 \%)$ and Grade 1 in 10 patients 
$(33.3 \%)$. Grade 2 gastrointestinal (GI) toxicity was registered in 4 patients $(13.3 \%)$ while Grade 1 in 16 patients $(53.3 \%)$. Grade 1 acute genitourinary (GU) toxicity was recorded in 15 patients $(50 \%)$.

Grade 2 late dermatological toxicity was detected in 2 patients $(6.6 \%)$ while Grade 1 in 2 patients (3.3\%). No patient experienced late GU toxicity. Three patients (10\%) experienced Grade 1 late GI toxicity. One patient experienced grade 3 incontinence of the anal sphincter while 2 experienced grade 1 incontinence (3.3\%). No patient experienced anal sphincter stenosis.

Conclusion: Our results support VMAT as safe and effective radiotherapy technique in the treatment of anal canal cancer. Poster

\section{Stereotactic body radiotherapy for refractory ventricular tachycardia: The Swiss experience}

R. Jumeau ${ }^{1}$, M. Ozsahin ${ }^{1}$, J. Schwitter ${ }^{2}$, F. Duclos ${ }^{1}$, V. Vallet ${ }^{3}$, M. Zeverino ${ }^{3}$, R. Moeckli ${ }^{3}$, E. Pruvot ${ }^{2}$, J. Bourhis $^{1}$

${ }^{1}$ Departement of Radiation Oncology, Centre Hospitalier Universitaire Vaudois (CHUV), Lausanne, Switzerland ${ }^{2}$ Department of Cardiology, Centre Hospitalier Universitaire Vaudois (CHUV), Lausanne, Switzerland

${ }^{3}$ Institute of Radiation physics, Centre Hospitalier Universitaire Vaudois (CHUV), Lausanne, Switzerland

Introduction: Stereotactic body radiotherapy (SBRT) is an emerging treatment for ventricular tachycardia (VT) refractory to antiarrhythmic drugs (AADs) and catheter ablation (CA). We present here the outcomes of our first patients treated under an institutional SBRT program for refractory VT.

Material and methods: Enrolled patients suffered from recurrent VT or electrical storms (ES) refractory to CA and AADs. Before the procedure, an electroanatomic mapping (EAM) was performed to localize the VT substrate (VT-sub). All patients underwent a planning CT co-registered with a cardiac MRI to help in volume definition. For each case, the cardiologist delineated the VT-sub according to the EAM data. The right ventricular defibrillation lead of the implantable cardioverter defibrillator (ICD) was used as a fiducial marker for tracking. A total dose of $25 \mathrm{~Gy}$ was delivered to the VT-sub using Cyberknife ${ }^{\circ}$. Results: Since September 2017, three patients with VT arising from the interventricular septum were treated. Two patients were elective, while another one, hospitalized in the intensive care unit (ICU), was intubated because of an ES with multiple ICD shocks. In all patients, SBRT was successfully delivered using near real-time ICD lead tracking. The VT-sub volume were $19 \mathrm{cc}, 21 \mathrm{cc}$ and $35 \mathrm{cc}$, respectively. After a median follow-up of 5 months, the elective patients did not experience any VT recurrence. The ICU patient was extubated 3 days after SBRT and remained free of ICD shocks during 4 months; he presented, however, a new ES 19 weeks after the procedure. This ES episode was related to a new VT-sub confirmed by EAM, and successfully treated by CA. Importantly, no SBRT-related toxicity occurred.

Conclusion: SBRT appears to be an efficient therapeutic tool for the treatment of refractory VT. SBRT can be delivered non-invasively with Cyberknife ${ }^{\circledR}$ using the tracking of ICD lead. Posterwalk

\section{Does a boost dose improve local control for certain subgroups of early breast cancer patients older than $\mathbf{5 0}$ years?}

C. Vrieling ${ }^{1}$, E. van Werkhoven ${ }^{2}$, H. Bartelink ${ }^{2}$

${ }^{1}$ Departement: Radiation Oncology, Clinique des Grangettes, Geneva, Switzerland

${ }^{2}$ Departement: Radiation Oncology, The NKI, Amsterdam, The Netherlands

Background: The EORTC "boost no-boost" trial showed that young age and grade 3 invasive carcinoma were important risk factors for ipsilateral breast tumor recurrence (IBTR). This study re-analyses prog- nostic factors related to IBTR with long-term follow-up, with a special focus on the population over 50 years of age.

Methods: Patients with microscopically complete excision for invasive disease followed by whole-breast irradiation of $50 \mathrm{~Gy}$ in 5 weeks were randomised to receive 16 Gy boost or no boost. A central pathology review of 1616 patients was used for the current analysis. Median follow-up was 18.2 years. Results with a $p$-value $<0.01$ were considered statistically significant.

Results: 160 events were observed. Young age and presence of DCIS were associated with an increased risk of IBTR in multivariable analysis. The cumulative incidence (CI) of IBTR at 20 years was $34 \%$ for patients 40 years or younger, $14 \%$ for those 41 to 50 and $11 \%$ for patients over 50 years of age $(p<0.001)$. The CI was $18 \%$ and $9 \%$ for tumors with and without DCIS $(p<0.001)$.

In the subgroup of patients older than 50 years of age, the boost did not change the 20 -year CI of IBTR ( $11 \%$ with boost, $12 \%$ without boost). The invasive tumor grade did not significantly change the 20year CI of IBTR ( $10 \%$ for grade $1 / 2$ and $14 \%$ for grade 3 ). For patients over 50 years with additional DCIS, the 20 -year CI of IBTR was $15 \%$ compared to $7 \%$ without DCIS ( $p=0.03$ ). However, for patients with DCIS, the boost did not change the 20 -year CI of IBRT (14\% with boost, $15 \%$ without boost). The final risk group analysed was the subgroup of patients over 50 years with hormone receptor negative, high grade tumors. The 15 -year CI of IBTR was only $6 \%$.

Conclusion: The boost to the tumor bed is an important part of radiotherapy treatment in patients younger than 50 years of age. However, for the patients over 50, a subgroup that benefitted from an additional 16-Gy boost could not be defined. Oral presentation

\section{Radiotherapy outcome in stage I-II glottic larynx cancer: A multicenter study}

O. Elicin ${ }^{1}$, E. Ermiş ${ }^{1,2}$, C. Oehler ${ }^{3}$, D. M. Aebersold ${ }^{1}$, F. Caparrotti ${ }^{4}$, F. Zimmermann ${ }^{5}$, G. Studer ${ }^{6,7}$, G. Henke ${ }^{8}$, L. Adam ${ }^{3}$, L. Anschuetz ${ }^{9}$, M. Guckenberger ${ }^{7}$, M. Shelan ${ }^{1}$, N. Kaydihan ${ }^{10}$, M. Ozsahin ${ }^{11}$, O. Riesterer ${ }^{7}$, T. Spielmann ${ }^{5}$, R. Giger ${ }^{9}$, M. Şen ${ }^{2}$

${ }^{1}$ Department of Radiation Oncology, Inselspital, University Hospital of Bern, Bern, Switzerland; ${ }^{2}$ Department of Clinical Oncology, St. James's Institute of Oncology, Leeds, Great Britain; ${ }^{3}$ Radiation Oncology Department, Cantonal Hospital of Graubunden, Chur, Switzerland; ${ }^{4}$ Radiation Oncology Department, University Hospital of Geneva, Geneva, Switzerland; ${ }^{5}$ University Hospital of Basel, Basel, Switzerland; ${ }^{6}$ Radiation Oncology Department, Cantonal Hospital of Lucerne, Lucerne, Switzerland; ${ }^{7}$ Radiation Oncology Department, University Hospital of Zurich, Zurich, Switzerland; ${ }^{8}$ Radiation Oncology Department, Cantonal Hospital of St. Gallen, St. Gallen, Switzerland; ' Department of Otorhinolaryngology, Head and Neck Surgery, University Hospital of Bern, Bern, Switzerland; ${ }^{10}$ Radiation Oncology Department, Istanbul University Cerrahpasa Faculty of Medicine, Fatih/İstanbul, Turkey; ${ }^{11}$ Radiation Oncology Department, University Hospital of Lausanne, Lausanne, Switzerland

Aims: The goal was to investigate the impact of radiotherapy (RT) dose and fractionation on patients' outcome in a cohort from 9 institutions.

Methods: Charts of 715 patients curatively treated with definitive RT between 1990 and 2015 were retrospectively reviewed. Day 0 was the date of diagnosis. The impact of potential variables on outcome was investigated by Cox proportional hazards models.

Results: Median follow-up was 63 months. Five-year loco-regional control (LRC) and overall survival (OS) were $83 \%$ and $84 \%$, respectively. Male/female* (HR: $4.38 ; 95 \%$ CI: $1.65-17.78 ; p=0.001$ ), stage II/I (HR: 1.62; 95\% CI: 1.10-2.36; $p=0.015)$ and anterior commissure involvement* (HR: 1.88 ; 95\% CI: $1.25-2.89 ; p=0.002$ ) were risk factors influencing LRC. Stage II/I* (HR: 1.68; 95\% CI: $1.21-$ $2.33 ; p=0.002$ ), fraction size $>2$ Gy (HR: $1.71 ; 95 \%$ CI: $1.96-2.41$; 
$p=0.004)$ and treatment time <45 days* (HR: 1.79 ; 95\% CI: 1.29 $2.49 ; p<0.001)$ were associated with lower OS $(*: p<0.05$ in multivariate models). Second cancer incidence was $10 \%$ and $22 \%$ in 5 and 10 years, respectively.

Conclusion: Contrary to the literature, our series did not show increased LRC with reduced treatment time. Hypofractionated and accelerated RT was associated with lower OS. These may be explained by possible differences in competing risk factors for death, staging procedures and selection bias for modified fractionation. Posterwalk

\section{Accelerated partial breast irradiation (APBI) with external beam radiotherapy-first experiences}

Stenger A., Unterkirhere O., Glanzmann C., Studer G.

Institute of Radiation Oncology, Cantonal Hospital Lucerne, Lucerne, Switzerland

Aims: To evaluate early outcome of APBI in early stage breast cancer patients.

Methods: APBI was implemented in our daily practice in February 2017. Inclusion criteria were defined according to the ASTRO CONSENSUS STATEMENT. Prescribed dose was 26 or 27 Gy, in 5 fractions on 5 consecutive days. A prospectively planned follow-up with objective and subjective assessment of treatment tolerance was performed after 0 and 2 weeks, 6 months and 1 year.

Results: Between 02/2017-04/2018, 67 patients met the inclusion criteria for APBI. $17 \%$ of patients were diagnosed with DCIS, 3/34/38\% with $\mathrm{T} 1 \mathrm{a} / \mathrm{b} / \mathrm{c}$ and $8 \%$ with $\mathrm{T} 2$ tumors. Mean PTV was $140 \mathrm{cc}$ (range 45-465), mean PTV to whole breast volume ratio 23\% (range 9-46). Mean age was 66 years (range 49-82). Mean follow-up was 152 days (range 10-393 days).

Acute skin toxicity after 2 weeks was low with $62 / 29 / 9 \%$ grade 0/1/2. 6-months follow-up data was available for 50 patients: $2 / 3$ and $1 / 3$ of patients reported no or minimal pain in the treated breast; $90 \%$ of patients had no change in skin sensitivity. Patient-reported cosmetic results after treatment were evaluated in $66 / 28 / 6 \%$ as excellent/good/ fair, respectively. 6-month treatment reactions were mild with fibrosis grade $0 / 1 / 2$ in $82 / 11 / 7 \%$. We observed grade 1 skin telangiectasia/ edema/atrophy in $4 / 11 / 7 \%$ of patients. Similarly, 18 patients who were examined after 1-year follow-up presented with no to minimal changes. There was no loco-regional recurrence diagnosed so far (04/2018). Conclusion: We herewith confirmed the high early and intermediate treatment tolerance of APBI in carefully selected early breast cancer patients. APBI is highly convenient for patients and economically efficient for busy centers. Based on data from other centers and the own tolerance results, the dose will be increased to $30 \mathrm{~Gy} / 5 \mathrm{fr} / 5 \mathrm{~d}$. Posteralk

\section{Fully automated segmentation of the brain resection cavity for radiation target volume definition in glioblastoma patients}

Herrmann $\mathrm{E}^{1}$, Ermis $\mathrm{E}^{1}$, Meier $\mathrm{R}^{3}$, Blatti-Moreno $\mathrm{M}^{1}$, Knecht $\mathrm{UP}^{2}$, Aebersold $\mathrm{DM}^{1}$, Manser $\mathrm{P}^{1}$, Reyes $\mathrm{M}^{3}$

${ }^{1}$ Department of Radiation Oncology and Medical Physics, University of Bern, Bern, Switzerland

${ }^{2}$ Department of Neuroradiology, Inselspital Bern University Hospital, University of Bern, Bern, Switzerland

${ }^{3}$ ISTB University of Bern, Bern, Switzerland

Objective: Our standalone software, using machine learning (ML) technology, performs fully automatic GBM segmentation in $5 \mathrm{~min}$. We evaluate if fully automated segmentation (FAS) of the resection cavity (RC) in GBM patients is feasible for RT target volume definition. Methods: Post-OP, pre- and post-contrast T1, T2 and FLAIR MRI datasets of 30 patients with surgically resected GBM were included. RCs were defined as surgical cavity plus blood products. Three radiation oncologists manually delineated the RC using all 4 MRI sets. For
FAS, we developed a cavity segmentation method. MRI data was skullstripped and co-registered for FAS. Manual delineations were utilized for training the ML system. A manual correction step was performed with naive brushing for the most obvious errors. We evaluated the segmentation method in terms of dice-coefficient (DC) and estimated volume measurements followed by a 3-fold cross-validation using the imaging data.

Results: Median DC and IQR of the different pairings of expert raters (reported as tuples) were $(0.85,0.08),(0.84,0.07)$, and $(0.86,0.07)$. The results of the FAS compared to the 3 raters were $(0.76,0.23)$, $(0.74,0.22)$, and $(0.73,0.19)$. After the manual correction step, the results improved to $(0.83,0.1),(0.8,0.1)$, and $(0.81,0.09)$. We did not detect a significant difference in distribution of the measured volumes for the different raters and methods (Kruskal-Wallis test: chi-square $=1.79$, $p=0.77$ ). Main sources of error was over- or under-segmentation of the RC due to signal inhomogeneity and over-segmentation due to similar intensity patterns.

Conclusions: The current prototype yields good results for the segmentation of the RC. Blood products and air can affect its performance. The computer-generated results are still subpar. Nonetheless, we generate first promising results. Oral presentation

\section{Functional outcome in laryngeal cancer: A single-center retrospective study}

Olgun Elicin, Lukas Anschuetz²\#, Beat Bojaxhiuㄹ, Daniel M. Aebersold ${ }^{1}$, Roland Giger ${ }^{2 *}$, Mohamed Shelan ${ }^{1 *}$

\section{\#,*: equal contribution}

${ }^{1}$ Department of Radiation Oncology, Inselspital, Bern University Hospital, University of Bern, Bern, Switzerland

${ }^{2}$ Department of Otorhinolaryngology, Head \& Neck Surgery, Bern University Hospital, University of Bern, Bern, Switzerland

Aims: Curative treatment of laryngeal squamous cell cancer can cause life-long morbidity and reduced quality of life. Our goal was to report the long-term functional outcome of various treatment modalities.

Methods: Charts of patients $(n=477)$ treated with curative intent for non-metastatic squamous cell carcinoma of the larynx between 1990 and 2014 were retrospectively reviewed. Time-to-event endpoints were calculated based on the date of diagnosis and compared with log-rank test. Uni-and multivariate analyses were done with Cox proportional hazards models.

Results: Radiotherapy (RT) with or without concomitant systemic treatment was the predominant $(75 \%)$ modality, whereas $25 \%$ underwent primary surgery. In the latter group, $72 \%$ required adjuvant treatment. Median follow-up was 51 months. The 5-year laryngectomy-free survival was $57 \%$ vs. $69 \%$ with primary surgery vs. RT, respectively $(p<0.01)$. In stage III-IVB, these rates were $26 \%$ vs. $47 \%$, respectively $(p<0.01)$. Tracheostomy at the time of last of follow-up was recorded in $39 \%$ and $21 \%$ following surgery and RT, respectively $(p<0.01)$. The risk of requiring a feeding tube at any time point was $52 \%$ and $30 \%$ in primary surgery and RT groups, respectively $(p<0.01)$. Multivariate analyses identified Stage III-IVB as the only adverse factor for permanent feeding-tube dependency (HR: 3.73, 95\% CI: $1.53-9.13 ; p<0.01$ ) and permanent tracheostomy (HR: $4.55,95 \%$ CI: $2.91-7.13 ; p<0.01$ ). Primary surgery (HR: $1.67,95 \%$ CI: $1.19-2.32 ; p<0.01$ ) and stage III-IVB (HR: $4.07,95 \%$ CI: $2.97-5.60 ; p<0.01$ ) remained as adverse factors for laryngectomy-free survival.

Conclusion: Preserving the laryngeal function in larynx cancer is challenging. The criteria for initial decision-making needs to be further refined. Posterwalk 
Definitive radiochemotherapy and salvage SBRT for stage IV anal cancer with lymphatic metastases-A case report

Thierry Panje

Radiation Oncology, Kantonsspital St. Gallen, St. Gallen, Switzerland

Introduction: Anal cancer is a rare gastrointestinal malignancy which presents at initial diagnosis with distant metastases in 10-20\%. Metastatic disease has a poor prognosis with 5-year survival rates below $20 \%$. Combined radiochemotherapy is the mainstay of treatment in locoregional disease whereas its use in stage IV disease is controversial and without level I evidence. Likewise, there is only very limited data on the benefit of metastasis-directed local therapy in anal cancer. We report a case of definitive radiochemotherapy at diagnosis and subsequent salvage stereotactic body radiotherapy in stage IV anal cancer. Patient history: A 56-y.o. female patient in good general health was diagnosed in 01/2015 with a squamous cell cancer of the anal canal with extensive lymphatic metastases up to the right common iliac artery, staged as cT3 cN3 cM1 (LYM). According to the multidisciplinary tumor board recommendation, she received an extended-field chemoradiation with 45 Gy with a sequential boost of 55.8 Gy to macroscopic disease and 2 cycles of concomitant chemotherapy with mitomycin $\mathrm{C}$ and 5-FU. Treatment was well tolerated and the patient remained disease-free for more than one and a half year. In November 2016, she developed retroperitoneal and mediastinal lymphatic metastases cranially from the irradiated volume. A palliative chemotherapy with 4 cycles of cisplatin and 5-FU was initiated. In March 2017, the patient underwent a consolidative stereotactic body radiotherapy of the macroscopic lymph node metastases in the retroperitoneum and mediastinum with $14 \times 3.5$ Gy without significant toxicity. Follow-up imaging showed an ongoing complete remission (last imaging in 04/2018). Conclusions: Our case demonstrates that extended-field radiochemotherapy and subsequent salvage SBRT can be effective in stage IV anal cancer with lymphatic metastases. Poster

\section{Comparison of contemporary staging systems for oropharynx cancer in a surgically treated multi-institutional swiss cohort}

Olgun Elicin ${ }^{1,2}$

${ }^{1}$ Department of Radiation Oncology, Inselspital, Bern University Hospital, University of Bern, Bern, Switzerland

${ }^{2}$ Swiss Oropharyngeal Cancer Working Group, Bern, Switzerland

Aims: Between the publication of the Union of International Cancer Control staging system (UICC) 7th and 8th editions, other staging algorithms for oropharyngeal squamous cell carcinoma (OPSCC) were proposed from Radiation Therapy Oncology Group (RTOG), MD Anderson Cancer Center (MDACC), and Yale University. Our aim was to compare the prognostic qualities of these 5 staging systems, test their applicability in terms of risk prognostication and investigate the rates of inter- and intra-stage migration among and within the clinical and pathological UICC 7th and 8th editions.

Methods: Rates of inter- and intra-stage migration among and within the clinical and pathological versions of the UICC 7th and 8th editions were investigated in a multi-institutional cohort of OPSCC cases $(n=338)$ treated with primary surgery. With C-statistics, the above-mentioned 5 staging systems were compared for overall and recurrence-free survival endpoints.

Results: In the UICC 8th ed, $19.4 \%$ post-operative up-staging and 45.4\% down-staging were observed in HPV- and HPV+ cases, respectively. For both endpoints, pathological UICC 8th edyielded the highest C-indexes in the entire cohort and in the HPV- cohort, whereas MDACC was superior for HPV+ OPSCC. RTOG was the most simple and holistic algorithm with a similar discriminatory power close to UICC 8th ed.
Conclusion: With each of their strengths and weaknesses, the UICC 8th ed, MDACC and RTOG systems offer moderate efficacy in similar magnitudes as staging algorithms for our patient cohort diagnosed with OPSCC and underwent surgical treatment. Notable discrepancy between clinical and pathological UICC 8th ed algorithms poses potential concerns in diagnosis, treatment, research and data management. Building complex models only to achieve a marginal benefit is a futile effort. Beyond an acceptable risk stratification accuracy, pursuing practical utility should be the primary goal of developing future algorithms of the UICC, as the internationally accepted authority for cancer staging. Poster

\section{Oncological outcome of loco-regionally advanced laryngeal cancer}

Olgun Elicin ${ }^{1 *}$, Mohamed Shelan ${ }^{1} \#$, Lukas Anschuetz²\#, Beat Bojaxhiu ${ }^{1}$, Daniel M. Aebersold ${ }^{1}$, Roland Giger ${ }^{2 *}$

\section{\#,*: equal contribution}

${ }^{1}$ Department of Radiation Oncology, Inselspital, Bern University Hospital, University of Bern, Bern, Switzerland

${ }^{2}$ Department of Otorhinolaryngology, Head \& Neck Surgery, Bern University Hospital, University of Bern, Bern, Switzerland

Aims: The optimal strategy to treat loco-regionally advanced laryngeal cancer remains to be defined. Our goal was to report a single institution experience in the treatment of loco-regionally advanced laryngeal cancer.

Methods: Charts of patients treated with curative intent for stage IIIIVB squamous cell carcinoma of the larynx between 1990 and 2014 were retrospectively reviewed. Day 0 was the date of diagnosis. Timeto-event endpoints were analyzed with Kaplan-Meier statistics.

Results: Of 154 patients, 57 (37\%) underwent primary surgery followed by adjuvant radiotherapy while the rest received primary radiotherapy with $(55 \%)$ or without the combination of a systemic therapy. Median follow-up was 50 months. The 5-year loco-regional control, recurrence-free (RFS), overall survival (OS) were $72 \%, 48 \%$ and $62 \%$, respectively. The OS between surgical and non-surgical primary modalities were similar in 5 years $(62 \% ; p=0.64)$. Patterns of recurrence and their treatment are given in Table 1. With a median follow-up of 16 months, the 2-year RFS and OS after salvage treatment were $43 \%$ and $48 \%$, respectively.

Conclusion: The results of our series are in line with the literature. The criteria to decide on treatment modalities in primary and loco-regionally recurrent larynx cancer need to be refined.

Table 1 Patterns of recurrence and treatment of recurrences

\begin{tabular}{ll}
\hline Variable & $N(\%)$ \\
\hline Recurrences (crude rate) & $50(32 \%)$ \\
Local & $27(54 \%)$ \\
Regional & $1(2 \%)$ \\
Loco-regional & $12(16 \%)$ \\
Distant metastasis & $18(16 \%)$ \\
Loco-regional and distant & $6(12 \%)$ \\
Treatment of recurrences & \\
Salvage surgery & $15(30 \%)$ \\
Salvage surgery + radiotherapy & $7(14 \%)$ \\
Palliative chemotherapy & $19(38 \%)$ \\
Palliative radiotherapy & $2(4 \%)$ \\
Best supportive care & $7(14 \%)$ \\
\hline
\end{tabular}




\section{Incidence of second primary cancers after radiotherapy combined with Platinum and/or Cetuximab in head and neck cancer patients}

Olgun Elicin ${ }^{1}$, Burim Sermaxhaj ${ }^{1}$, Beat Bojaxhiu ${ }^{1}$, Mohamed Shelan ${ }^{1}$, Roland Giger ${ }^{2}$, Daniel Rauch ${ }^{3}$, Daniel M. Aebersold

${ }^{1}$ Department of Radiation Oncology, Inselspital, Bern University Hospital and University of Bern, Bern, Switzerland

${ }^{2}$ Department of Otorhinolaryngology, Head and Neck Surgery, Inselspital, Bern University Hospital and University of Bern, Bern, Switzerland

${ }^{3}$ Department of Medical Oncology, Inselspital, Bern University

Hospital and University of Bern, Bern, Switzerland

Aims: The second primary cancer (SPC) incidence after treatment with platinum-based chemotherapy and Cetuximab in combination with radiotherapy is not reported. Our aim was to compare SPC risk following radiotherapy in combination with those agents for the treatment of head and neck squamous cell carcinoma (HNSCC).

Methods: The charts of 296 cases treated for loco-regionally advanced HNSCC between 2009 and 2015 were retrospectively reviewed for patient, tumor and procedural characteristics. All patients were planned to undergo radiotherapy either with platinum compounds (Group: Platinum) or monoclonal antibody Cetuximab (Group: Cetuximab). A third group of patients switched from platinum compounds to Cetuximab due to toxicity (Group: Switch). Treatment groups were evaluated for the incidence of SPC with log-rank test. Possible confounders were investigated with multivariate Cox's proportional hazards model. All tests were two-sided, and a $p<0.05$ was set to indicate statistical significance.

Results: Median follow-up was 36 months. Platinum, Cetuximab and Switch groups consisted of 158, 101 and 37 patients, respectively. Three-years overall survival in the whole cohort was $70 \%$. The rate of SPC between Platinum (9.2\%) and Cetuximab (11.5\%) groups were comparable, whereas the patients in Switch group (23.3\%) were exposed to a significantly higher incidence of SPC in 3 years $(p=0.01)$. The multivariate model indicated Switch as the only variable correlating with an increased risk for SPC.

Conclusion: Switch strategy may expose the patients to an increased risk of developing SPC. Further use of Switch should be strongly discouraged until robust pre-clinical and clinical data are available. Poster

\section{Toxicities with platinum and cetuximab concomitant to radiotherapy in head and neck cancer: An analysis with the TAME Method}

Olgun Elicin ${ }^{1}$, Burim Sermaxhaj ${ }^{1}$, Beat Bojaxhiu ${ }^{1}$, Mohamed Shelan ${ }^{1}$, Roland Giger ${ }^{2}$, Daniel Rauch ${ }^{3}$, Daniel M. Aebersold ${ }^{1}$

${ }^{1}$ Department of Radiation Oncology, Inselspital, Bern University Hospital and University of Bern, Bern, Switzerland

${ }^{2}$ Department of Otorhinolaryngology, Head and Neck Surgery, Inselspital, Bern University Hospital and University of Bern, Bern, Switzerland

${ }^{3}$ Department of Medical Oncology, Inselspital, Bern University Hospital and University of Bern, Bern, Switzerland

Aims: Acute toxicity (T), adverse late effects (A), mortality risk (M) and end results (E) methodology was developed by Trotti et al. [1]. Compared to conventional max-grade method, the cumulative toxicity profile can be better represented with TAME. Despite of that, TAME is still not established as a standard. Our goal was to analyze the toxicities ( $\mathrm{T}$ and $\mathrm{A}$ ) of concomitant radio-chemotherapy regimens in loco-regionally advanced head and neck squamous cell carcinoma (LAHSCC) using TAME.

Methods: The charts of 259 cases treated for LAHNSCC between 2009 and 2015 were reviewed for acute and late toxicity. All patients were treated with radiotherapy (RT) either with platinum compounds $(n=158)$ or cetuximab $(n=101)$. Toxicities were calculated with the T and A scores of TAME and compared with Wilcoxon rank test.

Results: Median follow-up was 36 months. Three-year loco-regional control was $88 \%$ in both treatment groups $(p=0.74)$. Mean T scores for Platinum and Cetuximab were $1.86 \pm 2.02$ and $2.75 \pm 2.59$, respectively $(p<0.01)$. Mean A scores for platinum and cetuximab were $0.26 \pm 0.65$ and $0.36 \pm 0.78$, respectively $(p=0.35)$.

Conclusion: Patients receiving RT and Cetuximab are more likely to suffer from grade $\geq 3$ acute toxicities compared to RT and Platinum combination. Potential selection bias needs to be further investigated.

\section{References}

1. Trotti A et al (2007) TAME: development of a new method for summarising adverse events of cancer treatment by the Radiation Therapy Oncology Group. Lancet Oncol 8(7):613-624

\section{A cost-effectiveness analysis of consolidative local therapy in oligometastatic non-squamous non-small cell lung cancer}

CM Panje ${ }^{1}$, KJ Dedes², K Matter-Walstra ${ }^{3,4}$, M Schwenkglenks ${ }^{4}$, $\mathrm{O}_{\text {Gautschi }}^{5}$, M Siano ${ }^{6}$, DM Aebersold ${ }^{7,8}$, L Plasswilm $^{1,8}$,

J E Lupatsch ${ }^{3,4}$ for the Swiss Group for Clinical Cancer Research (SAKK)

${ }^{1}$ Department of Radiation Oncology, Cantonal Hospital St. Gallen, St. Gallen, Switzerland; ${ }^{2}$ Department of Gynecology, University Hospital Zurich, Zurich, Switzerland; ${ }^{3}$ Swiss Group for Clinical Cancer Research Coordinating Centre, Bern, Bern, Switzerland; ${ }^{4}$ Institute of Pharmaceutical Medicine, University of Basel, Basel, Switzerland; ${ }^{5}$ Medical Oncology, Department of Internal Medicine, Cantonal Hospital Lucerne, Lucerne, Switzerland; ${ }^{6}$ Department of Medical Oncology, Cantonal Hospital St. Gallen, St. Gallen, Switzerland; ${ }^{7}$ Department of Radiation Oncology, University Hospital Bern, Bern, Switzerland; ${ }^{8}$ University of Bern, Bern, Switzerland

Background: Treatment costs in metastatic non-small cell lung cancer (NSCLC) are a matter of ongoing debate, particularly for novel systemic therapies. More recently, local therapies (LT) such as radiotherapy have been suggested as additional treatment in oligo-metastatic NSCLC demonstrating an improved progression-free survival (PFS) in a phase II trial compared to maintenance chemotherapy (MC) alone. The aim of this analysis was to assess the cost-effectiveness of local therapies in oligo-metastatic NSCLC.

Methods: We constructed a Markov model comparing the cost-effectiveness of LT versus MC for oligo-metastatic NSCLC from the Swiss healthcare payer's perspective. Treatment specifications and PFS were primarily based on the phase II trial. Overall survival (OS) was inferred from a recent phase III trial. Primary outcome was the incremental cost-effectiveness-ratio (ICER, costs in Swiss Francs (CHF) per quality-adjusted life-year (QALY) gained).

Results: LT was cost-effective with a gain of 0.24 QALYs at an additional cost of CHF 9,641, resulting in an ICER of 40,972/QALY gained. Probabilistic sensitivity analyses demonstrated that LT was dominant or cost-effective at a willingness-to-pay threshold of CHF 100,000 per QALY in $61.7 \%$ of the simulations.

Conclusions: LT may be cost-effective for selected patients with oligo-metastatic NSCLC responding to first-line systemic therapy. Poster

\section{Impact of multidisciplinary team management in a rare setting} of head and neck cancer patients

F. Martucci, MC. Valli, C. Azinwi, D. Bosetti, S. Cima, M. I. Donegani, I. Maddalena, GA Pesce, A. Richetti

Radiation Oncology, Oncology Institute of Southern Switzerland, Bellinzona, Switzerland 
Objective: To investigate how to handle patients (pts) with head and neck squamous cell carcinoma (SCC) of unknown primary within a multidisciplinary team (MDT).

Matherials and methods: In our institution, every 3 weeks, we discuss the cases of head and neck cancer pts within a MDT before any treatment. From January 2011 to December 2017 nearly 350 pts were discussed; $10(2.8 \%)$ were affected by SCC of unknown primary. Head and neck SCC (HNSCC) of unknown primary is a relatively rare entity ( $1-3 \%$ of new cases) in the head and neck region, defined as the presence of SCC in one or more lymph nodes of the region without an identifiable primary tumor. The management of this population is very difficult due to the involvement of more specialities. All our patients, after a physical examination, were staged with PET/CT and panendoscopy, with or without tonsillectomy, to confirm the absence of primary tumour. Of 10 pts discussed, 3 were in stage III and 7 in stage IVA/B; 6 pts were HPV positive. Seven pts underwent neck surgery, all pts received radiotherapy on the potential $\mathrm{HN}$ primary tumor site and mono/ bilateral neck nodes according to international guidelines. Three pts were treated with exclusive concurrent radio-chemotherapy.

Results: With a median follow up of 27 months all 10 pts are alive. Two pts experienced the appearance of the primary tumor: one was diagnosed with a skin scalp tumor after 60 months, the other with an oropharyngeal tumor, 50 months after oncologic treatments. In both cases the primary tumor had the same characteristics of the previous nodal histology. We registered low grade radiation toxicity: dermatitis and mucositis, maximum grade 2 , according to the RTOG scale.

Conclusions: Our experience confirms, as reported in literature, the crucial role of a multidisciplinary dedicated team to define the workflow of staging, treatments and modalities of follow-up, in such an uncommon tumor. Poster

\section{Urethra-sparing prostate cancer SBRT: 18-months results from} a once-a-week vs. every-other-day randomized phase II trial

Giorgio Lamanna

\section{Radiation Oncology, HUG, Genève, Geneva, Switzerland}

Purpose: To present the 18-month results from a phase II randomized trial of short vs. protracted urethra-sparing SBRT for localized prostate cancer (PCa).

Materials/Methods: From 08/2012 through 12/2015, 170 patients (pts) from 9 institutions with cT1c-3aN0M0 PCa and a risk of nodal involvement $\leq 20 \%$ were randomized according to two different o schedules: either 9 days (arm A, 84 pts), or 28 days, once-a-week, the same week-day ( $\mathrm{arm} \mathrm{B}, 86 \mathrm{pts}$ ). The prescribed dose was $36.25 \mathrm{~Gy}$ (7.25 Gy x 5) in both arms, with a urethra sparing of 32.5 Gy $(6.5 \mathrm{~Gy} x$ 5). SBRT was delivered with VMAT or IMRT using Novalis linacs and the ExacTrac IGRT technology. Genitourinary (GU) and gastrointestinal (GI) toxicity (CTCAE v4.03 grading scale), IPSS, and QoL scores (EORTC QLQ-PR25) were assessed at baseline, at the 5th fraction (5 fx), 12th weeks (W12) and every 6 months since SBRT.

Results: 82 and 83 pts, respectively, from arms A and B, were retained for this analysis (5 out of 170 randomized pts were never treated). A toxicity stopping rule was activated during the first 3-months of pts' FU though was never triggered. Grade- 2 GU toxicity rates at baseline, $5 \mathrm{fx}$, and $\mathrm{W} 12$ were $2 \%, 17 \%$, and $11 \%$ vs. $5 \%, 19 \%$ and $6 \%$ for pts in arms A and B, respectively (mainly moderate irritative and voiding symptoms). Only one case of obstructive grade- $3 \mathrm{GU}$ toxicity was observed at W12 in arm B. Two cases of grade-2 GI toxicity (2.5\%) were observed at $5 \mathrm{fx}$ in $\operatorname{arm} \mathrm{A}$.

Grade-2 GU toxicity decreased up to a $4 \%$ rate in both arms at the last FU. One case of grade- 3 GU toxicity was observed in arm A. The incidence of grade- 2 GI toxicity was below $3 \%$ in both arms. Concerning IPSS, the rate of pts satisfied at baseline and 18-months were $80 \%$ and $90 \%$ for arm A and 77 and $88 \%$ for arm B, respectively. No changes in EORTC QLQ-PR25 scores were observed in both arms during the first 18 months. Median PSA decreased from 8.3 and $7 \mathrm{ng} /$ $\mathrm{ml}$ at baseline to 0.53 and $0.46 \mathrm{ng} / \mathrm{ml}$ at month 18 for arms A and B, respectively.

Conclusions: 18-months results of this trial demonstrate for both study arms the good tolerance and toxicity profile of both treatment arms with minimal impact on QoL. Oral presentation

\section{Definitive radiochemotherapy and salvage SBRT for stage IV anal cancer with lymphatic metastases-A case report}

T. Panje ${ }^{1}$, C. Panje ${ }^{1}$, D. Brügge ${ }^{1}$, U. Güller ${ }^{2}$, L. Plasswilm ${ }^{1}$

${ }^{1}$ Department of Radiation Oncology and ${ }^{2}$ Department of Medical Oncology, Kantonsspital St. Gallen, St. Gallen, Switzerland

Introduction: Anal cancer is a rare gastrointestinal malignancy which presents at initial diagnosis with distant metastases in 1--20\%. Metastatic disease has a poor prognosis with 5-year survival rates below $20 \%$. Combined radiochemotherapy is the mainstay of treatment in locoregional disease whereas its use in stage IV disease is controversial and without level I evidence. Likewise, there is only very limited data on the benefit of metastasis-directed local therapy in anal cancer. We report a case of definitive radiochemotherapy at diagnosis and subsequent salvage stereotactic body radiotherapy in stage IV anal cancer. Patient history: A 56-y.o. female patient in good general health was diagnosed in 01/2015 with a squamous cell cancer of the anal canal with extensive lymphatic metastases up to the right common iliac artery, staged as cT3 cN3 cM1 (LYM). According to the multidisciplinary tumor board recommendation, she received an extended-field chemoradiation with 45 Gy with a sequential boost of 55.8 Gy to macroscopic disease and 2 cycles of concomitant chemotherapy with mitomycin $\mathrm{C}$ and 5-FU. Treatment was well tolerated and the patient remained disease-free for more than one and a half year. In November 2016, she developed retroperitoneal and mediastinal lymphatic metastases cranially from the irradiated volume. A palliative chemotherapy with 4 cycles of cisplatin and 5-FU was initiated. In March 2017, the patient underwent a consolidative stereotactic body radiotherapy of the macroscopic lymph node metastases in the retroperitoneum and mediastinum with $14 \times 3.5 \mathrm{~Gy}$ without significant toxicity. Follow-up imaging showed an ongoing complete remission (last imaging in 04/2018). Conclusions: Our case demonstrates that extended-field radiochemotherapy and subsequent salvage SBRT can be effective in stage IV anal cancer with lymphatic metastases. Poster

\section{Histopathological changes after RT and Re-RT of spine metastases with SBRT}

Robert Foerster

Department of Radiation Oncology, University Hospital Zurich, Zurich, Switzerland

Aims: Stereotactic body radiotherapy (SBRT) of the spine provides superior tumor control, but vertebral compression fractures are common and the pathophysiological process is not well understood. Data on histopathological changes after SBRT are scarce and patients may be at an increased risk of osteonecrosis. Thus, we sought to investigate surgical specimens after salvage SBRT (sSBRT) and primary SBRT (pSBRT). Methods: 704 patients underwent sSBRT $(69.6 \%)$ or pSBRT $(30.4 \%)$ for spinal bone metastases between 2006 and 2012. 30 patients received salvage surgery. 23 histopathological reports were available. Clinical and histopathological findings of both groups were analyzed and compared.

Results: Mean time to surgery after sSBRT/pSBRT: 8.3/10.3 months $(p=.64)$. Reasons for salvage surgery: pain (sSBRT/pSBRT: $12.5 \% / 71.4 \%, p=.25$ ), fractures (sSBRT/pSBRT: $37.5 \% / 28.6 \%, p=.68$ ), neurological symptoms (sSBRT/pSBRT: $68.8 \% / 42.9 \%, p=.24)$. Radiological tumor progression after sSBRT/pSBRT: $71.4 \% / 42.9 \%(p=.20)$. 
Histopathological findings were similar. Most specimens: viable/proliferative tumor (sSBRT/pSBRT: $62.5 \% / 71.4 \%, p=.68$ and $56.3 \% / 57.1 \%$, $p=.97$ ). Few specimens: soft tissue necrosis (sSBRT/pSBRT: 20\%/28.6\%, $p=.66$ ), osteonecrosis (sSBRT/pSBRT: $14.3 \% / 16.7 \%$, $p=.89$ ), bone marrow fibrosis (sSBRT/pSBRT: $42.9 \% / 33.3 \%, p=.69$ ). Tumor bed necrosis was more common after sSBRT $(81.3 \% / 42.9 \%$, $p=.066)$. Radiological tumor progression correlated with viable/proliferative tumor $(p=.03 / p=.006)$ and tumor bed necrosis $(p=.03)$. Fractures were increased with bone marrow fibrosis $(p=.07)$, but not with osteonecrosis $(p=.53)$ or soft tissue necrosis $(p=.19)$. Neurological symptoms were common with radiological tumor progression $(p=.07)$, but not with fractures $(p=.18)$. Pain did not correlate with tumor progression $(p=.72)$ or pathological fractures $(p=.19)$.

Conclusion: Histopathological changes were similar after sSBRT and pSBRT. Neurological symptoms were chiefly attributable to tumor progression. Pathological fractures were neither associated with osteonecrosis nor tumor progression. Evidence of viable tumor in most specimens calls for an aggressive treatment with sSBRT and pSBRT. Poster

\section{Multi-institutional treatment of patients with cervical cancer in Switzerland}

\section{Vlaskou BE, Tsikkinis A, Cihoric N, Aebersold DM, Lössl K}

Radiation Oncology, Inselspital Bern, Bern, Switzerland

Objective: to evaluate the outcome, toxicity and patterns of treatment failure in patients (pts) treated with image guided external beam radio(chemo)therapy (IG-EBRcT) and centralized IG high dose rate brachytherapy (IG-HDR).

Materials and methods: From 01/2012 to 06/2017, 96 (100\%) pts with cervical cancer (FIGO IA2-IVA) were treated with IG-EBRcT in 10 Swiss institutions. $74 \%$ of pts were diagnosed with positive lymph nodes, $21 \%$ after complete or sentinel-lymphadenectomy and $53 \%$ via ${ }^{18} \mathrm{FDG}-\mathrm{PET} / \mathrm{CT}$. $81 \%$ of pts were diagnosed with tumors larger than $4 \mathrm{~cm}$ in diameter. $82 \%$ of the tumors were squamous cell carcinomas, $10 \%$ adenocarcinomas and $7 \%$ other histologies. All pts were treated with IG-EBRcT. A dose of 50.4 Gy was prescribed to the elective pelvic nodal volume. Primary tumors $<4 \mathrm{~cm}$ in diameter received an additional boost of $5.4 \mathrm{~Gy}$, primary tumors $>4 \mathrm{~cm}$ in diameter received a boost of 9 Gy. Pts with positive lymph nodes detected with ${ }^{18}$ FDG$\mathrm{PET} / \mathrm{CT}$ received a simultaneous integrated boost to a total dose of 64 Gy. Chemotherapy was given according to institutional standards. After completion of IG-EBRcT all pts were referred to Bern University Hospital for IG-HDR treatment $(4 \times 6 \mathrm{~Gy})$. Median EQD2 dose for the primary tumor was $81.6 \mathrm{~Gy}$ (62.6--3.2 Gy). Adverse events were coded according to CTCAE v 4.0.

Results: Survival data were available for 91 pts. During a median follow-up of 22 months (2-77 months) 28 (31\%) pts developed disease relapse. $12(13 \%)$ pts had loco-regional relapse, $7(8 \%)$ local and $5(5 \%)$ nodal relapse. $22(24 \%)$ developed systemic metastases. $13(14 \%)$ pts died, $9(10 \%)$ from cervical cancer. Median survival time was not reached. In total 89 acute adverse events (AE) were recorded $(86 \mathrm{Gr}$. $1-2 ; 3$ Gr. 3). Data on late AE were available for $83 \%$ of pts. 68 late AE were recorded ( 37 Gr. 1; 26 Gr. 2; 5 Gr. 3).

Conclusion: Satisfactory treatment outcomes were achieved while treatment-related toxicities were kept reasonably low. Poster

\section{A case of suspected breast cancer metastasis to brachial plexus NOT detected by magnetic resonance neurography or any other imaging}

\section{T. Mader, D. Brügge, T. Hundsberger}

Radiooncology, Kantonsspital St. Gallen, St. Gallen, Switzerland

Aim: Malignant brachial plexopathy is a rare complication of metastatic spread in breast cancer. As the metastatic origin is frequently dif- ficult to detect, the neurologic symptoms can persist a long time until the cause is found.

In this case report we present a patient with symptomatic brachial plexopathy as first and only clinical manifestation of a metastatic spread in a known mammary carcinoma.

Methods: The patient presented in our outpatient clinic with a progressive sensory and motoric deficit of the right upper extremity and pain of the right shoulder and elbow joint over the last 1-2 years. Magnetic resonance neurography and PET-CT scans showed no suspicious lesions in the brachial plexus, the spine or the shoulder joint. However the neurological examination was highly suspicious of brachial plexus affection. Together with the clinical record of the breast cancer and a asymptomatic metastatic lesion in the right superior lung lobe the neurological sings led to the postulation of a metastatic cause of the plexopathy. We applied a percutaneous radiotherapy to the right brachial plexus over its whole course with a total dose of 54 Gy (Gray). A daily dose from 1.8 Gy over 30 fractions, 1 fraction per day, using 6MV photons was administered.

Results: The radiotherapy was well tolerated by the highly motivated patient. A temporary grade II laryngitis subsided at the end of the radiotherapy and other cutaneous or neurological radiogenic side-effects were not seen.

The neurological symptoms of the right arm improved with a significant increase in strength in both the forearm and the hand. The sensory impairments, especially the feeling of cold and the numbness diminished. The electrifying and stabbing pain was also improved but still substantially present.

Conclusion: Radiation is highly effective in malignant brachial plexopathy when clinical and neurological symptoms lead to a hypothetical diagnosis. Poster

\section{Local control following highly conformal SIB-IMRT for T1/2 glottic cancer-update}

Studer G, Glanzmann C, Janssen S, Iff M, Streller T, Tanadini-Lang S, Guckenberger M, Riesterer O

${ }^{1}$ Radiation Oncology, Cantonal Hospital Lucerne, Lucerne, Switzerland

${ }^{2}$ University Hospital Zurich, Zurich, Switzerland

Aims: To update our previously shown effectiveness data of partial laryngeal- and/or carotid artery sparing SIB-IMRT in low to intermediate risk tumors.

Results: Between 12/2004 and 10/2015, 67 consecutive patients with cT1/2 (35/32) cN0 glottic carcinoma were definitively irradiated using conformal SIB-IMRT techniques at the department of Radiation Oncology, University Hospital Zurich. In 59/67 patients the anterior commissure was involved. Treatment schedules included $5 \times 2.0 \mathrm{~Gy} / \mathrm{w}$ to $70 \mathrm{~Gy}, 6 \times 2.0 \mathrm{~Gy} / \mathrm{w}$ to 68 or $5 \times 2.25 \mathrm{~Gy} / \mathrm{w}$ to $63 \mathrm{~Gy}$ for PTV1. Mean follow up (FU) was 48 months (1.4-113), mean/median age of patients was $66.7 / 67$ years (38-87). We observed 4/67 local failures; 3/4 patients underwent successful salvage laryngectomy, 1 patient was successfully lasered. The 3-year local and locoregional disease free survival rates were $93.8 \% / 94 \%$, the overall survival rate $90 \%$, respectively. One cT2 patient with no signs of disease unfortunately died 10 months post IMRT completion due to unexpected massive laryngeal edema with consecutive unsuccessful intubation. Another cT2 patient developed distant disease. The mean dose to the ipsilateral contoured segment of the carotid artery could be kept $<30$ Gy. Partial larynx sparing was impossible in $15 / 67$ patients due to the tumor extension.

Conclusion: Presented data based on longer FU and bigger sample size confirm previous findings on small volume conformal SIB-IMRT being safe and effective in T1/2 glottic cancer. Local recurrence localisation and comparison with the literature will be presented. Poster 


\section{Biology track}

\section{Radiation induce liver disease mouse model; A promising step toward understanding of normal liver tissue toxicity}

N. Melin ${ }^{1}$, D. Sánchez 1 , B. Petit ${ }^{2}$, E. Herman ${ }^{1}$, Y. Zimmer ${ }^{1}$,

D. Candinas ${ }^{1}$, M. C. Vozenin ${ }^{2}$, D. Aebersold ${ }^{1}$, D. Stroka ${ }^{1}$

${ }^{1}$ Department for BioMedical Research, University of Bern, Bern, Switzerland

${ }^{2}$ Centre Hospitalier Universitaire Vaudois, Lausanne, Switzerland

Aims: Radiation of liver tumors has recently shown promising results in clinics, but it applicability is limited by the life-threatening condition resulting from normal liver toxicity. The radiation induce liver disease (RILD) develops few weeks after irradiation, and characterized by the development of sinusoid obstruction syndrome. Although the risk of RILD can be estimated, the underlying mechanism is unknown. Here we describe our RILD mouse model and subsequent finding on it development.

Methods: Based on CT images obtained using a liver contrasting agent, we irradiated a $5 \mathrm{~mm}$ diameter liver target with 50 Gy using image guided irradiation. Sample collection were realized at 1day, 6day, 6 week and 20week post irradiation. Histological evaluation of thr liver were realized using eosin hematoxylin, Masson's trichrome, Sirius red and Evans blue staining. Transcriptomic analysis was perused using two samples of RNA per liver (from the target area and not irradiated area; Paired analysis).

Results: Irradiated mice did not show histological change at day 1 and day6. At 6 weeks centrizonal sinusoid were obstructed by collagen fiber and an increase number of Evan's blue positive ceroid containing macrophage where observed in the target area. At 20 weeks the observation where amplified. Transcriptomic analysis revealed very different profile at the different time points. After the initial injury, a large number of repair mechanism are ongoing at day 1 . At day 6 a very few process where find different between high and low irradiated samples. Week 6 and 20 showed an increasing number of process ongoing and among them ECM modulator and immune response processes as expected from the histology. Based on the course of the disease, we hypothesis that the key trigger of RILD is the unresolved mitochondrial injury that we could observe at day6.

Conclusion: Further characterization RILD marker should allow further evaluation of prophylactic and therapeutic interventions. Posterwalk

\section{Radiosensitization strategy based on DNA-PK inhibition in head and neck cancer: Emphasis on human papilloma virus (HPV)}

Selina Moara Roth ${ }^{1}$, Daniel Matthias Aebersold ${ }^{1}$, Yitzhak Zimmer ${ }^{1}$, Michaela Medová ${ }^{1}$

${ }^{1}$ Department for BioMedical Research, Radiation Oncology, Inselspital, Bern University Hospital, and University of Bern, Bern, Switzerland

Aims: Head and neck cancer is the sixth most common non-skin cancer worldwide, leading to more than 350'000 deaths per year. Despite the use of improved organ-sparing treatments consisting of surgery and chemoradiation, loco-regional or distant relapses after completion of definitive therapy occur in up to $50 \%$ of all patients. Therefore, identification of new strategies to improve outcome is needed. In this study, we aim on assessing potential sensitization of tumors to ionizing radiation (IR) by inhibiting DNA-PK, a DNA-dependent nuclear serine/ threonine kinase critical for the repair of DNA-double strand breaks (DSB) on a panel of head and neck squamous cell carcinoma (HNSCC) cell lines.
Methods: In order to sensitize HNSCC cell lines to irradiation we are using the DNA-PK inhibitor M3814, currently being investigated in a $\mathrm{PhI}$ clinical study. Specifically, we examine the impact of irradiation, DNA-PK inhibition and the combination of these two treatments on proliferation, survival and cell death. To study whether differences in treatment outcome are due to the genetic background, the cell lines are divided into HPV+/p53wt, HPV-/p53wt, HPV-/p53mutated groups. Results: Our results show that the drug alone does not cause any cytotoxic effects, but when combined with IR, an effect on viability, proliferation and survival can be observed. By anticipating the DSB repair through DNA-PK inhibition, we can attain an enhancement of the effect of IR on cell viability. Interestingly, although proliferation is reduced in all cell lines, only HPV+/p53wt and HPV-/p53mutated cells undergo a G2 cell cycle arrest and apoptosis.

Conclusion: We have corroborated the radio-enhancing effect of DNA-PK inhibition on proliferation, survival and viability of HNSCC cell lines independently of their HPV or p53 status. Ultimately, apoptosis is induced in HPV+/p53wt and HPV-/p53mutated cells, and apoptosis-independent cell death can be observed in HPV-/p53wt cells. Poster

\section{DNA-PK regulates the radiosensitivity of MET-addicted cancer cell lines via a novel MET phosphosite}

Jonas P. Koch ${ }^{1}$, Selina M. Roth ${ }^{1}$, Aurélie Quintin ${ }^{1}$, Jacopo Gavini ${ }^{2}$, Deborah M. Stroka ${ }^{2}$, Daniel M. Aebersold ${ }^{1}$, Yitzhak Zimmer ${ }^{1}$, Michaela Medová

${ }^{1}$ Department for BioMedical Research, Radiation Oncology, Inselspital, Bern University Hospital, and University of Bern, Bern, Switzerland

${ }^{2}$ Department for BioMedical Research, Visceral Surgery, Inselspital, Bern University Hospital, and University of Bern, Bern, Switzerland

Aims: MET, the receptor tyrosine kinase for hepatocyte growth factor, is a proto-oncogene mainly expressed in epithelial cells and involved in development, homeostasis and tissue regeneration. Deregulation of MET signaling has been reported in numerous cancers, leading to a broad interest in MET targeting for therapy. Additionally, there is emerging evidence that MET signaling extends to the DNA damage response (DDR) machinery and protects tumors from DNA-damaging agents. This study aims to investigate the function of a novel MET phosphosite in the context of radioresistance.

Methods: In vitro and in vivo assays were used to assess the radiosensitivity of human cancer cell lines overexpressing constitutively active MET and transformed mouse fibroblast cells ectopically expressing wild type or activating MET-mutated variants combined with a phosphodeficient Ser to Ala mutation.

Results: A study of post-translational changes in a MET-addicted cancer cell line upon MET inhibition and ionizing radiation revealed a yet unreported phosphorylation site on MET that is part of a consensus motif recognized by master DDR kinases. Our results show that this site can be phosphorylated by DNA-PK and that its phosphorylation fluctuates in response to MET inhibition. MET-addicted transformed cells expressing the phosphodeficient (Ser to Ala mutation) form of active MET prematurely evade from irradiation-induced cell cycle arrest, leading to abnormal mitosis and lower proliferation, rendering them more radiosensitive than their nonmutated counterpart.

Conclusions: We characterize a novel MET phosphosite that bears an important function for radiosensitivity and oncogenicity of MET-addicted cancer cell lines, providing new insights into the crosstalk linking MET and the DDR. Oral presentation 
Table 1

\begin{tabular}{lll}
\hline KEGG Pathways & $p$-value & No. of target genes \\
\hline p53 signaling pathway (hsa04115) & 0.001366 & 26 \\
$\begin{array}{l}\text { Pathways in cancer (hsa05200) } \\
\text { Transcriptional misregulation in } \\
\text { cancer (hsa05202) }\end{array}$ & 0.003607 & 91 \\
$\begin{array}{l}\text { PI3K-Akt signaling pathway } \\
\text { (hsa04151) }\end{array}$ & 0.006312 & 47 \\
$\begin{array}{l}\text { MAPK signaling pathway } \\
\text { (hsa04010) }\end{array}$ & 0.032828 & 56 \\
Prostate cancer (hsa05215) & 0.032828 & 27 \\
\hline
\end{tabular}

\section{Profiling of exosomal miRNA in non-metastatic prostate cancer after radiation therapy}

Mohamed Shelan ${ }^{1} \#$, Bijaya Malla ${ }^{1} \#$, Timo Nannen ${ }^{1}$, Alan Dal Pra ${ }^{1}$, Daniel M. Aebersold

\author{
\#Equal contribution \\ ${ }^{1}$ Department of Radiation Oncology, Bern University Hospital, \\ University of Bern, Bern, Switzerland
}

Aims: Biomarkers for treatment response and relapse after radiotherapy (RT) in non-metastatic prostate cancer (PC) are not well established. Exosomal microRNAs (exomiRs) are key regulators of cellular pathways influenced by RT, indicating its potential as liquid biopsy biomarkers. We aimed to investigate RT induced changes in exomiRs in pilot sample from ongoing prospective study.

Methods: We profiled exomiRs by RNA sequencing (Exiqon, Denmark) in patients receiving RT for primary PC Group A; $(n=6)$, biochemically relapsed after prostatectomy (Group B; $n=3$ ), and healthy controls $(n=3)$. To characterize RT induced expression in exomiRs, we compared paired samples collected pre- and 28 days post-RT. We used edgeR Bioconductor package to perform differential expression analysis using fold change $\geq 1.2$ at FDR $<0.10$. The putative target genes of exomiRs and respective pathways were analyzed using DIANA-mirPath v3.

Results: We identified four exomiRs differentially expressed after RT in Group A (miR-365a-3p, miR-27b-5p, miR-365b-3p, and miR-652$3 p$ ), and one in Group B (let-7f-5p). Compared to pre-RT samples, expression of miR-652-3p, and let-7f-5p decreased while others were upregulated. The KEGG pathway analysis using experimentally verified targets from TarBase v6 generated 35 candidate pathways, some of those relevant to PC are listed below (Table 1).

Conclusion: In this explorative study, we were able to identify candidate exomiRs after RT in non-metastatic PC, which sought for investigation in larger cohorts. Posterwalk

\section{Unravelling the molecular response to dose-rate and delivery time of ionizing radiation}

Dorota Dudka ${ }^{1}$, Ning Chang ${ }^{1}$, Andreas Joosten ${ }^{2}$, Jianhua Feng ${ }^{1}$, Michaela Medová ${ }^{1}$, Yitzhak Zimmer ${ }^{1}$ and Kathrin Zaugg ${ }^{1,3}$

${ }^{1}$ Department for BioMedical Research, Radiation Oncology, Inselspital, Bern University Hospital, and University of Bern, Bern, Switzerland

${ }^{2}$ Division of Medical Radiation Physics and Department of Radiation Oncology, Inselspital, Bern University Hospital, and University of Bern, Bern, Switzerland

${ }^{3}$ Department of Radiation Oncology, Stadtspital Triemli, Zurich, Switzerland

Aim: There is increasing evidence that modulation of dose-rate and delivery time can affect tumor cell survival after ionizing radiation (IR).
The clinical significance and the underlying molecular mechanisms are still unclear. In this study we hypothesize that decreasing delivery time or increasing dose-rate in radiation therapy significantly changes molecular response, leading to a more pronounced activation of cell death signaling pathways.

Methods: Experiments were performed using HCT116 and HEK293 cells growing as spheroids (3D culture) or monolayers (2D). We used flow cytometry analysis (Annexin V and PI staining) to compare cell death. In addition, the formation of DNA double strand breaks $(\gamma \mathrm{H} 2 \mathrm{AX})$, activation of DNA damage pathways (phospho p53), cell cycle and proliferation in response to IR with two different delivery times: 43 min 45s (low dose rate) and 2 min $55 \mathrm{~s}$ (high dose rate) was examined.

Results: Results showed, independently of delivery time, a decrease in cell viability in 2D compared to 3D culture using dose of $10 \mathrm{~Gy}$ or 20 Gy for HEK293 and HCT116 cell lines respectively. An increase in $\gamma \mathrm{H} 2 \mathrm{AX}$ expression was observed after IR however, we noticed no significant change between the two dose rate. Similarly, no significant difference in $\mathrm{p} 53$ activation between the two different dose-rates of IR was detected. We observed different radiation sensitivity of 2D and 3D culture in respect to cell cycle redistribution and proliferation after IR, but independently to delivery time.

Conclusions: 3D cell culture is more resistant to IR treatment when compared to $2 \mathrm{D}$ cell culture. Further investigations are needed to clarify the molecular mechanisms of response to different delivery time. Poster

\section{Targeting MET modulates global metabolic pathways and induces dNTPs depletion-associated DNA damage}

Michaela Poliaková ${ }^{1}$, Nicola Zamboni ${ }^{2}$, Jonas P. Koch ${ }^{1}$, Daniel M. Aebersold ${ }^{1}$, Yitzhak Zimmer ${ }^{1}$, Michaela Medová ${ }^{1}$

${ }^{1}$ Department for BioMedical Research, Radiation Oncology, Inselspital, Bern University Hospital, and University of Bern, Bern, Switzerland

${ }^{2}$ Institute of Molecular Systems Biology, ETH Zürich, Zurich, Switzerland

Aims: Overexpression of the MET receptor tyrosine kinase observed in various tumors is often associated with poor prognosis and furthermore, HGF/MET signaling confers resistance to cancer treatments by protecting tumors from DNA-damaging agents. In order to gain insights into the link between MET receptor and cellular DNA damage response, we conducted a metabolome-wide profiling to investigate the impact of MET inhibition (METi) on cellular metabolism in METi-responsive in vitro cancer models.

Methods: Findings of MS-based non-targeted metabolomics analysis were further studied by cell-based assays, Western blotting, qPCR, fluorescent microscopy and transcriptomics.

Results: METi caused significant alterations in energy metabolism, tricarboxylic acid cycle and amino acid metabolism in MET-addicted models. Interestingly, 5'-Phosphoribosyl- $N$-formylglycinamide, a metabolite involved in the de novo purine synthesis pathway, was consistently decreased in all MET-addicted cellular systems upon METi. METi-induced downregulation of critical purine synthesis enzymes resulted in depletion of important DNA building blocks, the dNTPs. Transient E2F1 overexpression prevented the drop of dNTPs and the induction of DNA damage that occurs in MET-addicted cells after administration of the MET inhibitor.

Conclusion: We report for the first time that MET targeting modulates global metabolomics pathways including de novo purine synthesis. Moreover, DNA damage inflicted by METi in MET-addicted models results from E2F1-regulated depletion of dNTPs. Poster 


\section{Tumor reoxygenation and image-guided SBRT for the} treatment of hypoxic tumors

I. Grgic ${ }^{1}$, N. Borgeaud ${ }^{2}$, A. Gupta ${ }^{2}$, M. Guckenberger ${ }^{1}$, R. Graf ${ }^{2}$, M. Pruschy

${ }^{1}$ Department of Radiation Oncology, University Hospital Zürich, Zurich, Switzerland

${ }^{2}$ Department of Surgery, University Hospital Zürich, Zurich, Switzerland

Aims: Hypoxic tumor cells are resistant to ionizing radiation (IR). Hence, reoxygenation of the hypoxic tumor fraction by a combined treatment modality with a pharmaceutical agent is of high interest. Here we investigated the combined treatment modality of the novel anti-hypoxia compound myo-inositol trispyrophosphate (ITPP) in combination with IR for the treatment of murine colorectal liver metastases.

Methods: ITPP was developed as an effector of hemoglobin lowering the oxygen/hemoglobin affinity thereby resulting in an enhanced release of oxygen e.g. in hypoxic tumors. Tumor growth delay was determined on treatment with ITPP and a single high dose fraction of IR in subcutaneous tumor models (FaDu, A549, MC38) and an orthotopic colorectal liver metastases tumor model. Tumor volumes were probed by caliper measurements (subcutaneous tumors) and by serial MRI (orthotopic tumors). Tumor detection and irradiation were performed by contrast-enhanced CT and a small animal radiotherapy platform.

Results: Using our in vivo bioimaging approach, we confirmed increased $\mathrm{pO}_{2}$ starting $2 \mathrm{~h}$ after ITPP application. Interestingly, ITPP alone did not affect the growth of tumor xenografts but significantly sensitized the tumor to IR in all tested tumor models. Immunohistochemical analysis of $\gamma \mathrm{H} 2 \mathrm{AX}$ foci demonstrated increased IR-induced DNA damage within hypoxic tumor regions after combined treatment with ITPP as compared to IR alone. With IR-dose-titration experiments to orthotopic colorectal liver metastasis, limiting single-doses of IR with tolerable toxicities could be defined, which are currently combined with ITPP as part of efficacy-oriented experiments using image-guided radiotherapy.

Conclusion: Here we demonstrated that the combined treatment modality of ITPP and IR results in a supra-additive tumor growth delay in multiple tumor models. Overall, our results support the strong rationale to combine ITPP with hypofractionated radiotherapy for hypoxic tumors. Oral presentation

\section{Physics track}

\section{A risk assessment method for lymphatic metastatic spread in head and neck cancer}

Pouymayou $\mathrm{B}^{1}$, Riesterer $\mathrm{O}^{1}$, Guckenberger $\mathrm{M}^{1}$, Unkelbach $\mathrm{J}^{1}$

${ }^{1}$ Radiation Oncology, University Hospital Zurich, Zurich, Switzerland

Aims: In head and neck radiotherapy, a large portion of the CTV aims at a prophylactic irradiation of the lymph node levels (LNL) at risk of harboring microscopic tumor despite the absence of visible metastases on imaging. We present a statistical model to personalize the probability of microscopic involvement of LNL based on the individual patient's state of tumor progression, i.e. we investigate how the risk of microscopic involvement depends on the location of macroscopic metastases.

Methods: We apply Bayesian Networks (BN) to model tumor progression. Each LNL is associated with 2 binary random variables, 1 for the hidden microscopic state of involvement and 1 for the observable macroscopic involvement. The probability of microscopic involvement is obtained by an inference algorithm. In this work we investigate ip- silateral lymphatic spread (levels Ib to IV) for oropharyngeal (T1-T2) tumors and determine the $\mathrm{BN}$ parameters via maximum likelihood estimation based on the progression patterns of 103 patients published by Sanguineti (IJROBP, 74(5), p1356-64, 2009).

Results: Assuming that the imaging technique has a sensitivity of 0.66 and a specificity of 0.87 (e.g. PET in a meta-analysis, Liao, BMC Cancer, 12:236, 2012), our model estimates the risk of microscopic involvement for level IV as 3.3\% for N0 patients, $4.1 \%$ if only level II is macroscopically involved, $8.4 \%$ if only level III is macroscopically involved, and $10.3 \%$ if both level II and III harbor macroscopic metastases. The corresponding risks of involvement for level III are $8.5 \%$, $16.0 \%, 54.6 \%$, and $71.3 \%$, respectively.

Discussion/Conclusion: The BN provides a statistical model to merge population-based data on lymphatic spread with the individual patient's state of progression. This personalized risk assessment could further support the CTV definition. This application to early-stage oropharyngeal cancer suggests that the risk of microscopic involvement of level IV is exceeding 5\% only when level III harbors metastases. Thus, level IV could be excluded from the elective CTV for N0-patients. Posterwalk

\section{Dosimetric commissioning of the proton beam model for PSI Gantry 3}

Böhlen TT ${ }^{1}$, Belosi $\mathrm{F}^{1}$, Rosas $\mathrm{S}^{1}$, Fredh $\mathrm{A}^{1}$, Fachouri $\mathrm{N}^{1}$, Bizzocchi $\mathrm{N}^{1}$, Zepter $\mathrm{S}^{1}$, Lucconi $\mathrm{G}^{1,2}$, Weber $\mathrm{DC}^{1}$, Lomax $\mathrm{T}^{1}$, Hrbacek $\mathrm{J}^{1}$

Centre for proton therapy, Paul Scherrer Institut, UniversitätsSpital Zürich, Zurich, Switzerland

Purpose: PSI Gantry 3 is entering clinical operation this year. It consists of the ProBeam Gantry (Varian) connected to the PSI beamline. This work presents the creation and dosimetric commissioning of the clinical pencil beam scanning beam model using the TPS Eclipse (Varian).

Materials and Methods: The Proton Convolution Superposition 13.7 beam model was created for an energy range of 70-229 MeV with 2 optional range shifters (RS) using dosimetric base data including: 19 depth-dose curves, 19 absolute dose measurements using the dose-area product formalism and 530 spot profiles in air (with and w/o RS). The established beam model was validated against an extended set of benchmarking data with increasing complexity. Apart from the base data, the validation data included geometrical and clinical fields in homogenous phantoms using various detectors and setups: reference dosimetry with (1) a Farmer IC (central measurement) and (2) a Markus IC (depth-dose), (3) CCD camera (high-resolution transversal measurement), (4) patient-specific QA (PSQA) setup (water column with PTW 2D array), as well as (5) complex cases (slab geometry, oblique incidence and anthropomorphic phantoms) using the PSQA setup and finally (6) end-to-end test with an anthropomorphic head phantom using films. Measurement results were compared against commissioning tolerances for beam model acceptance.

Results: Base data showed a good agreement with model predictions: ranges (R80\%) within $\pm 0.5 \mathrm{~mm}, \sigma_{\text {lat }}$ within $\pm 0.9 \mathrm{~mm}$. Farmer IC measurements (1) agreed within $\pm 3 \%$. For Markus IC measurements (2), $3 \mathrm{D}$ gamma pass rates $(3 \mathrm{~mm}, 3 \%)(\mathrm{G}(3,3))$ were $>90 \%$. CCD measurements (3) had a G(3,3) of $>90 \%$ and PSQA measurements (4) for geometrical and clinical field resulted in $\mathrm{G}(3,3)>90 \%$. Additional analysis and evaluations were performed.

Conclusion: All dosimetric commissioning tolerances were fulfilled and the beam model was accepted for clinical use. Poster 
Development and validation of Rapid-Plan model for prostate patient treatment

C. Negreanu ${ }^{1}$, A. Clivio ${ }^{2}$, L. Oliver ${ }^{1}$, E. Barletta ${ }^{1}$, H. Härle ${ }^{1}$, O. Sommer ${ }^{1}$, B. Schnekenburger ${ }^{1,2}$, R. Gräter ${ }^{2}$ and U. Meier ${ }^{1,2}$

${ }^{1}$ Klinik für Radio-Onkologie, Kantonsspital Winterthur, Winterthur, Switzerland

${ }^{2}$ Zentrum für Radiotherapie Rüti, Rüti ZH, Switzerland

Aim: The present work aims to generate and validate a Rapid-Plan (RP) dose-volume histogram (DVH) estimation model for prostate treatment planning. We would like to prove first that such a RP model-based plan can improve the quality of the clinical plans, and then to evaluate whether there could be an effective clinical benefit in planning with RP instead of the standard clinical planning.

Methods: A DVH estimation model has been configured based on plan and patient data extraction from a department database and the subsequent model training. Over thirty plans, optimized using the VMAT technique, with two arcs and various collimator angles were chosen, as they were considered optimal in terms of quality and the sparing of critical structures. An iterative choice of the optimization objectives as DVH lines or points, and their priorities was performed until the plan quality converged with that obtained by experienced planners. To validate the model 10 new patients were planed using the same VMAT technique both by experienced dosimetrist and based on the RP model, and then compared in terms of target coverage, specific sparing critical structures, and other technical aspects such as plan modulation.

Results: During model optimization it was found that the PTV coverage was minimally reduced in the superior and inferior directions for RP compared with clinical plans. After manual tuning of the parameters, the RP-model validation on similar cases showed a comparable target dose distribution, as well as slightly improved organ sparing with respect to the manually optimized plans.

Conclusion: We have shown that the RP model enabled the generation of clinically acceptable treatment plans without further planner intervention for 9 of the 10 prostate treatment cases considered. Moreover, planning time was in all cases reduced and no general increase in plan modulation has been found. Poster

\section{Statistical assessment of inspiration thresholds for DIBH left- breast irradiation}

Lia Vugts

Radio-Onkologie, Radio-Onkologie Zentrum KSA-KSB, Aarau, Switzerland

Introduction: At our centre, Deep inspiration Breath hold (DIBH) with Real-time Positioning Management (RPM) (Varian Motion Management System TB2.5) has been recently implemented. The used thresholds for DIBH have a direct influence on the treatment quality as well as on the treatment time. In the current study the breath hold curves of left breast cancer patients treated with the DIBH technique over six months are statistically analyzed to optimize the inspiration level and thresholds in order to get the best treatment quality by minimal interruptions and to assess the necessity and feasibility of either individualized or model based thresholds.

Methods: Individual breathing curves were recorded by the RPM system with the marker block placed on the chest wall of the contralateral breast. The measured amplitude is the motion of the marker block relative to a baseline set at exhale. The mean inspiration level between $15 \%$ and $85 \%$ "beam-on" time is analyzed per field for the individual patients. To optimize the thresholds, the deviation of the actual and the mean inspiration level at $15 \%, 50 \%$ and $85 \%$ of the "beam on" time is determined for the individual as well as the cohort of patients.

Results: Preliminary results indicate that the inspiration thresholds are stable $( \pm 2 \mathrm{~mm}$ ) between $15 \%$ and $85 \%$ of the "beam on" time over the whole treatment course for the cohort of patients. However the interfractional reproducibility of the level of inspiration is moderate. In the majority of patients it varies by about $2-3 \mathrm{~mm}$ in height and becomes more stable during the later fractions of the treatment course.

Conclusion: These observations let us project that better consistency in the inspiration level will allow smaller model based thresholds of about 1-2 mm without increasing the treatment time. For gain in inspiration level consistency criteria such as the patient setup, clinical guidelines and visual coaching will be considered. Poster

\section{CFR-PEEK compared to titanium implants for spinal stabilization may produce less dosimetric corruption during pencil beam scanning proton therapy delivery}

R. Poel ${ }^{1}$, F. Belosi ${ }^{1}$, A. Gisep ${ }^{2}$, F. Albertini ${ }^{1}$, M.Walser ${ }^{1}$, D. C. Weber ${ }^{1}$

${ }^{1}$ Center for Proton Therapy, Paul Scherrer Institute, Villigen,

Switzerland

${ }^{2}$ Icotec AG, Altstätten, Switzerland

Aims: Titanium implants are problematic in proton therapy (PT). Scatter and absorbance of particles, as well as CT artefacts, cause uncertainties in target definition, dose calculation and dose delivery. Despite corrections in planning, uncertainties remain.

Implants made from carbon fiber reinforced (CFR) polyetheretherketone (PEEK) are radiolucent and therefor do not produce artefacts on CT. Also, scatter and absorbance is highly reduced.

This study aims to assess the advantages of CFR PEEK vs. titanium implants in PT.

Methods: A unique anthropomorphic torso phantom was developed with an extractable spinal part. Two additional spinal inserts were developed containing a titanium and CFR PEEK spinal implant, consisting of a vertebral body replacement and two stabilizing rods with four pedicle screws each.

The three phantom cases received standard clinical workup of simulation, artefact correction, intensity modulated PT planning and delivery of a $2 \mathrm{~Gy}$ fraction to a defined target. The plans were optimized until they reached the same objectives resulting in comparable dose delivery.

The delivered dose was measured by Gafchromic film placed in a coronal plane through the spinal cord. Film analysis was performed on 4 ROIs in the target at the level of the pedicle screws.

Results: The film irradiated with the titanium implant showed cold spots correlating to the hardware. The dose in the ROIs locally dropped to $84.3 \%$ (SD 1.4\%) of the reference dose. The region receiving less than $95 \%$ of the reference dose covered $28.0 \%$ (SD 6.61\%) of the ROI. The relative minimum dose in the CFR PEEK case was $90.4 \%$ (SD $0.7 \%$ ), identical to the reference case. Only $6.6 \%$ (SD 1.9\%) of the ROIs was below $95 \%$ of the reference dose.

Conclusion: CFR PEEK produces a more homogeneous dose in IMPT delivery when compared to titanium. Despite corrections and good coverage on calculated plans, measured films in the titanium case showed substantial cold spots compromising the coverage, as opposed to CFR PEEK. Poster

\section{Commissioning of Varian GammaMed HDR ring applicators: Our experience with an innovative workflow}

E. Barletta ${ }^{1,2}$, H. Haerle$^{1}$, A. Clivio ${ }^{2}$, C. Negreanu ${ }^{1}$, O. Sommer ${ }^{1}$, B. Schnekenburger ${ }^{1}$, U. Meier ${ }^{1,2}$

${ }^{1}$ Department of Radiation Oncology, Kantonsspital Winterthur, Winterthur, Switzerland

${ }^{2}$ Zentrum für Radiotherapie Rüti Zürich-Ost-Linth AG, Rüti ZH, Switzerland

Aims: Aim of this work is to develop an alternative workflow for the HDR ring applicators commissioning, using materials and devices 
commonly available in a Radio-Oncology department. Varian has supplied a way for that, but, unfortunately, it involves the use of radiological films, nowadays not handy to use. Each ring applicator has to be commissioned in order to define the shift to apply in order to correct the systematic positioning deviation of the source introduced by the ring internal geometry.

Methods: Three different ring applicators were scanned in a conventional CT scanner using metal markers as geometrical references for both the applicator itself and its lumen. The images were imported in the Varian Brachytherapy workspace, where several test treatments plans were prepared, also relating the source position with the external markers. The plans were then treated by a Varian GammaMedPlus afterloader, using a Gafchromic film firmly fixed to the ring applicators. The position of the markers was also marked on the film surface. Finally, using them as references and the images of the source dwell on the film was possible to determine the shifts of the sources from the expected positions. The final results were calculated as statistical average of several measurements.

Results: The correction shifts for the different applicators were measured and found to be about $5 \mathrm{~mm}$ for the two $94.3 \mathrm{~mm}$ circumference applicators and $1.8 \mathrm{~mm}$ for the $81.7 \mathrm{~mm}$ ones. These values reflect the different length and the different curvature of the lumen.

Conclusion: The developed method was successfully applied for specific commissioning of the tested ring applicators. The removal of need to use radiological films will allow every Radio-Oncology department to commission these ring applicators and perform QA Programs easily and without additional costs. Poster

\section{Early evaluation of prostate positioning and movement during external beam radiation therapy using a transperineal 4D ultrasound system}

Marc Pachoud, Oscar Matzinger, Zohra Mazouni, Adrien Cosinschi, Sarah Godin and Sarah Ghandour

Radiotherapy, Service Interdisciplinaire de Cancérologie, Hôpital Riviera-Chablais, Vevey, Switzerland

Purpose: The Elekta Clarity ${ }^{\mathrm{TM}}$ transperineal 4D ultrasound system (4DUS) allows for real time imaging of the prostate during External Beam Radiation Therapy (EBRT). We evaluated the interfractional setup errors and intrafractional changes in prostate movement over an EBRT course.

Methods: Ten localized prostate cancer patients received image-guided EBRT delivered in 39 fractions. For each fraction, the prostate localization errors were controlled by 4DUS and CBCT. In total, interfractional setup errors of 390 ultrasound scans were assessed and compared to daily CBCT scans. Intrafraction motion evaluation was based on the continuous tracking of 69,996 prostate position readings along the left-right (LR), anterior-posterior (AP) and superior- inferior (SI) directions. The overall mean values and standard deviation (SD) along with systematic and random errors were calculated.

Results: The average discrepancies \pm SD of prostate positioning between 4DUS and CBCT were $-0.8 \pm 1.8 \mathrm{~mm}$ laterally, $0.1 \pm 3 \mathrm{~mm}$ vertically $0.6 \pm 1.8 \mathrm{~mm}$ longitudinally. The systematic error $(\Sigma)$ and random error $(\sigma)$ of 4DUS were $\Sigma=(1.3,1.1,0.7) \mathrm{mm}$ and $\sigma=(2.8,2.5,2.5)$ $\mathrm{mm}$ in LR, AP and SI directions, respectively. The mean $(\mu)$, the $\Sigma$ and the $\sigma$ of intrafraction prostate movement were $\mu=(0.02,-1.11,0.68)$ $\mathrm{mm}, \Sigma=(0.12,0.46,0.42) \mathrm{mm}$ and $\sigma=(0.57,0.67,0.58) \mathrm{mm}$. The percentage of treatments time for which the prostate movement was less than $2 \mathrm{~mm}$ was $98.5 \%, 87 \%$ and $96 \%$ in the LR, AP and SI directions, respectively.

Conclusion: 4DUS is comparable to CBCT based image guidance for prostate positioning with the advantage of avoiding extra radiation to the patient. Most of the prostate displacements were less than $2 \mathrm{~mm}$ which could be potentially important for dose escalation studies. Oral presentation
Motion-including dose reconstruction on 4DCT or patientspecific motion models validated on a time-resolved ground truth

J Bertholet ${ }^{1}$ B Eiben ${ }^{2}$ MJ Menten ${ }^{1}$ EH Tran ${ }^{2}$ DJ Hawkes ${ }^{2}$ S Nill ${ }^{1}$ JR McClelland ${ }^{2}$ U Oelfke ${ }^{1}$

${ }^{1}$ Joint Department of Physics, The Institute of Cancer Research and The Royal Marsden NHS Foundation Trust, London, Great Britain ${ }^{2}$ Centre for Medical Image Computing, Department of Medical Physics and Biomedical Engineering, University College London, London, Great Britain

Aims: Motion-including dose reconstruction (MIDR) can assess the delivered dose during radiotherapy using machine log-files and the time-resolved anatomy. We quantified MIDR accuracy using either a 4DCT or a patient-specific motion model (PSMM) as time-resolved anatomy by comparing both against a ground-truth.

Methods: A digital anthropomorphic phantom (XCAT) with a lung tumour was created and animated using a lung tumour trace measured in-vivo. A 10-phase 4DCT was simulated and used to generate a stepand-shoot treatment plan $(64 \mathrm{~Gy} / 32 \mathrm{Fx} / 6 \mathrm{MV})$ on the end-exhale phase. A PSMM was fitted to the unsorted axial 4DCT slices, relating the motion to the chest and diaphragm surrogate signals.

A later part of the motion trace was used to simulate treatment delivery on the moving anatomy with and without dynamic-MLC tracking. The intra-treatment time-resolved PSMM anatomy was estimated using the surrogate signals. The treatment fluence was discretized into sub-beams. Each sub-beam was associated to the XCAT/PSMM instance or 4DCT phase at which it was delivered. Sub-beam isocenters were shifted to account for residual tumour position differences. The dose of each instance/phase was calculated in a commercial TPS using a Monte Carlo dose engine and accumulated on the reference instance/ phase. PSMM- and 4DCT-based MIDR were compared to the XCATground-truth.

Results: For XCAT, PSMM and 4DCT respectively: PTV $\mathrm{D}_{95 \%}$ was $62.05 / 62.99 / 62.21$ Gy, lung $\mathrm{V}_{20 \mathrm{~Gy}}$ was $22.05 / 22.49 / 21.92 \%$, heart $\mathrm{V}_{40 \mathrm{~Gy}}$ was $5.94 / 5.92 / 5.80 \%$ with tracking and PTV $\mathrm{D}_{95 \%}$ was $51.92 / 52.99 / 53.14$ Gy, lung $\mathrm{V}_{20 \text { Gy }}$ was $22.02 / 22.36 / 21.65 \%$, heart $\mathrm{V}_{40 \mathrm{~Gy}}$ was 4.73/4.70/4.70\% without tracking.

Conclusions: PSMM- and 4DCT-based MIDR had similar accuracy for typical breathing. Target dose reconstruction was less accurate without than with tracking. MIDR accuracy for less regular breathing patterns can be evaluated with the presented method. Posterwalk

\section{Plan comparison of iris and incise $\mathbf{2}$ multi leaf collimator-based} stereotactic radiotherapy for brain metastasis

Ermiş $\mathrm{E}^{1}$, Leiser $\mathrm{D}^{1}$, Cihoric $\mathrm{N}^{1}$, Schmidhalter $\mathrm{D}^{2}$, Henzen $\mathrm{D}^{2}$, Malthaner $\mathrm{M}^{2}$, Blatti-Moreno $\mathrm{M}^{1}$, Tsikkinis $\mathrm{A}^{1}$, Aebersold $\mathrm{DM}^{1}$, Herrmann $\mathrm{E}^{1}$

${ }^{1}$ Department of Radiation Oncology, Inselspital, Bern University Hospital, and University of Bern, Bern, Switzerland

${ }^{2}$ Division of Medical Radiation Physics, and Department of Radiation Oncology, Inselspital, Bern University Hospital, and University of Bern, Bern, Switzerland

Aims: Second generation multi leaf collimator (MLC) has been introduced for the CyberKnife Robotic Radiosurgery System (Accuray Inc., Sunnyvale, USA) in 2016. The aim of the present study was to compare the plan quality between InCise 2 MLC and IRIS by analyzing dosimetric parameters for small and large brain targets.

Methods: Ten brain metastasis targets were selected. Treatment plans were generated with IRIS and InCise 2 MLC (Multiplan 5.3 or Precision 1.1). The following parameters were analyzed: coverage, minimum, mean and maximum dose of PTV and OAR, conformity index (CI), Nakamura CI (nCI), homogeneity index (HI), gradient index 
(GI), V12 Gy, treatment time, number of beams, nodes and monitor units (MU). Values were normalized to the prescribed dose. Paired T-test was used to compare the parameters.

Results: The mean volume of the lesions were $19.5 \mathrm{~cm}^{3}$ (range $\left.2.3-56.8 \mathrm{~cm}^{3}\right)$. CI and nCI were significantly lower with IRIS plans ( $p=0.048$ and $p=0.033$ ), whereas GI values were higher in MLC plans $(p<0.01)$. No significant patterns of conformity and gradient differences upon target shape (circular or irregular) and tumor volume (threshold of $10 \mathrm{~cm}^{3}$ ) were observed. PTV mean doses were higher with IRIS plans $(p=0.03)$. The number of MUs, beams and nodes were significantly lower with MLC plans resulting decreased treatment time $(p<0.01)$. No differences were observed in terms of coverage, HI, OAR dose distributions and V12. More detailed results will be presented during the meeting.

Conclusion: Results of the study showed that Incise 2 MLC plans for brain metastases were dosimetrically feasible. MLC plans have steeper dose falloff, which could be an advantage for an OAR near the target. Treatment time of the MLC plans were 30\% lower, which increase the delivery efficiency. Poster

\section{Improvements in cranio-spinal-axis (CSA) treatments at PSI Gantry2 (G2)}

Mikroutsikos L, Gonzalo R, Belosi F, Lomax T, Weber DC, Bolsi A

Center for Proton Therapy, Paul Scherrer Institut, Villigen, Switzerland

Purpose: CSA irradiations have been performed at PSI on the first Pencil Beam Scanning (PBS) Gantry1 (G1), since 2004. Due to its technical limitations, only pediatric patients in prone position could be treated. The new gantry (G2) in clinical operation since 2014 is not affected by these limitations, therefore adult patients and in supine position could be irradiated, thus increasing the number of patients potentially treated with CSA and increasing patient comfort. The purpose of this work was to investigate the planning technique for $\mathrm{G} 2$, considering supine positioning, smaller spot dimensions and different spot spacing as compared to G1.

Methods: All 17 patients treated in G1 since 2004 were re-planned on the G2 TPS (PSIplan, in-house developed). For each case several optimization parameters (i.e. margin for spot positioning outside PTV) and different planning techniques were tested: Single Field Uniform Dose (SFUD), IMPT (Intensity Modulated Proton Therapy), preabsorber fixed in the beam line or inserted only for superficial spots and pre-absorber close to the patient (virtual pre-absorber). G1 and G2 plans were compared in terms of targets' V95\%, Homogeneity Index (HI) and OARs sparing.

Results: Improvement in target coverage for the G2 plans was found for IMPT plans with $4 \mathrm{~mm}$ margins outside PTV for spot positioning and virtual preabsorber (G2 PTV mean V95\% is $99.1 \pm 0.3 \%$ vs $88.6 \pm 1.5 \%$ for G1). G2 plans resulted in more homogeneous dose distributions (target volume average is $10.4 \pm 0.5 \%$ ), while $25.9 \pm 8.8 \%$ for G1. G2 plans presented lower or comparable D2\% for intra cranial OARs as compared to $\mathrm{G} 1$ results; $\mathrm{G} 2$ plans provided significantly better sparing of extra-cranial OARs due to the sharper lateral and distal fall-off of the G2 proton beam.

Conclusion: G2 dose distributions demonstrated not only the feasibility of CSA treatments in the newest system but also an improved dosimetric plan quality in respect to G1. Since October 2017, 6 patients have been treated in G2. Poster
Contraindication for radiative deep regional hyperthermia for patients with large carbon implants

Marder D. ${ }^{1}$, Poel R. ${ }^{2}$, Gisep A. ${ }^{3}$, Van Stam G. ${ }^{1}$, Timm O. ${ }^{1}$, Puric E. ${ }^{1}$, Datta N.R. ${ }^{1}$, Lutters G. ${ }^{1}$

${ }^{1}$ Radio-Onkologie-Zentrum KSA-KSB, Kantonsspital Aarau, Aarau, Switzerland

${ }^{2}$ Paul Scherrer Institute, Villigen, Switzerland

${ }^{3}$ icotec AG, Altstätten, Switzerland

Aims: Hyperthermia to temperatures of $41-43{ }^{\circ} \mathrm{C}$ has shown to be a valuable sensitizer for radiation- and chemo-therapy in cancer treatment. In this study the influence on specific absorption rate (SAR) and temperature by carbon fiber reinforced polyether-ether-ketone (CFRPEEK) implants was investigated.

Methods: The influence on SAR and temperature was investigated in three rods with identical diameter of $5.5 \mathrm{~mm}$ and length of $140 \mathrm{~mm}$. The rods were made of a titanium (TI), CFR-PEEK and PEEK of which the TI and the CFR-PEEK are commercially available products for trauma and reconstructive surgery provided by icotec AG, Altstätten, Switzerland. For the SAR measurements the samples were placed in a phantom inserted in the SigmaEye applicator of the BSD2000 3D deep hyperthermia equipment (Pyrexar Medical, Salt Lake City, USA). Inside the phantom, a SAR probe (EASY4/MRI, SPEAG, Zürich, Switzerland) was moved along the samples and the relative SAR strength was recorded. For the temperature measurements, the samples were placed in a tissue equivalent gel. The temperature rise following a power pulse of $1000 \mathrm{~W}$ for $5 \mathrm{~min}$ (pulse\#1) was measured. Results: The SAR measurements parallel to the axis of the rods showed a clear increase of maximum relative SAR strength for both TI and CFR-PEEK but no increase for the PEEK material. Relative SAR increased by $329 \%$ for TI and $297 \%$ for CFR-PEEK at the tips of the rod. Temperature measurements in the gel phantom showed a similar behavior with a maximum temperature rise at the tips of the rod up to $27{ }^{\circ} \mathrm{C}$ for both TI and CFR-PEEK and $3{ }^{\circ} \mathrm{C}$ for PEEK after pulse\#1.

Conclusion: TI and CFR-PEEK show a similar influence on SAR and temperature in a deep hyperthermia treatment set-up. Thus CFR-PEEK implants, like metal implants, must be a contraindication for radiative deep hyperthermia treatment. Poster

\section{Dosimetric study of automatic brain metastases planning: Comparison of Varian HyperArc with BrainLab Elements}

Leick M., Lomax N., Alonso S., Hancock T., Rogers S., Lutters G.

Radio-Onkologie-Zentrum KSA-KSB, Kantonsspital Aarau, Aarau Switzerland

Aim: To investigate plan quality indices for two different techniques available for Automatic Brain Metastases Planning (ABMP): BrainLAB Elements (BEL) with dynamic conformal arcs (DCA) and Varian HyperArc (VHA) with volumetric modulated arcs (VMAT). Both treatment planning systems allow the automatic planning workflow for multiple targets using one single isocentre with multiple coplanar and non-coplanar arcs.

Methods: New plans were generated with Eclipse v15.5 for 10 clinical cases of multiple small brain metastases (with 2 to 4 targets), which had been originally planned with Elements version 1.2 using dynamic conformal arcs (DCA).

HyperArc uses a VMAT optimised technique with up to 5 arcs. Each plan was optimised to match the same prescription dose and D99\% for each target as in BEL. Treatment plan dose distributions were evaluated using "Paddick" conformity index (PCI) and Gradient Index (GI). Results: All new HyperArc plans met our standards for clinical acceptability, including minimum target coverage and maximum dose limits to critical organs at risk. In the original BEL plans using DCA, the quality indices were $\mathrm{PCI}=0.61+/-0.11$ and $\mathrm{GI}=3.46+-1.2$. There 
was higher conformity in the simulated HyperArc VMAT plans, with the mean $\mathrm{PCI}=0.79+/-0.14$, but lower dose gradients, with the mean $\mathrm{GI}=3.57+/-1.2$.

Conclusion: Using automated planning features, both treatment planning systems consistently achieved conformal plans with high dose gradients for multiple targets. HyperArc (VMAT) plans had better conformity indices and also offer the possibility to reduce the treatment time with delivery process automation. BrainLab Elements (DCA) plans achieved better gradient indexes for small target volumes. Poster

\section{Assessing accuracy and efficiency of Monte Carlo based dose calculation for MLC CyberKnife treatments}

P.-H. Mackeprang ${ }^{1}$, W. Volken ${ }^{1}$, D. Henzen ${ }^{1}$, D. Schmidhalter ${ }^{1}$, M. Malthaner ${ }^{1}$, S. Mueller ${ }^{1}$, D. Frei ${ }^{1}$, W. Kilby ${ }^{2}$, D. M. Aebersold ${ }^{1}$, M. K. Fix ${ }^{1}$, P. Manser ${ }^{1}$

${ }^{1}$ Division of Medical Radiation Physics and Department of Radiation Oncology, Inselspital, Berne University Hospital, Bern, Switzerland ${ }^{2}$ Accuray Incorporated, Sunnyvale, California, USA

Aims: To assess the accuracy and efficiency of the recently commercially launched MC based dose calculation engine for MLC based treatments in the CyberKnife treatment planning system (PrecisionMC).

Methods: Dose distributions in water of 11 different MLC shaped fields and of treatment plans for 3 phantom and 7 clinical lung cases are calculated with PrecisionMC and an in-house vendor-independent EGSnrc MC based dose calculation framework (IDC) used as a benchmark for comparison. Dose distributions in water are also compared to measurements. The lung PTVs ranged from $14 \mathrm{~cm}^{3}$ to $93 \mathrm{~cm}^{3}$. For dose distributions of treatment plans, 3D gamma analysis using $2 \% / 1 \mathrm{~mm}$ criteria with a global $10 \%$ dose threshold as well as dose-volume parameters are used for analysis. Efficiency is assessed by time to calculate dose distributions to a mean statistical uncertainty of 1.7-2.2\% (targeted uncertainty of $2 \%$ ) for voxels receiving $>50 \%$ of Dmax. As IDC is not optimized for efficiency but used as a gold standard for comparison, calculation time is not assessed but given for reference.

Results: Dose profiles in $15 \mathrm{~mm}$ depth as well as depth dose curves in water show agreement between PrecisionMC and IDC within 3\% or $1 \mathrm{~mm}$. For the phantom and clinical cases, dose distributions calculated by PrecisionMC and IDC agree with gamma passing rates of $>=97.2 \%$, mean PTV doses agree within $-1 \%$ to $2.3 \%$. For clinical lung cases, lung V20 agrees within $+/-1.5 \%$. PrecisionMC calculation times were $<2.7 \mathrm{~min}$ for clinical lung cases and medium resolution calculation (calculation voxel size doubled in CT x- and y-direction), equaling calculation time below $9.5 \mathrm{~min}$ in native $\mathrm{CT}$ resolution on a 12 core machine. IDC calculations took $4-6 \mathrm{~h}$ on a 100 core computing cluster.

Conclusion: Both accuracy and efficiency of PrecisionMC were benchmarked against MC based IDC and are within clinically accepted limits. Poster

\section{Breast with axilla treatment: Comparison between 5 fields and VMAT technique}

A.Clivio $^{2}$, C.Negreanu ${ }^{1}$, E.Barletta ${ }^{1,2}$, H.Haerle ${ }^{1}$, O.Sommer ${ }^{1}$, B.Schnekenburger ${ }^{1}$, U.Meier ${ }^{1,2}$, R.Graeter ${ }^{2}$

${ }^{1}$ Klinik für Radio-Onkologie, Kantonsspital Winterthur, Winterthur, Switzerland

${ }^{2}$ Zentrum für Radiotherapie Rüti, Rüti ZH, Switzerland

Aim: We aim to demonstrate the possibility to using the VMAT instead of the isocentrical 5 fields IMRT (5F) for the irradiation of breast patients after breast conserving therapy (BCS) or mastectomy including the axillary lymphnod region. We want to explore, whether with VMAT plans we can spare better the organs, especially the contralat- eral breast, the heart and the lung, avoiding the junction and reducing the treatment time.

Methods: We planned with VMAT 20 patients treated previously with the 5F, 9 of them after mastectomy, 9 for right breast and 11 for left breast. The prescribed dose is 50 Gy in 25 fractions. The VMAT plans (2 partial arcs with $6 X$, with a collimator about $\pm 10-15$ ) are calculated by enlarging $1 \mathrm{~cm}$ the planning $\mathrm{CT}[1]$. For the $5 \mathrm{~F}$ technique we used one isocenter in the cranial region, 2 tangential IMRT fields with the upper jaw close to the isocenter with $6 \mathrm{MV}$, that cover the thorax, and 3 static fields $\left(0^{\circ}, 180^{\circ}\right.$ and one medial field) with 6,10 or $15 \mathrm{MV}$ to cover the axilla.

Results: For the PTV coverage, the 5F plans show a slightly superior dose coverage, with a $\mathrm{V}_{95 \%}[\%]$ of $95.9 \pm 1.1$ and $95.6 \pm 1.2$ for VMAT. The same result is valid for $\mathrm{D}_{98 \%}$. For the ipsilateral lung dose the $\mathrm{V}_{5}[\%]$ and $\mathrm{V}_{20}[\%]$ are monitored and we observed for $5 \mathrm{~F} 34.0 \pm 6.8$ and $12.2 \pm 4.0$ and $45.2 \pm 6.1$ and $9.8 \pm 2.6$, for VMAT respectively. For the contralateral lung and breast we obtained for the mean dose [Gy] $0.1 \pm 0.1$ and $1.2 \pm 0.3,0.1 \pm 0.1$ and $1.2 \pm 1.4,(5 \mathrm{~F}$ and VMAT) and for the heart for the left side patients $0.4 \pm 0.2$ and $1.5 \pm 0.4$ and for right side $1.4 \pm 0.5$ and $2.7 \pm 0.7$ ( $5 \mathrm{~F}$ and VMAT).

In addition with VMAT technique we can reduce the MU by about $29 \%$ and the treatment time by about $53 \%$.

Conclusion: With VMAT technique we have less uncertainties for the field junctions, with less irradiation time and comparable dose coverage for the PTV and contralateral organs sparing.

\section{References}

1. Nicolini et al (2011) Medical Physics 38:4025

\section{Comparison of manual and two automated planning solutions for stereotactic brain radiosurgery}

M. Zamburlini' ${ }^{1}$, J. Krayenbuehl ${ }^{1}$, A. Sonbadhar ${ }^{1}$, D. V. McDonnell ${ }^{1}$, M. Guckenberger ${ }^{1}$, N. Andratschke ${ }^{1}$

${ }^{1}$ Department of Radiation Oncology, University Hospital Zurich, Zurich, Switzerland

Aims: Stereotactic radiosurgery (SRS) treatment planning is challenging, because adequate beam geometry is patient and lesion dependent, which is particularly complex in the treatment of multiple BM (mBM) with one single isocenter. In this in-silico planning study, we compared two dedicated automatic treatment planning solutions (TPS) for BM with manual optimization.

Methods: The planning study is based on 10 randomly selected patients treated with SRS at our hospital. For comparison purposes, the patients were classified in 3 groups: single $\mathrm{BM}(n=3)$; $3-5 \mathrm{BM}(n=4)$ and $>10 \mathrm{BM}(n=3)$. The study compared manually generated treatment plans (CP) (Eclipse v13.6) with Hyperarc (HA) (v 15.5 Varian Medical Systems, Palo Alto, USA) and two versions of Elements Multiple Brain Mets (EL1.5 and EL2.0) (v1.5 and alpha v2.0, Brainlab, Germany). All plans were prepared for a TrueBeam linac with HDMLC and a single isocenter. Plans were normalized to the PTV to have D100\% > 99\%. An inhomogeneous dose prescription was used with at least $113 \%$ of the prescribed dose (PD) covering $95 \%$ of each GTV and a Dmax of $125 \%$ of the PD. Plans were compared based on target mean dose, conformity index (CI) and dose to normal tissue.

Results: HA, EL2.0 and CP achieved similar target coverage. However, EL1.5 was not able to achieve the prescribed dose inhomogeneity. EL1.5 plans were therefore re-normalized to achieve comparable GTV coverage. EL1.5 showed less a favorable CI, due to the increased normalization to reach the necessary GTV mean dose. In the case of $>10$ BM, EL1.5 and EL2.0 showed better brain sparing. Effective planning time is less than 5 min for EL and HA provides a gain in effective planning time due to the automatic selection of beam geometry. 
Conclusions: EL and HA are able to provide clinically acceptable plans for SRS of single and $\mathrm{mBM}$, reducing planning time substantially compared to manual planning. Poster

\section{Commissioning of a commercial table on ProBeam Gantry3 using laser tracker}

\section{M.Peroni ${ }^{1}$, A. J.Lomax ${ }^{1}$, D. C.Weber ${ }^{1,2,3}$ and S. Safai ${ }^{1}$}

${ }^{1}$ Center for Proton Therapy, Paul Scherrer Institute, Villigen, Switzerland; ${ }^{2}$ Radiation Oncology Inselspital, Bern, Switzerland; ${ }^{3}$ Radiation Oncology, University Hospital of Zurich, Zurich, Switzerland

Aims: For accurate radiotherapy, a reliable table ensuring daily reproducible positioning is crucial. In particular, not only point-to-point moves, but also rotations around the isocentre need to be accurate, as non-coplanar fields are often used. This should be the case regardless of localisation and patient weight.

Methods: We present a method to characterize the table in the Varian ProBeam proton therapy system using Laser Tracker (LT), which guarantees an accuracy of measurement up to $30 \mu \mathrm{m}$. The table consists of a QFix k-Vue interface and couch top mounted on a KUKA robot (resolution $0.2 \mathrm{~mm}$ and $0.1^{\circ}$ ). 3 realistic human body weight distribution $(39.1 \mathrm{~kg}, 135 \mathrm{~kg}$ and $151.2 \mathrm{~kg}$ ) and 5 target points (head, shoulder, sternum, right and left hips) are measured. Target setup error is $<0.1 \mathrm{~mm}$. 3D distances from nominal isocentre for each weight and target in the presence of (i) isocentric rotations (every $30^{\circ}$ ) w. and wo. $1^{\circ}$ pitch and $1^{\circ}$ roll, as well as accuracy of (ii) pitch and roll and (iii) small $(<1 \mathrm{~cm})$ and large $(>1 \mathrm{~cm})$ point-to-point moves were also measured as well as repeatability and the influence of a $20 \mathrm{~cm}$ center of mass shift at $135 \mathrm{~kg}$ for (i). Conclusion: 3D residual distance is below $1 \mathrm{~mm}$ (range $0.03 \mathrm{~mm}$ $0.67 \mathrm{~mm}$ ) for all angles, weight classes and targets during isocentric rotations, even with pitch and roll. Repeatability of average deviations in (i) over 3 months, and shifting the centre of mass further from the last KUKA joint can introduce up to $0.5 \mathrm{~mm}$ deviation. Maximum deviation between nominal and measured pitch and roll is $0.03^{\circ}$ at $151.2 \mathrm{~kg}$. While the direction of rotation has no influence, the distance from the last joint of the KUKA robot can be challenging. During point-to-point moves, a shift $<1 \mathrm{~cm}$ could result in a deviation between set and measured distance of up to $50 \%$ at the target with the large arm. Overall, the accuracy of the table is satisfactory, as image guidance would not be able to detect deviations in patient position $<0.5 \mathrm{~mm}$. Poster

\section{Dosimetric impact of endorectal balloon on erectile function preservation in prostate SBRT}

M. Jaccard ${ }^{1}$, G. Lamanna ${ }^{1}$, A. Dubouloz ${ }^{1}$, M. Rouzaud ${ }^{1}$, R. Miralbell ${ }^{1,2,3}$ and T. Zilli ${ }^{1,2}$

${ }^{1}$ Radiation Oncology, University Hospital of Geneva, Geneva, Switzerland; ${ }^{2}$ Faculty of Medicine, Geneva University, Geneva, Switzerland; ${ }^{3}$ Radiation Oncology, Teknon Oncologic institute, Barcelona, Spain

Aims: Studies have shown that vessel-sparing radiotherapy for prostate cancer patients can preserve erectile function (EF). In this dosimetric study, we aimed to investigate the influence of endorectal balloons (ERB) on EF preservation by evaluating their role in dose sparing of the internal pudendal arteries (IPA) in planning the treatment for prostate SBRT.

Methods: Simulation CTs, both with and without ERB, were acquired for twelve patients with localized prostate cancer. PTV were planned to receive $5 \times 7.25$ Gy with two full VMAT arcs within well-defined OAR dose constraints (rectal and bladder wall and femoral heads). IPA were contoured on each CT using MRI registration. We used a knowledge-based software (RapidPlan ${ }^{\mathrm{TM}}$, Varian) to create 24 initial plans without any dose constraints on IPA. Further stepwise optimiza- tions were performed on each scanner to optimally reduce the IPA dose while respecting PTV homogeneity limit. IPA doses were compared between groups using non-parametric tests for paired samples.

Results: The analysis of the initial plans showed that median mean IPA dose was significantly lower with $(10.5 \mathrm{~Gy})$ than without $(12.8 \mathrm{~Gy})$ ERB $(p=0.023)$. The optimization process highly reduced the mean IPA dose $(-60 \%)$ without degrading PTV homogeneity and OAR dose sparing, both with and without ERB. A comparison of the best-optimized plans, with or without ERB, revealed that ERB obtained the best sparing of IPA (median mean dose of $4.1 \mathrm{~Gy} v s .5 .7$ Gy without, $p=0.006$ ).

Conclusion: ERB for prostate SBRT help to spare significantly the IPA compared to treatment plans generated without ERB. Since IPA appear to be critical structures for EF preservation, their dose sparing in the SBRT treatment planning process is crucial, even though dose constraints guidelines are yet unavailable. Posterwalk

\section{Potential benefits and feasibility of a surface-scanner for the production of 3D-printed bolus prior to planning CT: Tests on healthy volunteers}

Dipasquale G. ${ }^{1}$, Poirier A. ${ }^{2}$, Sprunger Y. ${ }^{2}$, Uiterwijk J. ${ }^{3}$, Miralbell R. ${ }^{1}$

${ }^{1}$ Department of Radiation Oncology, Geneva University Hospital, Geneva, Switzerland

${ }^{2}$ Medical School, Geneva University, Geneva, Switzerland

${ }^{3}$ IT consultant, Geneva, Geneva, Switzerland

Background: This study investigates the feasibility of surface-scanning to produce, prior to the planning computed tomography (CT) scan, a 3D-printed bolus. This should improve not only the workflow efficiency but also the treatment quality, with boluses being CT scanned at simulation allowing for quality control of their shape and density. Methods: Tests on healthy volunteers using a surface-scanner (Handy-

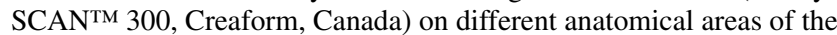
human body investigated: a) the influence of skin reflection on image acquisition; b) the ease of use; c) the acquisition time and; d) the bolus fitting. The post-processing software VXelements was used to create the bolus from the surface scans. The bolus models were 3D-printed in-house (Ultimaker 3 Extended, Ultimaker, Netherlands) using polylactic acid. To avoid the use of radiation on healthy volunteers, bolus fitting was evaluated by visually inspecting bolus-skin contact through purpose-made holes in the boluses and by questioning the volunteers on its comfort.

Results: Tests showed good and comfortable fit of boluses produced from surface-scanner images acquired in 0.6 to $7 \mathrm{~min}$. Image acquisition time depended on the clearance for the scanner to move around the body region and its shape. Surface-scanner boluses were also created for complex surface regions of the body such as ears and fingers and in deep inspiration breath hold conditions. None of the 3D-printed bolus required enlarging or shrinking of the initial model acquired in-vivo. Conclusions: Tests on volunteers suggest the possibility to produce high quality 3D-printed boluses with an excellent fit even for complex surface regions, suggesting the possibility to improve workflow efficiency, patient comfort and treatment quality. Poster

\section{Beyond the clonogenic cell survival assay: How the steady state of a process conceals dynamic properties of the DNA damage and repair process}

M. S. Weyland ${ }^{1}$, P. Thumser-Henner ${ }^{2,3}$, K. J. Nytko ${ }^{2,3}$, C. Rohrer Bley ${ }^{2,3}$, R. M. Füchslin ${ }^{1}$, S. Scheidegger ${ }^{1}$

${ }^{1}$ Institute of Applied Mathematics and Physics, ZHAW School of Engineering, Zurich University of Applied Sciences, Winterthur, Switzerland

${ }^{2}$ Division of Radiation Oncology, Vetsuisse Faculty University of Zurich, Zurich, Switzerland 
${ }^{3}$ Center for Applied Biotechnology and Molecular Medicine (CABMM), University of Zurich, Zurich, Switzerland

Aim: The aim of this work is to investigate the relationship between the damage after hyperthermia-radiotherapy (HT-RT) predicted by a dynamic DNA damage-repair model in silico as a function of time, and the final fraction of surviving cells.

Methods: Cell cultures and clonogenic cell survival assays were performed in vitro following standard protocols for Abrams osteosarcoma cells after optional hyperthermia treatment $\left(1 \mathrm{~h}, 42^{\circ} \mathrm{C}\right)$ and radiotherapy (0, 3, 6 Gy). Parameters for the Multi-Hit-Repair model [1] were found by fitting to this experimental data using differential evolution algorithms. Cell survival and time-course of DNA damage after HT-RT for varying rate parameters were compared.

Results and conclusion: (1) We show that the dynamics of DNA damage and repair are inaccessible by the study of survival curves, which merely represent the endpoint of the process. One example for such dynamics is that the model predicts a repair time of $1-3 \mathrm{~h}$, during which cells are eliminated and damage is cured. (2) However, this repair time can be varied extensively by multiplying the rate parameters by a constant. This is because after initial perturbations by hyperthermia and radiotherapy, a process at half the rate requires double the time to reach the same steady state. It is this final steady state which is captured by the clonogenic assay and thus, time-resolved experimental studies seem unavoidable to learn more about the dynamics of DNA damage and repair.

\section{References}

1. Scheidegger S et al. (2013), Using state variables to model the response of tumour cells to radiation and heat: A novel multi-hit-repair approach. CMMM, 16.

\section{Pre-treatment and in vivo measure of the leaf open times from the exit fluence in tomotherapy}

Mathieu Schopfer ${ }^{1}$, François Bochud ${ }^{1}$, Jean Bourhis ${ }^{2}$, and Raphaël Moeckli

${ }^{1}$ Institute of Radiation Physics, Lausanne University Hospital and University of Lausanne, Lausanne, Switzerland ${ }^{2}$ Radiation-oncology department, Lausanne University Hospital and University of Lausanne, Lausanne, Switzerland

Aims: To measure the leaf open time (LOTs) in tomotherapy from the exit fluence collected by the on-board detector (OBD) on a pulse-bypulse $(\mathrm{PbP})$ basis, in air and in vivo, for all delivery modalities. To investigate the inherent feasibility limits.

Materials and Methods: In tomotherapy, the beam modulation is performed by a binary MLC. The PbP data are collected per LINAC pulse (at $300 \mathrm{~Hz}$ ), i. e. each $3.3 \mathrm{~ms}$. The $\mathrm{PbP}$ data resolution is higher than the typical leaf "open-close" travel time (15 ms).

An in-house algorithm was developed to measure the LOTs from the $\mathrm{Pbp}$ data of the OBD. It normalises the $\mathrm{PbP}$ data to wipe off the patient attenuation and the beam modulation.

To test the algorithm, data were measured in air and in vivo for various tumour localisations. All procedures were planned in dynamic jaw mode. The data were collected on two tomotherapy units equipped with different detector models.

Results: The method accuracy is equal to the measurement window of the OBD, i.e. $3.3 \mathrm{~ms}$. The LOT discrepancies were measured between $-25 \mathrm{~ms}$ and $45 \mathrm{~ms}$, with means between -1.9 and $1.5 \mathrm{~ms}$ and with standard deviations between 2.4 and $5.4 \mathrm{~ms}$.

The largest differences correspond to leaves which do not open (or close) as expected when the planned LOT (or planned closing time between two openings) is short (below $45 \mathrm{~ms}$ ). The method worked as efficiently in air as in vivo.
Procedures with a $5.05 \mathrm{~cm}$ field are intrinsically problematic. The beam is shifted off the OBD at the beginning and at the end of the delivery. In such cases, the LOTs cannot be measured when the jaws aperture is narrower than $13 \mathrm{~mm}$, meaning that no LOT can be measured during the first and last 9 to $11 \mathrm{~mm}$ of the delivery.

Conclusion: This study fills a gap in the literature by demonstrating the full potential of measuring the LOTs from the exit fluence in tomotherapy, as the proposed method works under all planning and delivery conditions and proved to be accurate. It also confirmed existing results regarding the MLC behaviour.

Additionally, the method allows for setting up a MLC machine QA, a fast patient plan pre-treatment and in vivo QA, and allows for calculating the actual delivered dose per treatment fraction. Oral presentation

\section{Dosimetric suitability of dynamic mixed beam radiotherapy (DYMBER) for head and neck and brain treatments}

S. Mueller ${ }^{1}$, P. Manser ${ }^{1}$, W. Volken ${ }^{1}$, D. Frei ${ }^{1}$, E. Herrmann ${ }^{1}$, O. Elicin' ${ }^{1}$, D. M. Aebersold ${ }^{1}$, M.F. M. Stampanoni ${ }^{2}$, M. K. Fix ${ }^{1}$

${ }^{1}$ Division of Medical Radiation Physics and Department of Radiation Oncology, Inselspital, Bern University Hospital, and University of Bern, Bern, Switzerland

${ }^{2}$ Institute for Biomedical Engineering, ETH Zürich and PSI, Villigen, Switzerland

Aims: To investigate the dosimetric suitability of dynamic mixed beam radiotherapy (DYMBER) for head and neck and brain treatment plans. Methods: An in-house developed treatment planning process allows to create DYMBER plans consisting of photon dynamic trajectories (DTs) and step and shoot electron apertures collimated with the photon multileaf collimator (pMLC). Thus, no electron applicator is used. The gantry and table rotations of the photon DTs are determined such that the overlaps of the OARs with the target are minimized. The associated dynamic collimator rotation is established with minimized area between the pMLC leaves and the target contour. pMLC sequences of the photon DTs are first simultaneously optimized with the electron apertures and then re-optimized after final dose calculation of the electron apertures. DYMBER plans are created for three head and neck cases and one brain case. The deliverable Monte Carlo dose distributions of these plans are compared to those of dynamic trajectory radiotherapy (DTRT) plans consisting only of photon DTs and to VMAT plans. Results: For each case, the dose homogeneity in the target for the DYMBER plans is at least as good as for the DTRT and VMAT plans. Averaged over all four cases, the mean dose to the parallel OARs is $17 \%$ and $28 \%$ lower, D2\% to the serial OARs is $14 \%$ and $31 \%$ lower and V10\% to normal tissue is $13 \%$ and $11 \%$ lower for the DYMBER plans compared to the DTRT and VMAT plans, respectively.

Conclusion: The DYMBER plans dosimetrically outperformed the DTRT and VMAT plans for the investigated four cases indicating that head and neck and brain treatments could substantially benefit from DYMBER. This work was supported by Varian Medical Systems. Posterwalk

Plan comparison of iris and incise $\mathbf{2}$ multi leaf collimator-based stereotactic radiotherapy for brain metastasis

Ermiş $\mathrm{E}^{1}$, Leiser $\mathrm{D}^{1}$, Cihoric $\mathrm{N}^{1}$, Schmidhalter $\mathrm{D}^{2}$, Henzen $\mathrm{D}^{2}$, Malthaner $\mathrm{M}^{2}$, Blatti-Moreno $\mathrm{M}^{1}$, Tsikkinis $\mathrm{A}^{1}$, Aebersold $\mathrm{DM}^{1}$, Herrmann $\mathrm{E}^{1}$

${ }^{1}$ Department of Radiation Oncology, Inselspital, Bern University Hospital, and University of Bern, Bern, Switzerland

${ }^{2}$ Division of Medical Radiation Physics, and Department of Radiation Oncology, Inselspital, Bern University Hospital, and University of Bern, Bern, Switzerland 
Aims: Second generation multi leaf collimator (MLC) has been introduced for the CyberKnife Robotic Radiosurgery System (Accuray Inc., Sunnyvale, USA) in 2016. The aim of the present study was to compare the plan quality between InCise 2 MLC and IRIS by analyzing dosimetric parameters for small and large brain targets.

Methods: Ten brain metastases were selected. Treatment plans were generated with IRIS and InCise 2 MLC (Multiplan 5.3 or Precision 1.1). The following parameters were analyzed: coverage, minimum, mean and maximum dose of PTV and OAR, conformity index (CI), Nakamura CI (nCI), homogeneity index (HI), gradient index (GI), V12 Gy, time, number of beams, nodes and monitor units (MU). Values were normalized to the prescribed dose. Paired T-test was used to compare the parameters.

Results: The mean volume of the lesions were $19.5 \mathrm{~cm}^{3}$ (range $\left.2.3-56.8 \mathrm{~cm}^{3}\right)$. CI and nCI were significantly lower with IRIS plans $(p=0.048$ and $p=0.033)$, GI values were higher in MLC plans $(p<0.01)$. No significant patterns of conformity and gradient differences upon target shape (circular or irregular) and tumor volume (threshold of $10 \mathrm{~cm}^{3}$ ) were observed. PTV mean doses were higher with IRIS plans $(p=0.03)$. The number of MUs, beams and nodes were significantly lower with MLC plans resulting decreased treatment time $(p<0.01)$. No differences were observed in terms of coverage, HI, OARs and V12. Treatment characteristics and more detailed results will be presented during meeting.

Conclusion: Results of this study showed that Incise 2 MLC plans for brain metastases were dosimetrically feasible. MLC plans have steeper dose falloff, which could be an advantage for an OAR near the target. Treatment times of the MLC plans were approximately $30 \%$ lower which increase the delivery efficiency. Poster

\section{A dosimetric comparison of linac based deep inspiration breath-hold (DIBH) and tomotherapy treatment techniques of left-sided breast cancer}

F. Herberth, G. F. Fischer, K. Buchauer, L. Plasswilm and H. Schiefer

Klinik für Radio-Onkologie, Kantonsspital St.Gallen, St. Gallen, Switzerland

Aims: DIBH treatment techniques for the left-sided breast cancer are suitable to spare lung and heart dose. Due to its high dose modulation, Tomotherapy plans show generally a high plan quality. The goal of this study is to compare four different treatment techniques on the basis of multiple dose parameters.

Methods: 12 patients $(56.5 \pm 12.2$ yrs $)$ with breast carcinoma and adjuvant radiotherapy of the left side breast, including lymphatic pathways were retrospectively replanned using four planning techniques in each case: Breath-hold IMRT and VMAT irradiation techniques (IMRT-BH and VMAT-BH) for Varian TrueBeam STx Linacs were dosimetrically compared against free breathing techniques for Tomotherapy HD (both Tomo-direct and Tomo-helical).

Results: Even if a patient-to-patient comparison shows in all cases a lower mean dose to the heart for VMAT-BH, Tomo-helical leads to a comparable low value as VMAT-BH $(3.47 \pm 1.11$ Gy vs. $2.67 \pm 1.10$ Gy). VMAT-BH presents the best conformity index $(0.895 \pm 0.025)$ whereas Tomo-helical exhibits the highest dose homogeneity index $(1.071 \pm 0.023)$. Table 1 shows a qualitative evaluation (++: clearly favorable, ...,-: clearly unfavourable).

Conclusion: VMAT combined with a breath hold technique is a highly complex irradiation procedure, with excellent dosimetric outcome. Despite of the inferior separation between the heart and the irradiated region, Tomo-helical (with free breathing) leeds to a comparable reduction of the heart dose as for VMAT-BH, and remains therefore an equivalent alternative to VMAT-BH. Poster
Table 1 comparable dosimetric properties for Tomo-helical and VMAT-BH plans. In general, dynamic radiation techniques are superior to static techniques. But, each case requires an individual assessment

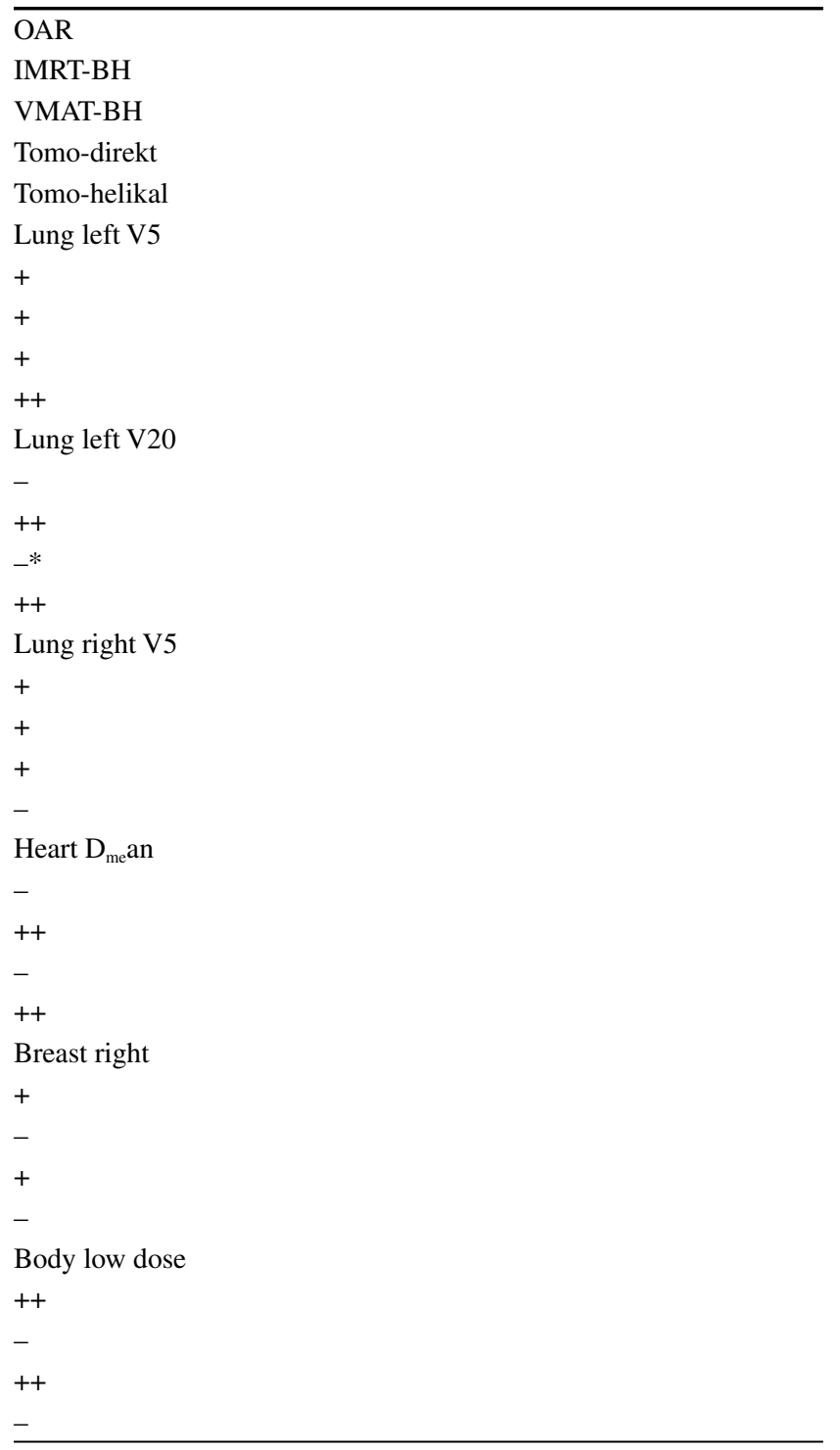

Real-time tracking deep inspiration breath hold for lung stereotactic body radiotherapy with electromagnetic transponders: First clinical experience

Dipasquale G. ${ }^{1}$, Jaccard M. ${ }^{1}$, Caparrotti F. ${ }^{1}$, Dubouloz A. ${ }^{1}$, Rakotomiaramanana B. ${ }^{1}$, Picardi, C. ${ }^{1}$, Plojoux J. ${ }^{2}$, Soccal P. M. ${ }^{2}$, Miralbell R. ${ }^{1}$

${ }^{1}$ Department of Radiation Oncology, Geneva University Hospital, Geneva, Switzerland

${ }^{2}$ Department of Pneumology, Geneva University Hospital, Geneva, Switzerland

Background: We report on the clinical implementation of stereotactic body radiotherapy of non-small cell lung cancer using deep inspiration breath hold (DIBH) controlled by real-time tumour tracking of implanted electromagnetic transponders (EMT).

Methods: Lung-specific EMT $(n=3)$ were implanted into bronchi adjacent to the tumour. A DIBH and a 4D-CT scan were acquired for 
planning. A dose of $60 \mathrm{~Gy}$ in eight fractions was prescribed to the planning target volume (PTV) and delivered with volumetric modulated arcs. Patient treatment set-up was performed in DIBH and with cone beam CT (CBCT) verification. Real-time tracking was undertaken by recording EMT motion and automatic interruptions of the beam-on mode for EMT deviation from the planned position above $3 \mathrm{~mm}$ in any direction was exceeded. Additional CBCT after, and $\mathrm{kV}$ during SBRT was also performed.

Results: The eight SBRT fractions were delivered as planned with good patient tolerance and no acute toxicity. The median [range] setup time per fraction was 11 [9.3-18.1] min and the treatment time was $1.2 \mathrm{~min}$. Visual inspection of CBCT before SBRT revealed perfect alignment of the PTV and organs at risk (OAR). The positions of EMT remained stable during DIBH treatments with median [range] absolute shifts of 0.14 [0.00-0.37] $\mathrm{mm}$. Automatic beam interruptions, triggered by detection of EMT positions out of tolerance due to patient coughing or releasing DIBH, occurred with a median of 5.5 times per fraction (range, 0-14). The DIBH planning succeeded to obtain better dosimetric results compared to using free breathing and an internal target volume created from the 4DCT.

Conclusion: Tracking with implanted EMT was reproducible and fast in DIBH, and allowed to reduce dose to the OAR. Posterwalk

\section{Lung feature tracking in 4D-MRI using a scale-invariant feature transform method}

\section{Emma Colvill}

Center for Proton Therapy, Paul Scherrer Institut, Villigen, Switzerland

Purpose: The purpose of this study is to demonstrate the feasibility of a scale invariant feature transform (SIFT) method for automated lung motion characterization from $2 \mathrm{D}$ navigator slices acquired during a 4D-MRI scan.

Methods: 4D-MRI scans of 20 healthy volunteers and 3 patients were acquired. The associated 2D navigator slices had a slice thickness of $6 \mathrm{~mm}$ and a frame rate of $3.33 \mathrm{~Hz}$. A SIFT-based method was employed to automatically extract features and corresponding trajectories tracked on the majority $(>75 \%)$ of frames with fewer than 20 misses. Misses and outlier data points were replaced by cubic interpolation. Outliers were identified as data points with a distance from the trajectory centroid greater than a threshold, defined as threshold $=P_{75}+1.5 \times P_{75-25}$ where $\mathrm{P}_{75}$ is the 75th percentile of the points-to-centroid distance distribution and $\mathrm{P}_{75-25}$ is the interquartile range (IQR). The locations of the features within the lung and motion trajectory information about each feature were calculated. Motion information included the mean range of the trajectory, IQR, and 5th and 95th percentiles.

Results: Preliminary results from five volunteer 4DMRI sets show that on average 5 features were tracked for each set, with the majority of features in the lower-to-middle portion of the lung with no features tracked in the apex of the lung. The average mean range, IQR and 5th and 95th percentiles of the feature trajectories were $4.8( \pm 2.7) \mathrm{mm}, 3.5$ $( \pm 2.0) \mathrm{mm}, 2.9( \pm 1.5) \mathrm{mm}$ and $3.3( \pm 1.6) \mathrm{mm}$ respectively, with inferior features displaying greater motion ranges than superior.

Conclusion: Automatic lung motion characterization based on 2D navigator slices was successfully demonstrated using a SIFT-based method. The features extracted from different portions of the lung displayed different motion characteristics. The method could be further improved by image pre-processing to allow for extraction of features in the apex. Oral presentation
Limitations of, and alternatives to, patient specific verification measurements for pencil beam scanning proton therapy

Michael Matter ${ }^{1,2}$, Lena Nenoff ${ }^{1,2}$, Gabriel Meier ${ }^{1}$, Damien Charles Weber ${ }^{1,3,4}$, Tony Lomax ${ }^{1,2}$, Francesca Albertini ${ }^{1}$

${ }^{1}$ Center for Proton Therapy, Paul Scherer Institut, Villigen,

Switzerland

${ }^{2}$ Department of Physics, ETH Zürich, ETH Hönggerberg, Zurich,

Switzerland

${ }^{3}$ Department of Radiation Oncology, University Hospital Zürich, Zurich, Switzerland

${ }^{4}$ Department of Radiation Oncology, University Hospital Bern, Bern, Switzerland

Aims: Patient specific verification (PSV) measurements for PBS proton therapy are resource-consuming and necessitate substantial beam time outside of clinical hours. As such, efforts for safely removing the PSV-bottleneck in the clinical work-flow are of great interest. Capabilities of current PSV methods to ensure the treatment integrity are investigated and compared to an alternative approach of reconstructing the dose directly from the machine control- or delivery log files with the help of an independent dose calculation (IDC).

Methods: Scenarios representing a wide range of delivery or workflow failures were identified (e.g. error in spot position, air gap or pre-absorber setting) and machine files altered accordingly. This yielded 21 "corrupted" treatment files, which were delivered and measured with our clinical PSV protocol. IDC machine- and log file checks were also conducted and their sensitivity at detecting the errors compared to the measurements.

Results: Although some of the failure scenarios induced clinically relevant dose deviations in the patient geometry, the PSV measurement protocol only detected 1 out of 21 error scenarios. However, 10 and all 21 scenarios were detected using dose reconstructions based on the log and machine files respectively.

Conclusion: Our data suggests that, although commonly used in particle therapy centers, PSV measurements do a poor job detecting data transfer failures and imperfect delivery machine performance. Machine- and log-file IDCs have been shown to represent a valuable, and more sensitive addition to the QA procedure, with the potential to replace PSV. Poster

\section{How can a limited number of proton fractions be optimally used in combined proton-photon treatments?}

\author{
S. Fabiano ${ }^{1}$, M. Bangert ${ }^{2}$, N. Andratschke ${ }^{1}$, M. Gubckenberger ${ }^{1}$, \\ J. Unkelbach \\ ${ }^{1}$ Department of Radiation Oncology, University Hospital Zurich, \\ Zurich, Switzerland \\ ${ }^{2}$ German Cancer Research Centre, Heidelberg, Germany
}

Aim: Currently, proton treatment slots are still a limited resource. To optimize proton slot allocation over an entire patient population, combined proton-photon treatments might play a role. Therefore, the aim of this study is to demonstrate how to optimally combine both modalities, also addressing the issue of the robustness of combined treatments against protons' range uncertainties.

Methods: Intensity-modulated radiation therapy (IMRT) and proton therapy (IMPT) plans were simultaneously optimized while accounting for fractionation effects through the biologically effective dose (BED) model. The method was investigated for different tumor sites (a spinal metastasis, one sacral chordoma, and an atypical meningioma) in which organs at risk (OARs) are located within or near the tumor. First, IMRT and IMPT plans were optimized based on the same set of objective and constraint functions. A reference plan was then generated as a simple proportional combination of the single-modality plans. Finally, a combined plan was optimized using the same planning ob- 
jectives. The probabilistic approach was applied to mitigate range uncertainties.

Results: In optimal combinations, proton and photon fractions deliver similar doses to OARs overlaying the target volume to protect these dose-limiting normal tissues through fractionation. Meanwhile, parts of the tumor are hypofractionated with protons. Therefore, the total dose delivered with photons is reduced leading to a reduction of the integral dose to normal tissues. The benefit of optimal combinations is also confirmed by the robustness analysis. For the spinal metastasis example, an optimal combination with 1 IMPT and 4 IMRT fractions achieves $37 \%$ of the mean BED reduction in normal tissues that a 5 -fraction IMPT plan yields, compared to $20 \%$ for the reference plan. Conclusion: Proton fractions are best used in combined treatments if parts of the tumor are hypofractionated with protons while near-uniform fractionation is maintained in serial OARs. Oral presentation

\section{Technician track}

\section{Post-operative radiotherapy and breath hold technique for right breastchest wall plus regional lymph-nodes single experience at IOS}

S. Leva ${ }^{1}$, M. I. Donegani ${ }^{1}$, D. Jevremovic ${ }^{1}$, M. Frapolli ${ }^{1}$, D. Daniele ${ }^{1}$, S. Moretto ${ }^{1}$, N. C. Azinwi ${ }^{1}$, D. Bosetti ${ }^{1}$, S. Cima ${ }^{1}$, I. Maddalena ${ }^{1}$, F. Martucci ${ }^{1}$, G.A. Pesce ${ }^{1}$, S. Presilla ${ }^{2}$, A. Richetti ${ }^{1}$, M. Tettamanti ${ }^{1}$, MC. Valli ${ }^{1}$

${ }^{1}$ Radiation Oncology Clinic, Oncology Institute of Southern Switzerland, Bellinzona, Switzerland

${ }^{2}$ Medical Physics Unit, Ente Ospedaliero Cantonale, Bellinzona, Switzerland

Aims: To evaluate the impact of deep inspiration breath hold (DIBH) in order to ensure organs at risk(OARs) sparing, when right breast/ chest wall including regional lymph-nodes radiotherapy (RT) is prescribed.

Methods: Six patients treated postoperatively on the breast (4 pts) or chest wall (2 pts) and ipsilateral IM/SC lymph-nodes were selected. The simulation CTs were made in free breathing (FB) and DIBH modalities. The treatments were delivered with VMAT/RapidArc technique in supine position with Posirest ${ }^{\mathrm{TM}}$. The dose prescribed was 50 Gy/2 Gy to whole breast or chest wall (PTV) plus $16 \mathrm{~Gy} / 2 \mathrm{~Gy}$ of boost (PTV BOOST) in case of conservative surgery. The treatments were planned in FB and DIBH modalities to compare the dose distribution to PTVs and OARs; both lungs, hearth, left anterior descending artery (LAD), contralateral breast. The constraints considered were: Dmax LAD 20 Gy; Dmean heart $<5$ Gy; V20 right lung:<35\%; Dmean both lungs: $<20 \mathrm{~Gy}$; Dmean left breast $<5$ Gy.

Results: The PTV coverage (95\%) varied from $95 \%-98 \%$ and $97 \%-$ 99\% in DIBH and FB respectively The Dmean heart varied between 2.2-4.9 Gy and 3.5-6.2 Gy; the LAD Dmax between 3-5 Gy and 3.58.5 Gy; the Dmean left breast between 2.8-5.0 Gy and 3.3-4.4 Gy; Dmean both lungs between 8.9-11.4 Gy and 9.2-12.1 Gy; the V20 right lung varied between $21 \%-32 \%$ and $21 \%-34 \%$ in DIBH and FB modalities respectively. The mean RT time was comparable in both modalities.

Conclusion: This experience reinforces our belief that DIBH should be applied even in right breast/chest wall RT to improve the dose reduction to hearth and LAD. A comparison between FB/DIBH planning modalities and different techniques is mandatory to choose the best RT treatment. Oral presentation
A new technique for robust volumetric modulated arc therapy treatment planning of craniospinal irradiation

Madalyne Chamberlain, Stephan Klöck, Claudia Linsenmeier, Mattia di Martino, Matthias Guckenberger and Stephanie Tanadini-Lang

Clinic for Radiation Oncology, University Hospital Zurich, Zurich, Switzerland

Objective: Craniospinal irradiation on a conventional linear accelerator most often has to be performed using multiple isocenters. This makes the junction regions prone to uncertainties against setup errors and patient motion. Often multiple plans with junctions at different locations are used to overcome this problem. Here we have developed a volumetric modulated arc therapy (VMAT) planning technique for craniospinal irradiation, which is robust against patient motion, and positioning inaccuracy between fields. Additionally the robustness of our technique was compared to a standard VMAT technique.

Method: A gradient dose technique has been developed, which is based on a $7 \mathrm{~cm}$ long perfect gradient dose created in Matlab. This gradient is used as a base dose plan for optimizing the brain and lower spine section of the plan. The middle section is created using the first plan as a base dose plan. This technique was tested on four patients previously treated in our clinic using standard VMAT technique. Each of these four patients were re-optimised using the gradient dose technique. The robustness of the plans were evaluated by shifting the upper isocenter closer to the second isocenter by $1 \mathrm{~mm}, 3 \mathrm{~mm}$, and $5 \mathrm{~mm}$, and comparing the maximum point doses with the original clinical plans.

Results: This new technique has been successfully implemented. Robustness was found to have improved using the gradient technique, as we observed a mean increase in maximum dose for the $3 \mathrm{~mm}$ shift of $3 \%$ (range $0 \%-6 \%$ ) for gradient technique and 17\% (range 11\%-28\%) for standard VMAT technique, respectively. This was even more pronounced for the $5 \mathrm{~mm}$ shift (4\% (range $0 \%-9 \%$ ) for gradient technique and $28 \%(11 \%-40 \%)$ for VMAT technique).

Conclusion: Our newly developed technique is significantly more robust against setup errors and patient motion for plans with multiple isocenters such as craniospinal irradiation. Poster

\section{Initial patient positioning without traditional skin marks:} Fiction or reality thanks to 3D surface- image-guidance?

Rubin $\mathrm{R}^{1}$, Bujanovic $\mathrm{N}^{1}$, Vetterli $\mathrm{D}^{1,2}$, Beer $\mathrm{K}^{1}$

${ }^{1}$ N/A, Radio-Oncology Center Biel, Biel, Switzerland ${ }^{2}$ Division of Medical Radiation Physics, Department of Radiation Oncology, Inselspital, Bern University Hospital and University of Bern, Bern, Switzerland

Aims: Initial patient positioning is traditionally performed with the help of skin marks. They are, however, problematic in many ways and therefore it is our aim to use 3D surface-image-guidance instead for daily initial patient positioning and to omit completely cumbersome skin marking. We will report on our clinical experience with initial patient positioning solely based on surface imaging and demonstrate the safe use of this positioning method in combination with final position correction using orthogonal $\mathrm{kV}-\mathrm{kV}$ imaging.

Methods: We retrospectively analyzed the accuracy of initial patient positioning based on skin marks ( 861 fractions, 39 patients) and based on surface imaging ( 834 fractions, 43 patients). Patients were positioned according to one of the above methods and treated to thoracic and abdominal body region and to breast after correction based on $\mathrm{kV}-\mathrm{kV}$ imaging, which served as gold standard. The mean of these $\mathrm{kV}$ based translational shift vectors and their standard deviations were determined and the results were compared between the two positioning methods.

Results: To achieve good results for surface imaging based positioning it is crucial to carefully select the region of interest for an optimal 
surface match. Regarding the entire group, no significant difference in the mean of the shift vectors between the two methods could be found. For breast treatments, the standard deviations were reduced slightly for surface imaging whereas for abdominal targets the opposite was the case for longitudinal direction.

Conclusion: With the necessary caution it is safe to omit skin marking for all patients and perform daily initial patient positioning with surface imaging only. This step is a major gain in patient comfort and it simplifies and speeds up the workflow. Poster

\section{Can surface-guided radiation therapy (SGRT) be used to eliminate tattoos and reduce setup time for SBRT lung treatments?}

J.Day, A.Moreira, B, König, N. Andratschke, M. Guckenberger, M. Zamburlini

Department of Radiation Oncology, University Hospital Zürich, Zurich, Switzerland

Objective: This study aimed at testing if it is possible to set up SBRT lung cancer patients without tattoos or skin marks, using only an SGRT device. Set up time using tattoos and skin marks versus an SGRT guide were also analyzed to compare efficiency.

Materials and Methods: 20 SBRT lung cancer patients received a $4 \mathrm{D}$ planning $\mathrm{CT}$ in the standard SBRT positioning at our center. 10 patients with a total of 37 fractions were set up and imaged using the current department standard. They were leveled with tattoos and then shifted to the isocentre. 10 of the patients with a total of 41 fractions were set up using an SGRT system. The patients were positioned in all 6 axes using the surface generated from the planning CT until all preshift deltas displayed on the SGRT systems were as close to zero as possible. After positioning all patients received a CBCT to verify patient position. This was online matched to the PTV in all 6 axes and all corrections were applied. For both groups set up time was measured as the time between when the plan was opened on the linac to the time of CBCT. The average shifts based on CBCT match of the PTV were also analyzed for both groups.

Results: Mean set up time was $7 \pm 2$ min and $10 \pm 3$ min in the SGRT group and tattoo group, respectively. Mean post-match shift based on CBCT was $0.25 \pm 0.24 \mathrm{~cm}, 0.34 \pm 0.24 \mathrm{~cm}, 0.14 \pm 0.13 \mathrm{~cm}$ in the SGRT group and $0.5 \pm 0.6 \mathrm{~cm}, 0.6 \pm 1.1 \mathrm{~cm}, 0.6 \pm 1.7 \mathrm{~cm}$ in the tattoo group, in the VRT, LNG and LAT directions respectively. Zero fractions from patients in the SGRT group had shifts larger than $1 \mathrm{~cm}$, compared to 12 fractions from 9 patients in the tattoo group.

Conclusion: Our study shows that SGRT alone provides a mean for more accurate and time efficient patient set up compared to tattoos or skin marks with the added benefit of increased patient comfort. Poster

\section{Can surface guided radiation therapy be used to setup DIBH breast cancer patients to reduce imaging?}

A.Moreira, M. Zamburlini, F. Cavelaars, M. Guckenberger, C. Linsenmeier, I. Pytko

Department of Radiation Oncology, University Hospital Zürich, Zurich, Switzerland

Objective: This study aimed at evaluating if the patient setup accuracy of surface guided radiation therapy (SGRT) for the treatment of left sided breast cancer patients in DIBH is enough to allow for a reduction of setup imaging for these patients.

Materials and Methods: 17 left sided breast cancer patients with a total of 296 fractions were treated using a combination of 3D tangential and IMRT fields in DIBH. The patients were positioned using an SGRT system (Align RT or OSMS) with first the free breathing CT reference surface to establish the vertical baseline followed by the DIBH $\mathrm{CT}$ reference surface. After positioning, online MV or KV imaging was performed in DIBH and matched on the chest wall. The patient position was corrected accordingly. Vertical (VRT), Lateral (LAT) and Longitudinal (LNG) shift values within $5 \mathrm{~mm}$ were considered within our tolerance.

Results: Out of the 296 fractions, differences between SGRT and IGRT were within our tolerance of $5 \mathrm{~mm}$ in all three directions in 277 fractions (93.6\%), and within $6 \mathrm{~mm}$ in 289 fractions (97.6\%). The largest difference of $16.6 \mathrm{~mm}$ was observed in lateral direction; however, the most frequent axis that was out of tolerance was longitudinal (13 out of 19 failed fractions). Mean post-match shift based on imaging was $-0.86 \pm 1.55 \mathrm{~mm}, 0.33 \pm 2.40 \mathrm{~mm}$, and $0.65 \pm 1.85 \mathrm{~mm}$ in the VRT, LNG and LAT directions respectively.

Conclusion: The data shows that SGRT is accurate within $5 \mathrm{~mm}$ in almost $94 \%$ of fractions for the setup of DIBH breast cancer patients. These results indicate a lack of systematic error as well as lack of influence of breast swelling on the accuracy of SGRT in DIBH breast patients. The combination of daily patient positioning using SGRT and setup imaging performed the first 3 fractions to establish patient stability would both reduce excess dose to the patient and treatment time on the Linear accelerator. Oral presentation

\section{0 years of $R T$, from Cobalt to HyperArc}

Rob Lodder

Radio-Onkologie, KSGR, Chur, Switzerland

My personal last 40 years of RT.

Developments in RT, Education, Machines, RT's,

31 foreign Chief-RT years in Switzerland.

15 s per year... Poster

\section{From empathic communication to therapeutic communication in radio-oncology}

Teacher Mamboury Nicolas, Lederer L., Trösch S., Zorneth M., Peroni M., Lucconi G., Bolsi A., Hrbacek J., Weber D.

Center for Proton Therapy, Paul Scherrer Institute, Villigen, Switzerland

Radiology, HESAV, Lausanne, Switzerland

The universe of radio-oncology has undergone a deep technological evolution over the last decade. The technical platforms, as well as the different treatment modalities have become more complex both in terms of imagery than irradiation techniques; this discipline has become very attractive, or even fun for young technicians in radio-Oncology.

In the face of this growing technology and medicine "household art style" in the words of some authors, so the risk incurred by the patient in this particular context is a reification, relegated to the rank of an object or simple Pathology to be irradiated.

The patient of a cancerous disease, weakened, rendered vulnerable, emotionally shaken and perhaps reached its dignity requires a personalized and empathetic support.

"The patient at the Center" is the leitmotif in the mouth of many health care providers. But that means you it really and how putting it into practice on the day the day, while the economy, effectiveness and efficiency are required?

The famous American psychologist Carl Rogers has laid the foundations for an approach centred on the person, empathy is an essential feature in the field of communication.

But a so-called empathetic communication can also be toxic or destructive depending on investment developed by one of the speakers or the used lyrics.

This communication proposes to a reflection on the modes of communication of the radiation oncology technician and the benefit to the patient.

Note: the epicene language is used to lighten the text. Poster 


\section{D vs. 3D positioning results for 4D treatments}

Lydia Lederer

Center for Proton Therapy, Paul Scherrer Institute, Villigen, Switzerland

Objective: In 2017, we have started to treat patients with moving targets at PSI. The workflow, specifically for positioning, is more complex than the one used for the treatment of static targets. In this report, we compare the corrections applied based on the 3D imaging vs. the ones resulting from 2D imaging, in an attempt to assess 3D positioning benefit.

Materials and Methods: This analysis was performed for 6 patients and 98 fractions. 4 patients presented were treated under anesthesia, 1 case was treated in the liver and 1 in the lung. Every day, 2 orthogonal topograms are performed and compared to the reference scouts (as for static cases) using reference points identified by the attending radiation oncologist. For 4D treatments, the positioning offsets applied to the Gantry 2 machine are based on the average CT (calculated from the 4D planning CT) and the daily average intensity projection low-dose CT. In this study, we compare the misalignments detected from the 2D imaging procedure (based on topograms) versus calculated with the $3 \mathrm{D}$ procedure.

Results: For $4(66.7 \%)$ of the 6 patients treated, the average difference (as well as the standard deviation) between 2D and 3D misplacements was below $2 \mathrm{~mm}$ along all directions, while highest values were obtained for the other 2 patients, one treated in the liver and the other in the lung, with differences in the average up to $4.9 \mathrm{~mm}$ lateral direction. Optimized results were found for children under anesthesia as compared to adults.

Conclusion: These data suggest that 3D imaging may not be necessary for all patients treated for moving targets with protons. Some localizations, however, do necessitate 3D imaging. Oral presentation

\section{First clinical evaluation of 4D ultrasound system for daily prostate IGRT: Comparison with cone beam CT}

M. Zahno, O. Matzinger, Z. Mazouni, A. Cosinschi, M. Pachoud, S. Ghandour

Interdisciplinary Department of Oncology, Riviera-Chablais Hospital, Vevey, Switzerland

Aims: Elekta Clarity ${ }^{\mathrm{TM}}$ 4D transperineal ultrasound autoscan system (TPUS) allows for real time prostate imaging during external beam radiation therapy. We prospectively studied the feasibility and accuracy of daily image guided radiotherapy (IGRT) using TPUS compared to concurrent routine cone beam CT (CBCT).

Methods: 10 localized prostate cancer patients were planned and treated with both CBCT and TPUS daily IGRT (390 treatment fractions). Every fraction, 4D ultrasound scan was performed first to account for prostate positioning. Suggested positioning corrections were recorded. Routine CBCT followed, subsequent positioning corrections were recorded and used for the actual treatment delivery. Acquisition time between TPUS and CBCT scans was kept to a minimum to avoid patient motion.

Results: The discrepancy in prostate positioning between the TPUS and CBCT was found to be $-0.8 \pm 1.8 \mathrm{~mm}$ laterally, $0.6 \pm 1.8 \mathrm{~mm}$ longitudinally and $0.1 \pm 3 \mathrm{~mm}$ vertically with no significant difference between the two modalities. Proper routine workflow integration is possible but is subject to a learning curve and necessitates extensive specific training.

Conclusion: Accuracy of prostate daily IGRT with TPUS is similar to CBCT. Routine use is feasible with proper training and could bring great radiation protection benefits to the patient. Poster

\section{When virtual reality meets radiotherapy}

S. Trigui, S. Rossi, F. Grozema, M. Barada, O. Nouveau, Dr T. Zilli, Dr. O. Fargier-Bochaton, Prof. R. Miralbell

Radio-oncology department, Geneva University Hospital (HUG), Geneva, Switzerland

Aims: A virtual reality project took place aiming to reassure patients enrolled in a radiotherapy treatment at the HUG. Moreover, this project allows us to form Radiation Therapy Technologist (RTT) and medical students with efficient tools.

Methods: The method used in the department is based on a virtual environment called "Virtual Environment of a Radiotherapy Treatment room "(VERT). This system simulates the treatment delivery by a linear accelerator in a 3D environment. A conference room is equipped with a 3D screen, a dedicated computer and remote controls such as in a real treatment room. Furthermore, VERT simulates the sound of the accelerator during treatment.

At this moment, there are two hospitals in Switzerland who have acquired this equipment among the hundred systems installed all over the world. Most of them are in the United Kingdom.

Results: For one year the HUG has been developing VERT to provide the most accurate patient and student experience. A total of 16 prostate cancer patients participated in viewing the virtual presentation. The VERT demonstration is prepared and exposed by RTT ideally before treatment.

In addition, over 25 RTT's and medical students have attended a VERT simulation.

Conclusion: Due to the positive participation, VERT presentations will be developed for different treatment regions e. g. breast cancer patients. Plans have been made to extend these presentations for the parents of young cancer patients. We noticed a real patient need to have additional information regarding their treatment. Moreover, questionnaires will be elaborated to have clear feedback of patients' experience and stress level before and after attending the virtual presentation. Poster

\section{Evaluating prostate EBRT treatment techniques and their robustness}

\section{Sabine Visser ${ }^{1}$, Carlos Calle ${ }^{1}$, Hans Neuenschwander ${ }^{1}$ \\ ${ }^{1}$ Radiation Oncology, Lindenhofspital, Bern, Switzerland}

Aim: A planning study for prostate cancer patients with no lymph node involvement was performed to evaluate planning techniques (3D-CRT, IMRT and VMAT) in terms of PTV coverage, OAR sparing and robustness.

Methods: Nine patients were selected retrospectively for this study. For each patient a $30 \times 2$ Gy 3 D-CRT plan, an IMRT plan and a dual-arc VMAT plan were created. In addition, the complexity of the PTV contours was analyzed by determining their concavity in the three cardinal projections. The PTV motion during treatment was simulated using Sun Nuclear's 3DVH/4D MotionSim platform, taking into account Calypso tracking data (20 patients, 780 fractions). A motion trajectory reflecting the corresponding histogram of deviations from the reference position (with $99 \%$ of all sampled data points exhibiting a deviation of less than $2.5 \mathrm{~mm}$ in all directions) was constructed and applied.

Results: VMAT had the best outcome in terms of conformity $(0.84 \pm 0.03)$ compared to IMRT $(0.73 \pm 0.03)$ and $3 \mathrm{D}-\mathrm{CRT}(0.76 \pm 0.05)$. Also, a more pronounced OAR sparing was achieved in case of VMAT, especially for the rectum. The rectum $\mathrm{V}_{50}$ was found to be $(31.3 \pm 12.4)$ $\%$ for 3D-CRT, $(23.3 \pm 8.2) \%$ for IMRT and $(18.1 \pm 5.9) \%$ for VMAT. The dose to the bladder was lowest for VMAT, too. It was found that the complexity of the most cranial axial PTV slice is significantly determining the OAR sparing capability of all the techniques. Visual inspection of the motion simulated DVH curves indicated a higher ro- 
bustness of IMRT vs. VMAT with respect to PTV motion. However, coverage of the respective CTV structures was not compromised.

Conclusions: For standard prostate cases, VMAT significantly reduces OAR doses compared to 3D-CRT and IMRT whilst maintaining or improving the coverage of the PTV. This result especially applies to more complex PTV shapes. A careful choice of CTV/PTV margins and/or the use of intra-fractional target volume monitoring/gating devices allow for a beneficial and safe application of VMAT prostate plans. Oral presentation

\section{Excess risk of second cancer induction from 3DCRT and VMAT breast radiotherapy}

A. Fogliata ${ }^{1}$, F. De Rose ${ }^{1}$, D. Franceschini ${ }^{1}$, A. Stravato ${ }^{1}$, M. Scorsetti ${ }^{1,2}$, L. Cozzi ${ }^{1,2}$

${ }^{1}$ Radiotherapy, Humanitas Research Hospital and Cancer Center, Milan, Italy

${ }^{2}$ Humanitas University, Biomedical Sciences, Milan, Italy

Aim: The use of VMAT in breast cancer radiotherapy increases the low-dose bath particularly in contralateral structures, relative to the classical 3DCRT. Aim of this work is the estimation of the excess absolute risk EAR in breast cancer radiotherapy using VMAT and 3DCRT. Methods: 20 patients with early stage breast cancer were selected for planning 40.05 Gy in 15 fractions. The VMAT arrangement had two partial arcs and a sector with no radiation delivery in front of the breast, with a total of $270 \pm 8$ active rotation degree; the 3DCRT plans setup had two tangents, for which two plans per patient were optimized: one as field-in-field (FinF) and one using hard wedges (3D_Wdg). Plans were calculated with Acuros in Varian Eclipse for accurate low dose levels estimation. VMAT optimization used the Photon Opimizer (vers. 13.6). Objectives minimized the dose in lungs, contralateral breast, heart and coronary artery. EAR was estimated using a model that uses carcinogenesis model and epidemiological data for carcinoma induction and accounts for cell repopulation/repair during the dose fractionation. EAR was computed for contralateral breast and lung. Normal tissue complication probability NTCP was computed for ipsilateral lung, heart and skin toxicity.

Results: EAR for contralateral breast carcinoma induction was estimated in 1.7, 2.4 and 2.8 [/10^4 patients per year] with FinF, VMAT and 3D_Wdg, respectively. For contralateral lung, the corresponding figures were $1.5,1.6$ and 2.1 [ $/ 10^{\wedge} 4$ patients per year]. NTCP, was significantly higher with 3DCRT relative to VMAT, with 3D_Wdg presenting the worst NTCP for all OARs.

Conclusion: VMAT, with proper arc setting, could have the same risk of second cancer induction as 3DCRT delivered with FinF, while reducing NTCP. VMAT might be considered a safe technique for breast cancer treatment for those aspects, while the use of hard wedges should be discouraged. Poster

\section{Dosimetric influences of different matching methods for breast treatments with soft tissue contour changes}

Füsers D., Roosenthaler S., Khan S., Ovcari D., Rabe E., Bodis S.

Radio-Onkologie-Zentrum KSA-KSB, Kantonsspital Aarau, Aarau, Switzerland

Aim: To investigate the influence of different matching methods on dose distribution in breast plans with a soft tissue contour change.

Method: Data from patients who were treated with tangential breast treatment technique and a soft tissue change $\geq 0,4 \mathrm{~cm}$ in the vertical direction was collected during this study. The soft tissue change was measured by using the mega volt (MV) electronic portal image device (EPID) images taken during patient set-up and compared with a digitally reconstructed radiograph (DRR). The selected patients have received a new planning CT (plCT). This
plCT was used for recalculation of the original plan. Dose volume histograms (DVHs) were calculated for three different matching methods (soft tissue, bone and compromise match) and compared to the DVHs of the original plan. The coverage of planning target volume (PTV) and dose to organs at risk (OARs) was compared. Additionally to the real patient data collection, a phantom study was performed to gather a reference data set and to prove the concept of the study.

Results: The data from seven patients have been collected so far. One of the patients had to be excluded from the study due to large rotational difference between the old and the new PLCT. The soft tissue match was suitable (based on PTV coverage and OAR dose) in $29 \%$ of the cases. The bone match was suitable in $43 \%$ of the cases and the compromise match between the both was suitable in $29 \%$ of the cases. In three cases, none of the match methods were suitable. The results of the comparison of the dose between the match methods and original PLCT, were not statistically significant for the lung $(P=0,85)$ and heart $(P=0,24)$, and for the PTV it was statistically significant $(P=0,04)$.

Conclusion: A bone match seems to be the optimal one for the PTV coverage and the dose to OARs. Poster

\section{Accuracy and reproducibility study for bladder volume measurement with a dedicated ultra sound bladder scan}

\section{A. Rebelo, F. Grozema, F. Francon}

Radio-oncology, Hôpitaux Universitaires de Genève (HUG), Geneva, Switzerland

Aims: Despite strict patient preparation instructions, reproducible bladder and rectum volume stays challenging. Ultra sound bladder volume measurements are non-invasive and a precise way of measuring pre-treatment bladder volume (PTBV) however, these are time consuming and costly. How accurate is a dedicated bladder ultra sound device in measuring the PTBV? Can PTBV by the use of a dedicated bladder scanner be introduced as standard pre-treatment volume verification, regarding time and accuracy?

Method: Prior to CT-simulation, bladder volume measurements using a dedicated bladder scanner have been performed for prostate patients, giving a reference bladder volume. This volume has been compared to the CT simulation bladder volume. Before treatment sessions, PTBV has been measured and compared with the daily CBCT, giving a daily volume difference. Measurement deviations caused by bladder filling have been minimalized by keeping the time between PTBV and CBCT to a strict minimum.

Results: In total 224 single measurements (18 patients) have been preformed, presenting a mean bladder volume difference of $29.46 \%$ between PTBV and CBCT with a mean standard deviation of $23.55 \%$. Single patient standard deviation (min. 5 measurements) has shown significant variations between $8.6 \%$ and $48.5 \%$. For $86.6 \%$ of daily comparison, CBCT bladder volume measurements showed an increase in bladder volume comparing to the preceding PTBV.

Conclusions: There is a significant accuracy variation between patients regarding the precision of the PTBV measurements, with a dedicated bladder scanner. Before introducing PTBV as standard pre-treatment verification, additional research is needed. Additional research will be performed to verify if these variations may be caused by anatomic variations, inconsistent user device manipulation or any other variability. The structural bladder volume increase may be caused by bladder filling after PTBV (during immobilization, $\mathrm{kV}$ imaging and CBCT acquisition). Poster 


\section{Comparing the precision of two rectal cancer treatment positioning and planning techniques: Prone positioning with 3DCRT plans and supine with VMAT plans}

\section{J. Gomes ${ }^{1}$, H. Härle ${ }^{1}$, Dr. U. Meier ${ }^{1}$}

Klinik für Radio-Onkologie, Kantonsspital Winterthur, Winterthur, Switzerland

Aims: To compare the stability of prone versus supine patient positioning for patients with rectal tumor T3-T4 treated with EBRT by analyzing setup deviations as well as to compare PTV and OAR irradiation between patients treated in the prone position with 3DCRT and in the supine position with VMAT plans. This will help determine which positioning and treatment planning technique is more precise and enables better OAR sparing and more complete PTV coverage. Methods: We collected the translational deviations of 13 patients in the supine position treated with VMAT technique, and 13 in the prone position treated with the 3DCRT technique, and calculated the systematic and random errors in each group. The doses and volume percentages corresponding to OAR and PTV were evaluated in the DVH for each patient. To compare the advantages of VMAT versus 3DCRT techniques directly, the DVH values of our patients treated in the supine position with VMAT were compared with patients treated in the supine position with 3DCRT technique found in literature. Results were calculated using the software SPSS version 22.0. Results: Supine positioning presented a lower magnitude of systematic and random errors and therefore higher precision as compared to prone positioning, however, there were no statistically significant differences. The prone positioning with BellyBoard and 3DCRT technique presents advantages in the decreased amount of small intestine, colon and bladder irradiated. With respect to the PTV, the supine position with VMAT achieved comparable dosing to prone positioning with 3DCRT. However, when comparing the planning techniques in just the supine position, VMAT results in lower doses to the OARs compared to 3DCRT. Conclusion: It is a sensible and plausible decision to change the prone position with 3DCRT to the supine position with VMAT. Posterwalk

\section{Evaluation of two cranial patient immobilization systems for stereotactic treatment}

Miklavcic A, Coppotelli M, Magdalenic J, Aspradakis M M, Jeller D, Götzfried T, Najafi Y, Zucchetti $P$

Institut für Radio-Onkologie, Luzerner Kantonsspital, Lucerne, Switzerland

Aims: Accurate and reproducible patient positioning in cranial stereotactic radiotherapy and radiosurgery (SRT/SRS) necessitates the use of immobilisation systems, so that any uncertainties in patient positioning and overall treatment are minimised.

In this work, inter- and intra-fraction errors from the use of two commercial systems were comparatively investigated: trUpoint ARCH and DSPS Double Shell Positioning System. Based on the patient positional accuracy achievable, appropriate margins were derived for the treatment planning system (TPS).

Methods: For this study, ten patients were immobilized with each system. In both cases, the mask system was outlined as part of the body for TPS. Treatment prescriptions were according to our institute's treatment protocols for SRT.

All patients were treated utilizing cone beam imaging (CBCT) for accurate patient positioning and volumetric modulated arc therapy technology on a Varian TrueBeamlinac. In order to investigate inter-fraction and intra-fraction set up uncertainties, image guidance during each treatment fraction involved the acquisition of three CBCT scans: one after initial patient setup on the treatment couch, one after the adjustment according to the six degrees of freedom registration of the CBCT dataset to the planning CT and the third one at the end of the treatment fraction.
The image registrations were performed through the automatic registration of the TrueBeam's X-ray Imaging system.

The resulting systematic and random errors were used to calculate the margin.

Results: The resulting margins accounting for residual and intra-fraction errors were $1.1 \mathrm{~mm}$ vertical, $0.6 \mathrm{~mm}$ longitudinal and $1.2 \mathrm{~mm}$ lateral for DSPS and for trUpoint ARCH $1.2 \mathrm{~mm}, 0.5 \mathrm{~mm}$ and $1.2 \mathrm{~mm}$, respectively.

Conclusion: Both immobilization systems provide comparable margins.

As a consequence of this evaluation, at our institution, a $1 \mathrm{~mm}$ minimum planning target volume margin is generally used for SRS. Posterwalk

\section{Twice versus thrice-weekly moderate hypofractionation for prostate cancer: Impact of overall treatment time}

Verane Achard ${ }^{1}$, Sandra Jorcano ${ }^{2}$, Michel Rouzaud ${ }^{1}$, Lluis Escudé2, Thomas Zilli ${ }^{1}$, Raymond Miralbell ${ }^{1}$

${ }^{1}$ Radiation Oncology, Geneva University Hospital, Geneva, Switzerland

${ }^{2}$ Radiation Oncology, Instituto Oncologico Teknon, Barcelona, Spain

Purpose: To evaluate the influence of overall treatment time (OTT) in disease control, acute, and long-term side effects with moderate hypofractionated external beam radiotherapy for prostate cancer delivered either in five $v s$ seven weeks.

Materials and Methods: From 2003 to 2017, 157 patients with localized prostate cancer (21 low-, 39 intermediate- and 97 high-risk, according to the NCCN classification) and an estimated risk of nodal metastases of $\leq 20 \%$ (Roach et al., 1994) were treated consecutively with 56 Gy in 4 Gy-fractions delivered either twice (87 patients, group 1, OTT $=45$ days $)$ or thrice ( 70 patients, group 2 , OTT $=30$ days $)$ a week using an IMRT $(85 \%)$ or VMAT $(15 \%)$ technique. Sixty-one patients (39\%) received, in addition, neoadjuvant and concomitant androgen deprivation (median duration of 6 months). Median age and PSA at diagnosis were 70 years (range, 48-86) and $7.9 \mathrm{ng} / \mathrm{ml}$ (range, 1.2-30), respectively. Gastrointestinal (GI) and genitourinary (GU) toxicities were scored according to the Common Terminology Criteria for Adverse Events version 3.0 grading scale. Biochemical relapse-free survival (bRFS) was assessed using the Phoenix definition. The median follow-up time was 110 and 56 months for group 1 and 2, respectively. Results: The 5-year bRFS was similar for both groups $(80.9 \pm 4.5 \%$ for group 1 and $85.0 \pm 4.9 \%$ for group $2, p=0.497$ ) as was the acute $\mathrm{GU}$ and GI toxicity. Five-year late urinary grade $\geq 2$ toxicity-free survival was higher in group $1(96.0 \% \pm 2.3 \%)$ than in group $2(81.2 \pm 4.9 \%$, $p=0.003$ ). No difference in 5-year grade $\geq 2$ late GI toxicity-free survivals was observed between both groups $(96.9 \pm 2.1 \%$ vs. $97.6 \pm 1.7 \%$ for group 1 and 2, respectively).

Conclusion: Against common radiobiological assumptions, longer OTTs may not be correlated with worse long-term outcome after curative radiotherapy for prostate cancer. However, more protracted OTTs may help to reduce moderate to severe late urinary toxicity-free survival. Poster 